

\section{GROUP IV ELEMENTS IN 2D STRUCTURES}

Michat Witold Ochapski 


\section{Composition of the graduation committee}

Chairman/secretary Prof. dr. J. N. Kok

Supervisor(s) Dr. ir. M. P. de Jong

Co-supervisor(s) Prof. dr. ir. H. J. W. Zandvliet

Committee Members Prof. dr. ir. G. Koster

Dr. ir. A. Y. Kovalgin

Prof. dr. T. Banerjee

Prof. dr. P. K. J. Wong

Dr. A. Fleurence

This work was financially supported by the Netherlands Organization for Scientific Research (NWO). Support is also acknowledged from the following institutes for having provided synchrotron beam time: ISA (Denmark), UVSOR (Japan), SOLEIL (France) and Photon Factory (Japan).

Printed by: Ipskamp

ISBN: 978-90-365-5025-3

DOI: 10.3990/1.9789036550253

(C) 2020 Michal Witold Ochapski, The Netherlands. All rights reserved. No parts of this thesis may be reproduced, stored in a retrieval system or transmitted in any form or by any means without permission of the author. Alle rechten voorbehouden. Niets uit deze uitgave mag worden vermenigvuldigd, in enige vorm of op enige wijze, zonder voorafgaande schriftelijke toestemming van de auteur. 


\title{
GROUP IV ELEMENTS IN 2D STRUCTURES
}

\author{
DISSERTATION
}

\author{
to obtain \\ the degree of doctor at the Universiteit Twente, \\ on the authority of the rector magnificus, \\ Prof.dr. T.T.M. Palstra, \\ on account of the decision of the Doctorate Board \\ to be publicly defended \\ on Wednesday 2 September 2020 at 14.45 hrs
}

by

Michał Witold Ochapski

born on 21 February 1990

in Turek, Poland 
This dissertation has been approved by:

supervisors

Prof. dr. M.P. de Jong (supervisor)

Prof. dr. H.J.W. Zandvliet (co - supervisor) 





\section{TABLE OF CONTENTS}

Introduction to 2D materials.......................................................................................... 1

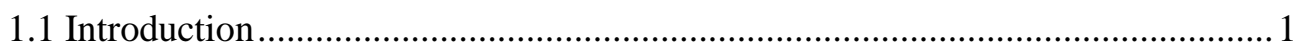

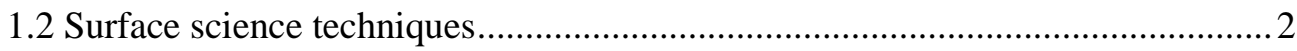

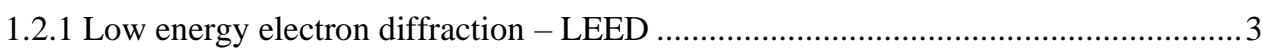

1.2.2 Scanning tunneling microscopy - STM........................................................... 6

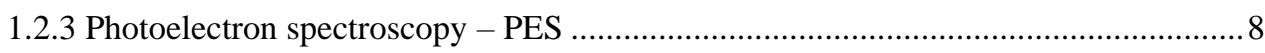

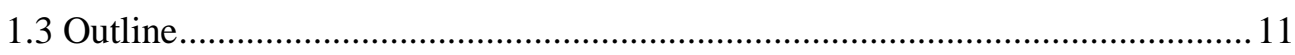

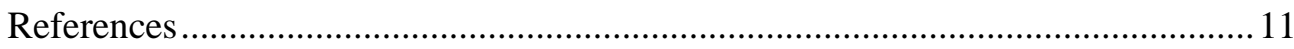

Substrates feasible for epitaxial growth of stanene...................................................15

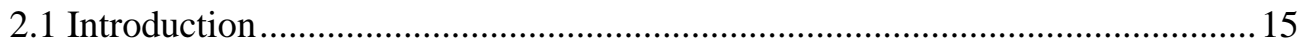

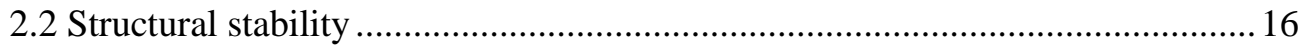

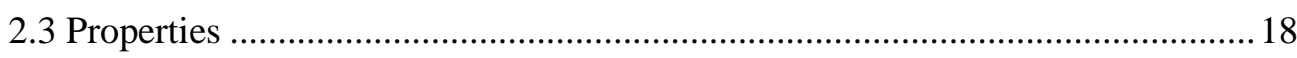

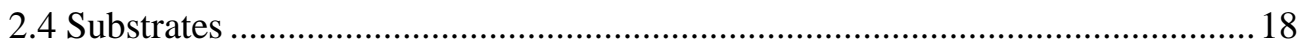

2.4.1 Substrates with reported realization of epitaxial of stanene...................................... 18

2.4.2 Substrates theoretically predicted to support epitaxial stanene..................................26

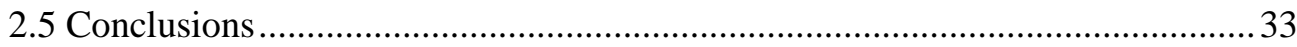

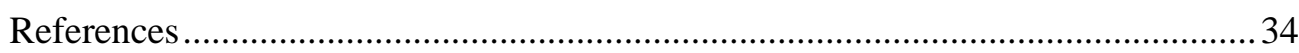

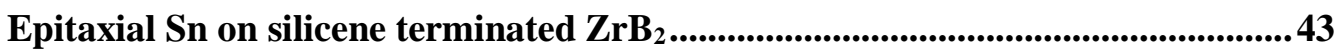

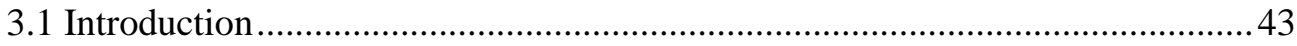

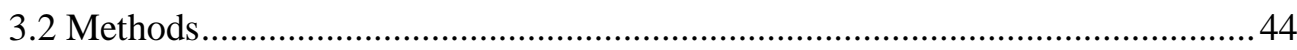

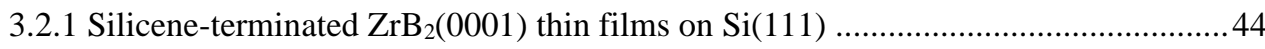

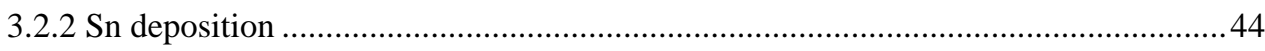

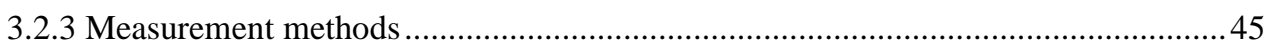

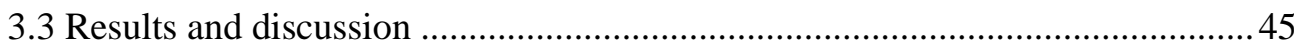

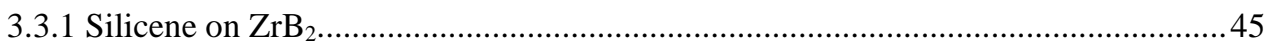

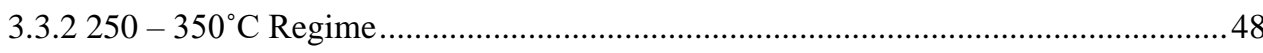




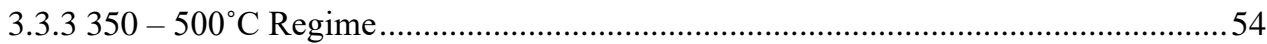

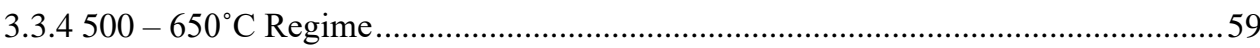

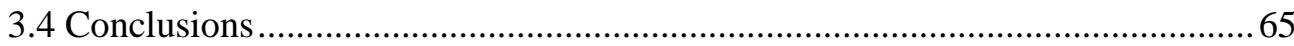

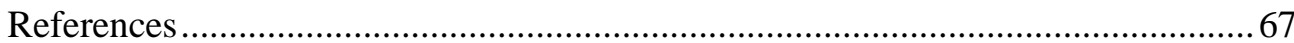

Epitaxial Sn on $\mathrm{MoS}_{2}$....................................................................................................... 75

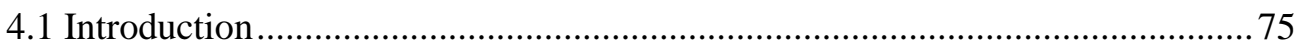

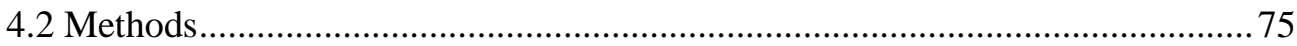

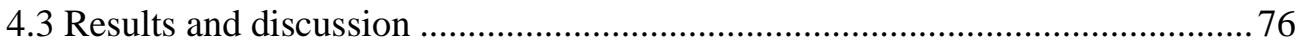

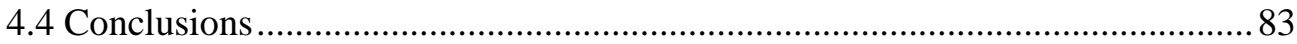

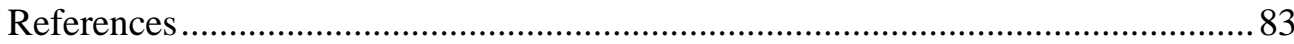

Li intercalation into multilayer graphene with controlled defect densities .........8 87

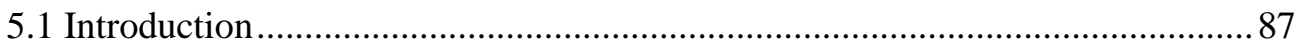

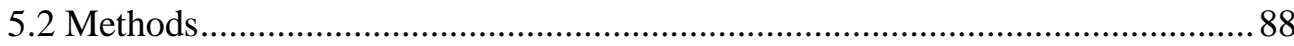

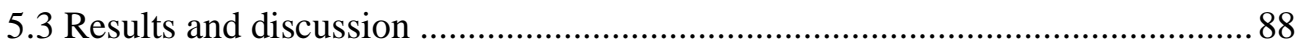

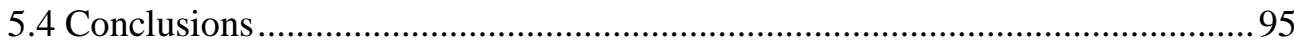

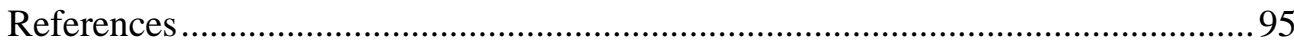

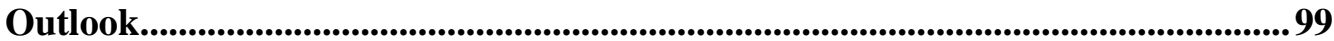

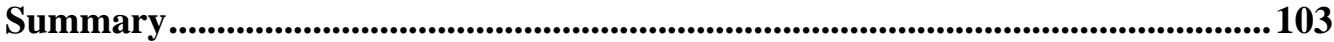

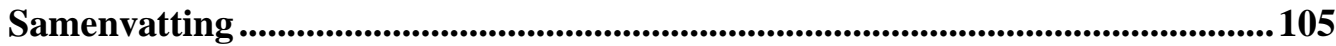

List of publications..................................................................................................................... 109

Acknowledgements.......................................................................................................................111 




\section{CHAPTER 1}

\section{Introduction to 2D materials}

\subsection{Introduction}

The discovery of graphene opened a new field in the physics of solid matter, focusing on two dimensional (2D) materials [1]. The newly obtained material turned out to have very unusual properties, the most interesting in its electronic aspect. Graphene shows linear dispersion around the Fermi level - a Dirac cone [2]. This makes the electrons velocity in graphene independent of energy, i.e. they behave as if they have no mass. It also exhibits very high carrier mobility [3]. Graphene came into existence at a similar time with the development of another new branch of (theoretical) solid-state physics, investigating topological states of matter [4]. What made the material really "famous" was that it is predicted to be a promising candidate for a variety of exotic topological properties [5]. From there, it has quickly grown to become one of the major research directions in today's field of materials science.

However promising, over 15 years of research on graphene has shown that some major obstacles on the way to utilizing its electronic properties are very hard to overcome. Even though theoretically possible, non-trivial topological effects are inaccessible for experimental physics, due to the extreme conditions in which those effects appear. The reason for this is the lack of a bandgap, which also hampers the application of graphene in semiconductor devices. The attempts of modifying graphene's electronic structure in order to open a significant bandgap with several techniques, like adsorbates, stress, external fields and even shape had only limited success. Hence, the attention of the community turned to other 2D materials, where this major problem would be easier to tackle. There are several directions including transition metal dichalcogenides and other 
layered Van der Waals materials, many of which are semiconductors with significant band gaps. However, these materials are not atomic monolayers in single layer form (see Fig. 1.1c), and their properties differ significantly from those of graphene. Nonetheless these materials are still interesting (for example due to the band gap dependence on the number of layers in some of them) and widely studied. Another reason is their feasibility of being stacked together in heterostructures with potentially new properties.

Another branch of the 2D materials field evolved from a graphene-inspired idea of elemental honeycomb atomic monolayers. Such materials would mimic graphene in its properties much more accurately, and many of them seem to be able to outshine graphene in the field of electronics. The main focus of this thesis is on such elemental 2D materials from Group IV - silicene, germanene, and in particular - stanene (see Fig. $1.1 \mathrm{~b})$. The properties of stanene are discussed in some detail in chapter 2 .

a)

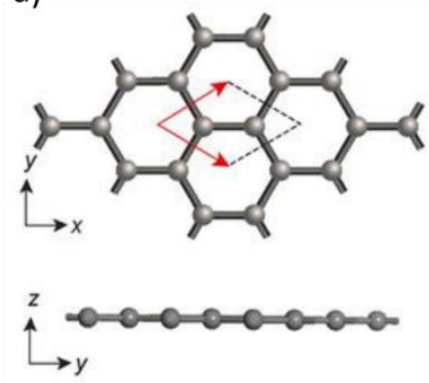

b)

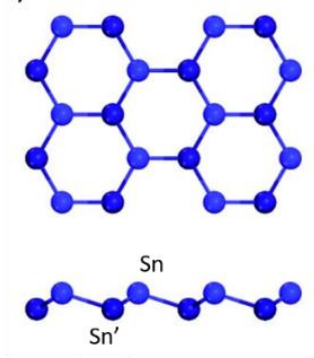

c)
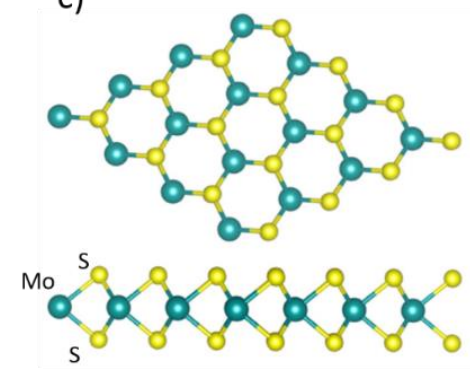

Fig. 1.1 Atomic models of various 2D materials. a) Graphene is an elemental 2D material with an atomically flat honeycomb lattice. Reproduced from [6]. b) The lattice of stanene as well as several other elemental 2D materials exhibit a degree of buckling [7]. c) $\mathrm{MoS}_{2}$ is a Van der Waals layered material with (when viewed from the top) honeycomb lattice that consist of three covalently bonded atomic sheets (S, Mo, S). Reproduced from [8].

\subsection{Surface science techniques}

Due to their atomically thin structure, 2D materials are ideally suited for probing with surface science techniques that allow for experimental characterization of the surface properties of materials. Many of such techniques require high cleanness of the surface and are sensitive to contamination due to the nature of 2D materials. While graphene itself is quite inert to ambient conditions, others, and in particular materials discussed in this thesis, are by themselves very sensitive to contamination and possible 
degradation or decomposition due to atmospheric pressure and contact with more reactive elements. To avoid these problems, surface science techniques are performed in ultra-high vacuum (UHV) conditions, with pressure of the order of $10^{-10}$ mbar. Many 2D materials are also prepared in UHV and then measured in-situ. In this work, all the experimental process, comprising sample preparation and measurement were performed in-situ. The sample preparation techniques were thermal evaporation and annealing. Evaporation is a physical vapor deposition (PVD) technique. In thermal evaporation, the material to be deposited is put into a (ceramic or an inert metal) crucible which is radiatively heated, until the material evaporates. The sample is then kept in the vicinity of the source, so the evaporating material can adsorb on the sample surface. Annealing is a technique used to increase the temperature of the sample in order to increase the degree of structural order, achieve a phase transition, or stimulate some chemical reaction. In this work, several annealing techniques were used - radiative, e-beam and direct current heating. Radiative annealing is the simplest one, where a filament is heated up by direct current and put in close vicinity of the sample. In e-beam annealing, the sample is heated up by electron bombardment from a hot filament at high voltage (either the sample or the filament can be at high voltage). This requires conducting samples, and should be done such that the electrons bombard the backside of the sample to avoid damage. Finally, DC heating heats the sample by passing a DC current through it. For this method to be effective, the samples need to be quite resistive (otherwise extremely high currents are required). It works well for (doped) Si wafers, which are commonly used substrates in the field. As for measurement techniques, we utilized several complementary surface science techniques which allow for detailed characterization of structural and electronic aspects of the studied samples.

\subsubsection{Low energy electron diffraction - LEED}

Low energy electron diffraction is an experimental technique used for determination of the structural properties such as size, symmetry and rotational alignment of unit cells present on the surface. In particular, it is often used to study surface lattices of adsorbates. Fig. 1.2a shows a schematic illustration of a LEED system. In ultra-high Vacuum (UHV), the electrons emitted from a hot filament are accelerated to a predefined energy onto the sample surface by an electron gun. The interference of the incident electrons scattered on the crystal lattice of the sample results in a diffraction pattern, which can be observed on a fluorescent screen. Several grids are typically located between the screen and the sample, to filter the inelastically scattered electrons. 
LEED is a very useful and fast tool to examine if a deposited material formed an ordered structure on the surface of a substrate.

The principle behind LEED utilizes the wave-like nature of electrons. Electrons, according to the de Broglie relation, have a wavelength $\lambda_{e}$ of:

$$
\lambda_{e}=h / p=h / \sqrt{2 m V e},
$$

where $h$ is the Planck constant and $p$ is the electron momentum, $m$ is the electron mass, $e$ is the electron charge, and $V$ is the acceleration voltage. This equation gives a relation between an applied voltage and the electron wavelength. Based on this concept there are two fundamental requirements that $\lambda_{e}$ needs to fulfill to be useful in LEED technique: (i) it needs to be small enough to be diffracted by an atomic lattice and (ii) the energy of the electrons needs to be such that the inelastic mean free path $\lambda_{I M F P}$ of the electron is minimized. $\lambda_{I M F P}$, in simple words, is the average distance which an electron can travel through a material before it is scattered inelastically (see Fig. 1.2c). These two criteria are fulfilled when the energy of the electrons is between 20 and $400 \mathrm{eV}$. In this range, the electron diffraction pattern can be observed and the penetration depth is low enough to probe only the outermost atomic layers.

The incident electrons are emitted from the electron gun by a cathode filament and focused by a set of electrostatic lenses. Upon reaching the surface the electrons scatter elastically from the atomic lattice of the sample. The diffracted electron waves that interfere constructively obey the Bragg equation, which is of the form (see Fig. 1.2b)

$$
n \lambda_{e}=d \sin \varphi,
$$

where $n$ is an arbitrary integer, $\lambda_{e}$ is the electron wavelength, $d$ is interatomic distance in the lattice of the sample and $\varphi$ is the angle between the incident and scattered electron "ray". The interaction between scattered electrons and atomic lattice is most conveniently described in terms of reciprocal space. For an incident wave number $k_{\boldsymbol{\theta}}=$ $2 \pi / \lambda_{0}$ and the scattered one $k=2 \pi / \lambda$, the condition for constructive interference is given by the Laue condition, which is simply a reciprocal space equivalent of the Bragg equation. This representation is readily observed on a so-called Ewald sphere (see Fig. 
1.2d). The diffraction pattern observed on the fluorescent screen is a direct image of the reciprocal lattice of the surface.
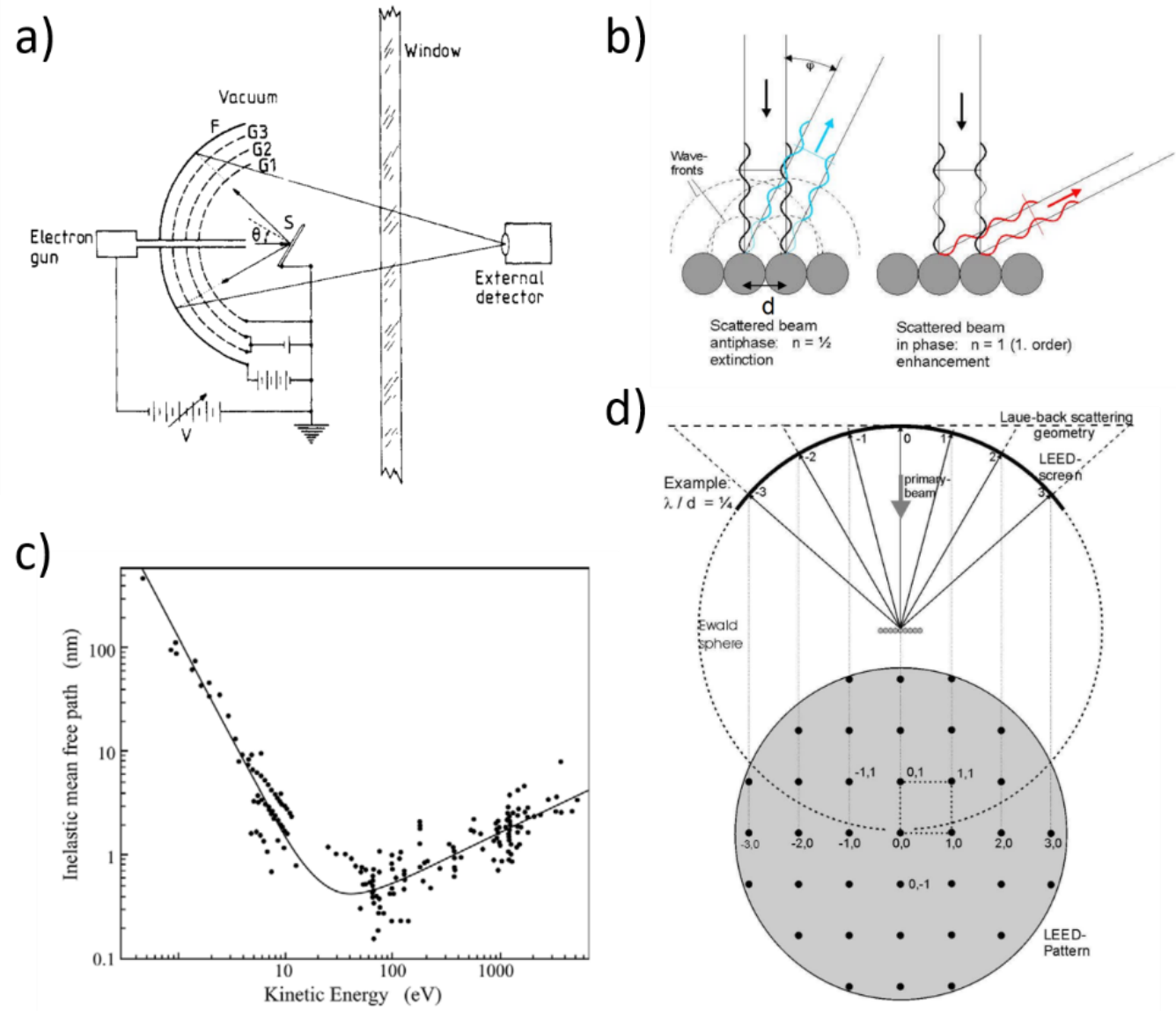

Fig 1.2: a) Diagram of a LEED system. Reproduced from [9]. b) Electron diffraction process. Reproduced from [10] c) Inelastic mean free path universal curve. Note that the electrons within $20-200 \mathrm{eV}$ range have $\lambda_{I M F P}<1 \mathrm{~nm}$. Reproduced from [11] [12]. d) Exemplary diffraction pattern and corresponding Ewald sphere. Reproduced from [13] 


\subsubsection{Scanning tunneling microscopy - STM}

Scanning tunneling microscopy (STM) is an experimental technique to characterize the topography of the sample surface. The experimental setup consists of a metal tip (the most common are $\mathrm{Pt} / \mathrm{Ir}$ and $\mathrm{W}$ tips) that is put in nanoscopic vicinity of the sample usually about $1 \mathrm{~nm}$ away. When a bias voltage is applied between the sample and the tip, a tunneling current can be detected. In a one-dimensional tunneling problem this current is given by: [14]

$$
I \propto e^{-2 \kappa z},
$$

where $I$ is the tunneling current, $z$ is the tip-sample distance and $\kappa$ is the decay constant for the wave function in a potential barrier. Therefore, such current depends exponentially on the barrier width, which corresponds to the tip-sample distance $z$. Only conducting and semiconducting samples are feasible for being studied by this method, since the tunneling current needs to be detected and controlled. The tip is mounted to a piezo-element that allows the tip to move in 3 directions with respect to the sample surface. An electronic feedback loop keeps the tunneling current constant, by varying $z$. In that way, in the so-called constant current mode, the tip can scan the surface in the $x$ $y$ plane and image the "topography" of the sample (by plotting the variation of $z$ ). One should keep in mind however, that since an electronic current is used to scan, what is really measured is also strongly dependent on the local density of states (DOS). The STM signal is therefore a mixture of electronic and topographic components. This method enables measurements with atomic resolution, making it possible to detect individual atoms.

Another way to utilize the such system is scanning tunneling spectroscopy (STS). The technique takes advantage of the fact, that in stable temperature conditions and with featureless density of states of the tip, the local density of states of the sample $\rho_{S}$ is proportional to the deritavite of the tunneling current over the bias voltage:

$$
\frac{d I}{d V} \sim \rho_{S}
$$

This technique allows for the measurement of local density of states on the sample, and in its more advanced version to make $d I / d V$ maps of the sample surface. 
a)

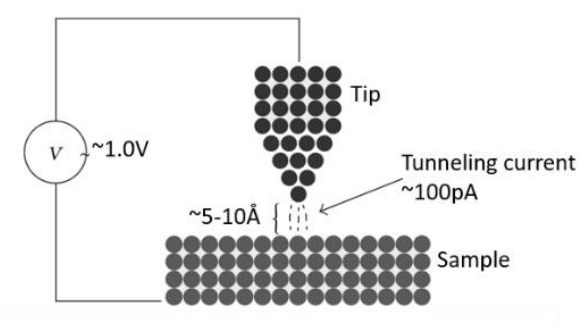

c) b)

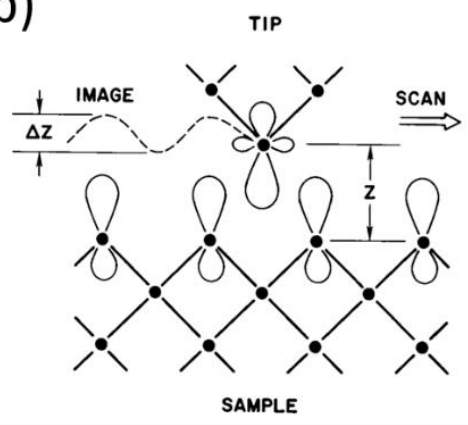

sample - tip sample +

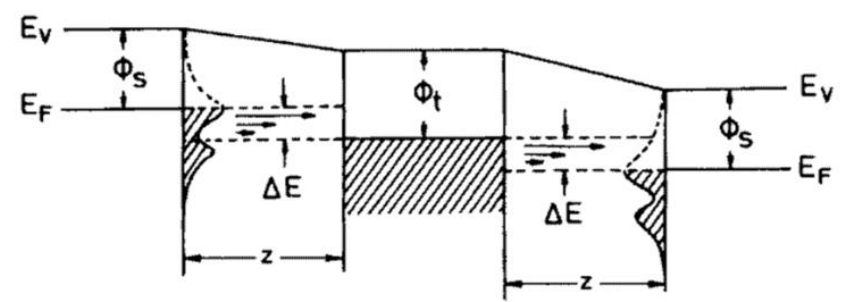

Fig. 1.3: a) Schematic illustration of the STM system with typical orders of magnitude of bias voltage, tip-sample distance and tunneling current. Dark spheres represent atoms in the sample/tip. Adapted from [15]. b) Microscopic view of STM imaging mechanism. The tunneling conductance is generated by the overlap of the tip state with the atomic-like states on the sample. Reproduced from [17]. c) Schematic energy diagram for the tunnel junction between a metallic tip and an adsorbate-covered metal surface. The left (right) junction corresponds to a negative (positive) bias on the sample to permit tunneling from occupied states of the sample (tip) into empty states on the tip (sample). Only states within the energy window $\Delta E$ can contribute. $\varphi_{s}$ and $\varphi_{t}$ are the local work functions (barrier heights) for the sample and tip, respectively, whereas $z$ is the tunnel gap width. Reproduced from [16]. 


\subsubsection{Photoelectron spectroscopy - PES}

Photoelectron spectroscopy is a technique that gives insight into the chemical composition and electronic (band) structure of the sample surface. The technique allows to identify elements present on the surface, as well as gives information about chemical bonding. It also allows for estimation of layer thickness for ultrathin films, provided that the sample composition is well known. PES is based on the famous photoelectric effect, discovered by Hertz and explained by Einstein. By absorbing photons with energy $h v$, electrons can gain enough energy to escape the potential well of the system to which they are bound (a metallic solid in the examples below). This can be done at the cost of overcoming the barrier formed by the electron energy level with respect to the Fermi energy - the binding energy $E_{B}$ - and the work function of the surface $\phi$, according to the equation:

$$
E_{k}=h v-E_{B}-\phi .
$$

The remaining energy is the kinetic energy $E_{K}$ of the photoelectron. In PES, significant interest is put on the core levels - energy levels far below the Fermi energy. Due to the fact that each element has specific binding energies of its core levels, PES is an excellent tool for chemical characterization of the sample (the technique was originally coined "Electron Spectroscopy for Chemical Analysis", in short ESCA, by its inventor Siegbahn). The signal comes from only the outermost layers, which is connected to the aforementioned electron inelastic mean free path $-\lambda_{\text {IMFP }}$ (see Fig. 1.2c), which can be varied with the photon energy $\left(\lambda_{I M F P}\right.$ depends on $\left.E_{K}\right)$. The obtained signal intensity is proportional to the number of emitted electrons vs binding energy. At binding energies corresponding to the core levels of elements present on the surface there will be peaks in intensity. The intensity, position and shape of the peaks give insight, among others, into the abundance, chemical environment, and the degree of disorder in the chemical environment of a given element in the sample.

The PES system consists of a photon source, and a detector (see Fig. 1.4a). In lab-based systems the photon source typically emits $\mathrm{X}$ - rays (hence the method is often called $\mathrm{X}$ - ray photoelectron spectroscopy - XPS), generated by bombarding a metallic anode with high-energy electrons. Usually only a few pre-defined photon energies are available. To overcome that limitation, as well as to achieve higher energy resolution, synchrotron radiation is used for high-quality PES measurements. This allows for a much broader range of well controlled photon energies. Fig. 1.4a illustrates 
schematically the geometry of the PES system. The incident photons get absorbed by the core level electrons, which escape the sample and then are accelerated to the detector, which measures the intensity (number of detected electrons in a certain time interval) at each kinetic energy.

a)

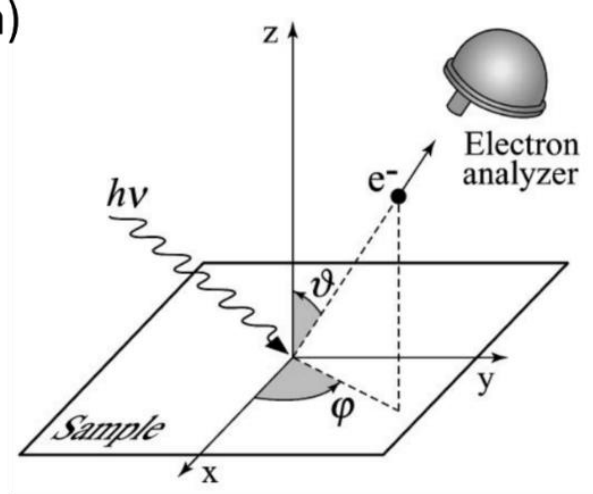

c)

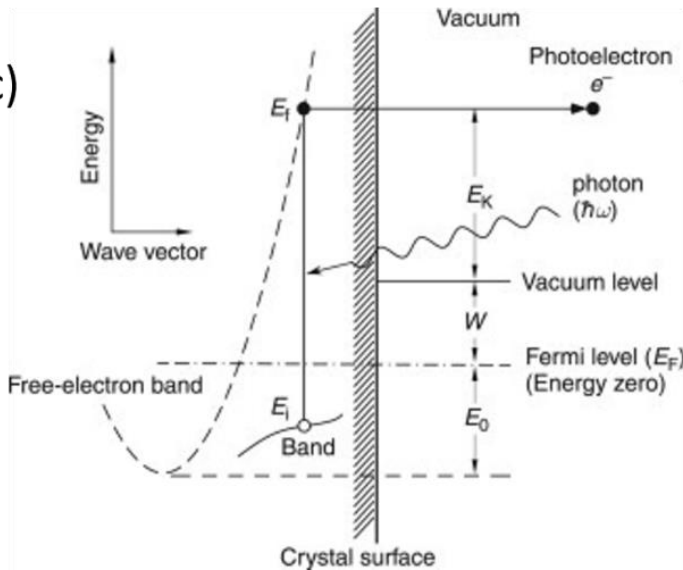

b)
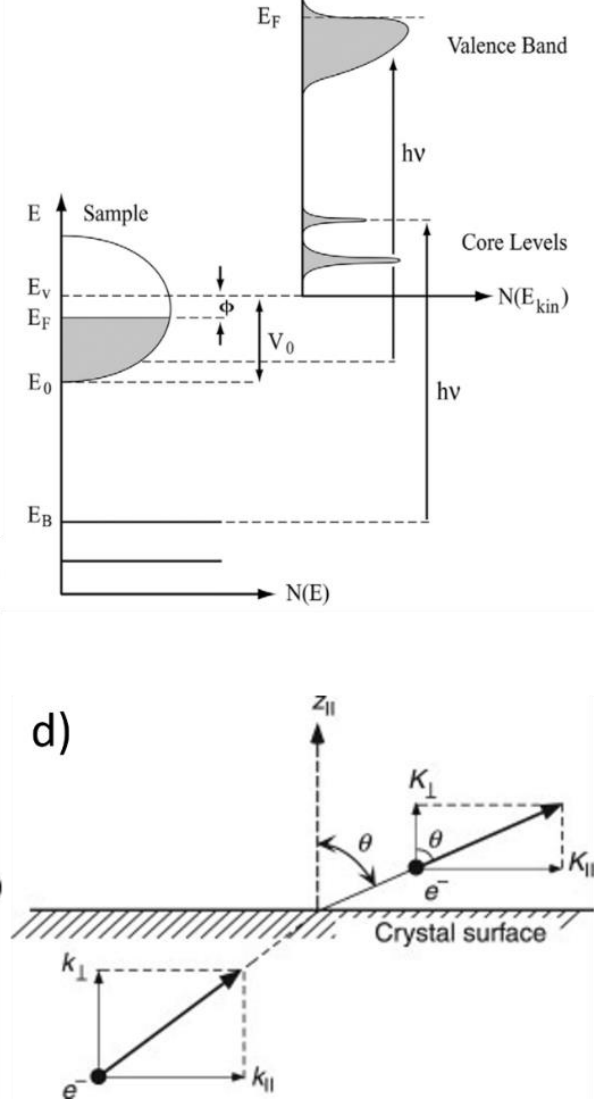

Fig. 1.4: a) (AR)PES experimental set up, the polar and azimuthal directions are specified by the angles $\vartheta$ and $\varphi$, respectively. b) The photoelectron energy distribution (right), with respect to the energy levels in a solid (left). Reproduced from [18] [11] . c) Energy-conservation diagram of the photoemission process. d) Momentum parallel to the surface of photoelectrons is conserved during the photoemission process. Reproduced from [19]. 
Angle-resolved photoelectron spectroscopy (ARPES) is a more sophisticated technique based on the same principle as PES. The experimental system for ARPES is identical to that used in PES, except the detector collects additionally the information about the exit angle $(\vartheta$ in Fig. 1.4a) of the electrons. This allows to image a band structure of the sample surface.

In addition to the photoelectric effect, ARPES makes use of two very basic concepts the energy of the photon - electron system should be conserved and so should be the momentum. Imagine an electron in an initial state $E_{i}$ [19]. By absorbing a photon with the energy $\hbar \omega$ it is excited to the state at the energy $E_{f}$. From energy conservation we have:

$$
E_{f}=\hbar \omega+E_{i} .
$$

In the case of photoelectron, the excited state is a free-electron state in vacuum, for which the energy is expressed by

$$
E_{f}=E_{k}+W
$$

where $E_{k}$ is the kinetic energy and $W$ - the work function of the sample. The parallel component of the momentum is conserved in the whole process (the photon has negligible momentum), due to translational symmetry of both the crystal structure in the surface plane as well the vacuum. The parallel component of the photoelectron momentum can be expressed by the formula:

$$
k_{\|}=\frac{\sqrt{2 m E_{k}}}{\hbar} \sin \vartheta=\frac{\sqrt{2 m\left(E_{i}+\hbar \omega-W\right)}}{\hbar} \sin \vartheta,
$$

where, $k_{\|}$is the in-plane component of the momentum $k, \vartheta$ is the angle between the direction of the momentum $k$ and a vector normal to the sample surface plane (Fig. 1.4a). This relation allows for obtaining a slice of the energy dispersion relation $E_{k}\left(k_{\|}\right)$ in the $\boldsymbol{k}_{\|}=\boldsymbol{k}_{x}+\boldsymbol{k}_{y}$ direction in the sample. In practical applications, only the high symmetry directions of $\boldsymbol{k}_{\|}$in the Brillouin zone are investigated. There is also a possibility for imaging the whole Fermi surface, by recording slices at different angles $\vartheta$ and $\varphi$. Some modern systems, with a (near) $180^{\circ}$ acceptance angle for emitted photoelectrons, are able to perform such a measurement without having to rotate the sample at all. 


\subsection{Outline}

This thesis is a contribution to the $2 \mathrm{D}$ materials field, with a particular focus on group IV elemental 2D materials. By utilizing several complementary surface science techniques we investigated the formation of ordered structures in 2D regime and interactions between these materials and various substrates. In chapter 2, we review and systematize the experimental aspects of the knowledge about substrates suitable for epitaxial growth of stanene. Chapters 4 and 5 presents our work on growth of epitaxial Sn layers on different substrates - silicene terminated $\mathrm{ZrB}_{2}$ (chapter 4) and $\mathrm{MoS}_{2}$ (chapter 5). Finally, Chapter 5 shows an investigation on $\mathrm{Li}$ intercalation in between graphene sheets, focusing on how defects may influence the intercalation capacity of multilayer graphene.

\section{References}

[1] K. S. Novoselov, "Electric Field Effect in Atomically Thin Carbon Films," Science, vol. 306, no. 5696, pp. 666-669, Oct. 2004, doi: 10.1126/science.1102896.

[2] P. R. Wallace, “The Band Theory of Graphite," Phys. Rev., vol. 71, no. 9, pp. 622-634, May 1947, doi: 10.1103/PhysRev.71.622.

[3] K. I. Bolotin et al., "Ultrahigh electron mobility in suspended graphene," Solid State Commun., vol. 146, no. 9-10, pp. 351-355, Jun. 2008, doi: 10.1016/j.ssc.2008.02.024.

[4] M. Z. Hasan and C. L. Kane, "Colloquium: Topological insulators," Rev. Mod. Phys., vol. 82, no. 4, pp. 3045-3067, Nov. 2010, doi: 10.1103/RevModPhys.82.3045.

[5] C. L. Kane and E. J. Mele, "Quantum Spin Hall Effect in Graphene," Phys. Rev. Lett., vol. 95, no. 22, p. 226801, Nov. 2005, doi:

10.1103/PhysRevLett.95.226801.

[6] R. Huang, "Theoretical study in carrier mobility of two-dimensional materials," IOP Conf. Ser. Mater. Sci. Eng., vol. 231, p. 012116, Sep. 2017, doi: 10.1088/1757-899X/231/1/012116. 
[7] Y. Xu et al., "Large-Gap Quantum Spin Hall Insulators in Tin Films," Phys. Rev. Lett., vol. 111, no. 13, p. 136804, Sep. 2013, doi:

10.1103/PhysRevLett.111.136804.

[8] Q. Wang, P. Wu, G. Cao, and M. Huang, "First-principles study of the structural and electronic properties of $\mathrm{MoS}_{2}-\mathrm{WS}_{2}$ and $\mathrm{MoS}_{2}-\mathrm{MoTe}_{2}$ monolayer heterostructures," J. Phys. Appl. Phys., vol. 46, no. 50, p. 505308, Dec. 2013, doi: 10.1088/0022-3727/46/50/505308.

[9] F. Jonat and J. A. S. Jr, "Low-energy electron diffraction for surface structure analysis," p. 60.

[10] G. Ertl and J. Küppers, Low Energy Electrons and Surface Chemistry, 2nd ed. Weinheim: VCH Verlagsgesellschaft mbH, 1985.

[11] A. Damascelli, "Probing the Electronic Structure of Complex Systems by ARPES," Phys. Scr., vol. T109, p. 61, 2004, doi: 10.1238/Physica.Topical.109a00061.

[12] M. P. Seah and W. A. Dench, "Quantitative electron spectroscopy of surfaces: A standard data base for electron inelastic mean free paths in solids," Surf. Interface Anal., vol. 1, no. 1, pp. 2-11, Feb. 1979, doi: 10.1002/sia.740010103.

[13] M. Henzler and W Gopel, Oberflachenphysik des Festkorpers, 2nd ed. Stuttgart: B. G. Teubner, 1994.

[14] P. K. Hansma and J. Tersoff, "Scanning tunneling microscopy," p. 24, 2014.

[15] H. Yaghoubi, “The Most Important Maglev Applications,” J. Eng., vol. 2013, pp. 1-19, 2013, doi: 10.1155/2013/537986.

[16] F. Besenbacher, "Scanning tunnelling microscopy studies of metal surfaces," Rep. Prog. Phys., vol. 59, no. 12, pp. 1737-1802, Dec. 1996, doi: 10.1088/00344885/59/12/004.

[17] C. J. Chen, "Microscopic view of scanning tunneling microscopy," J. Vac. Sci. Technol. Vac. Surf. Films, vol. 9, no. 1, pp. 44-50, Jan. 1991, doi: 10.1116/1.577128.

[18] A. Damascelli, Z. Hussain, and Z.-X. Shen, "Angle-resolved photoemission studies of the cuprate superconductors," Rev. Mod. Phys., vol. 75, no. 2, pp. 473-541, Apr. 2003, doi: 10.1103/RevModPhys.75.473. 
[19] T. Sato and T. Takahashi, "Angle-Resolved Photoemission Spectroscopy of Graphene, Graphite, and Related Compounds," in Comprehensive Semiconductor Science and Technology, Elsevier, 2011, pp. 383-409. 



\section{CHAPTER 2}

\section{Substrates feasible for epitaxial growth of stanene}

\subsection{Introduction}

After the discovery of graphene, a two-dimensional (2D) material (single layer of C atoms) with exceptional electronic properties, interest in other 2D materials has skyrocketed. One of the most attractive properties of graphene is that its charge carriers behave as massless Dirac fermions,[1] which leads to exceptionally high mobility values on the order of $10^{5} \mathrm{~cm}^{2} \mathrm{~V}^{-1} \mathrm{~s}^{-1}$.[2] A two-dimensional (2D) topological insulator phase, the quantum spin hall insulator (QSHI), has also been predicted to exist in graphene [3]. The spin-orbit coupling (SOC) that lies at the origin of this state is very weak though, such that the effects may only be observed at extremely low temperatures [4]. Other elements of group IV, e.g. Si, Ge and $\mathrm{Sn}$, exhibit the same valence electronic configuration as $\mathrm{C}$, leading to similar band structures in bulk crystals, while the SOC increases strongly with atomic number $Z$, roughly as $Z^{4}$. This has led to enormous interest in graphene-like, 2D materials of group IV elements. Stanene, along with graphene, silicene and germanene belongs to this group IV 2D materials family. It is a 2D monolayer of $\mathrm{Sn}$ in a honeycomb, graphene-like, lattice. Stanene took its name from the latin name for tin - stannum [5].The interest in stanene can be regarded as a followup of the development of the elemental 2D materials field, in particular the research on the feasibility of the lighter group IV elements - Si and Ge - to form their own 2D structures - silicene and germanene. The investigation of stanene has started in 2013 
with a seminal paper by $\mathrm{Xu}$ et al. [5] Another early report of the properties of free standing stanene was by Broek et al. in 2014 [6].

\subsection{Structural stability}

Like the other novel 2D materials from group IV, stanene has a buckled honeycomb lattice structure with 2 atoms in the primitive cell. The lattice constant has been initially reported to be $4.62-4.68 \AA$ [5] [6]. Sn atoms are relatively large in comparison with the lighter group IV elements. This gives rise to two extraordinary effects, which make stanene a very interesting material. One is buckling of the lattice, and the other is strong spin-orbit coupling. The repulsion forces between atoms are relatively strong which results in larger bond lengths that prevent the atoms from forming strong $\pi$ bonds. $\mathrm{Sn}$ atoms are pushed in the out-of-plane direction, effectively buckling the lattice and deviating the structure away from pure $s p^{2}$ hybridization. The buckling corresponds to a mixed $s p^{2}-s p^{3}$ hybridization. This effect has far reaching consequences and is the main characteristic that distinguishes stanene from graphene. Similar effects are found in silicene and germanene [7]. However, in case of Sn atoms, as the largest among the three, the effects and resulting phenomena are the strongest.

The other distinguishing effect present in stanene is non-negligible spin-orbit coupling (SOC) which makes it a topological insulator [5] [8], analogous to the initial prediction of a QSHI state by Kane and Mele [3]. The SOC is much stronger than in C, Si or Ge, due to the larger nuclear charge of Sn atoms. Therefore, the SOC-induced gap at the K and K' points, which lies at the heart of the formation of the QSHI state, is much larger and has been predicted to surpass the thermal energy even at room temperature.

In a free-standing stanene, similarly to silicene and germanene, there are 2 energy minima classified according to buckling height $\Delta$ as high (HB) and low (LB) buckling of the lattice. Calculations of stability suggest that, in contradiction to silicene and germanene, the HB phase is the global minimum with lattice constant of $3.4 \AA$ [9]. However, that phase is metallic. Hence the interest is mainly in the LB phase, which is predicted to have non-trivial topological properties [5]. In the LB phase, stanene has the lattice constant $\lambda=4.68 \AA$ and buckling height $\Delta=0.85 \AA$ [5]. Fig. 2.1 a and 2.1c show the atomic structure of free-standing LB stanene as well as its predicted structural parameters. Another form of a free-standing monolayer of $\mathrm{Sn}$ is dumbbell stanene, which is also calculated to be more stable than the LB phase [10]. This structure is 
however far from the graphene-like configuration hence it is not going to be discussed further in this review.
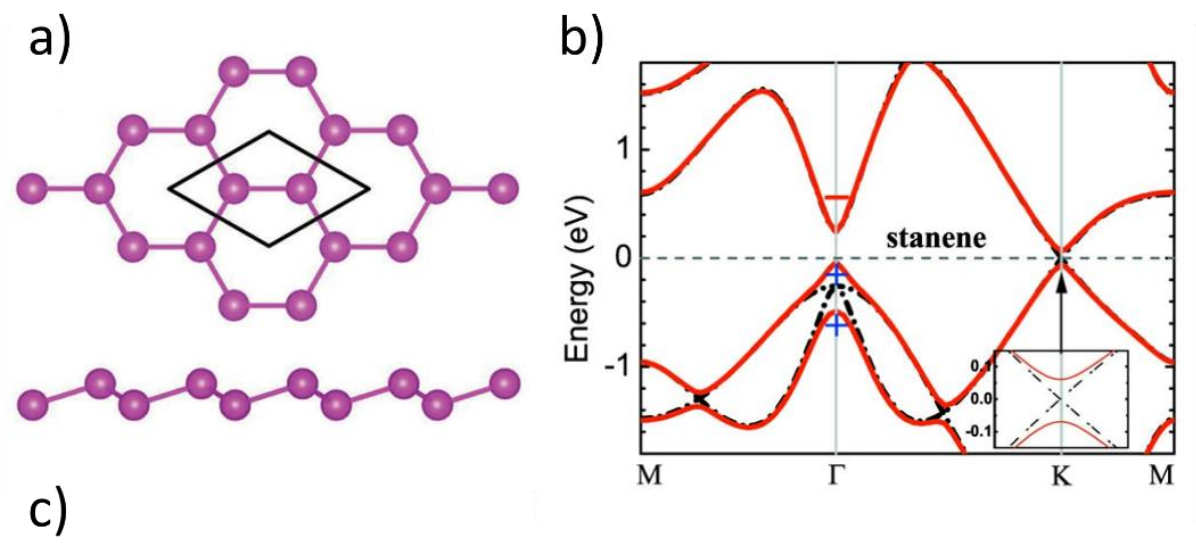

\begin{tabular}{lllll}
\hline & $\mathrm{C}$ & $\mathrm{Si}$ & $\mathrm{Ge}$ & $\mathrm{Sn}$ \\
\hline$a(\AA)$ & 2.468 & 3.868 & 4.060 & 4.673 \\
$d(\AA)$ & 1.425 & 2.233 & 2.344 & 2.698 \\
$\Delta(\AA)$ & 0.00 & 0.45 & 0.69 & 0.85 \\
$v_{\mathrm{F}}\left(10^{6} \mathrm{~m} \mathrm{~s}^{-1}\right)$ & $1.01(0.83)$ & $0.65(0.53)$ & $0.62(0.52)$ & $0.55(0.48)$ \\
$m^{*}\left(m_{0}\right)$ & $0.000(0.000)$ & $0.001(0.001)$ & $0.007(0.008)$ & $0.029(0.028)$ \\
$E_{\mathrm{g}}(\mathrm{meV})$ & $0.0(0.0)$ & $1.9(1.5)$ & $33(24)$ & $101(73)$ \\
\hline
\end{tabular}

Fig. 2.1. a) Top and side view of the stanene lattice with primitive cell indicated by black rhombus. b) Electronic band structure of stanene without (black dash-dotted lines) and with (red solid lines) spin-orbit coupling. The inset shows a zoomed-in energy dispersion near the $K$ point. The Fermi level is indicated by the dashed line. b) Comparison of the structural and electronic parameters of group IV elemental 2D materials. From the top: lattice constant $a$, bond length $d$, buckling parameter $\Delta$, Fermi velocity $v_{F}$, effective masses of Dirac particles $m *$, Electronic band gap $E_{g}$. Electronic quantities are derived from hybrid HSE06 calculations. The GGA results are given in parentheses. $E_{g}$ and $m^{*}$ are calculated with the inclusion of SOC (without SOC, $E_{g}=$ $\left.m^{*}=0\right)$. Reproduced from a) [13], b) [5] c) [17]. 


\subsection{Properties}

Many theoretical studies have been done on a variety of stanene properties. Here we shortly summarize its most important electronic characteristics, as these draw most attention to the material. The two most important features recognized in its band structure are: (i)preservation of the linear energy dispersion (Dirac cones) at the $K$ and $K$ ' points, which signalizes high carrier mobility, as well as (ii) a significant SOC induced band gap of $100 \mathrm{meV}$ [5] which predicts stanene to be a topological insulator and allows for a quantum spin Hall effect (QSHE) to occur at room temperature [16]. This seems to be the largest non-trivial band gap for a free-standing 2D material of group IV, as $\mathrm{Pb}$ - the heaviest group IV element - in its 2D form is a metal [5]. The bandgap can be further tuned and enlarged by various techniques, such as chemical functionalization [11], strain [12] [13] or electrical field [14] [15] up to $0.3 \mathrm{eV}$ [5]. Fig. $2.1 \mathrm{~b}-\mathrm{c}$ give insight in stanene's electronic properties.

\subsection{Substrates}

Experimental realization of stanene (as well as silicene and germanene) is problematic, due to $\mathrm{sp}^{3}$ hybridization being energetically more favorable than $\mathrm{sp}^{2}$. As a result, there is no layered form of $\mathrm{Sn}$ analogous to graphite in nature. The solution to this obstacle is synthesis on a substrate that would support formation of a Sn monolayer. The main challenge in this approach is the choice of a suitable substrate. There are a few properties that a substrate should poses to be a promising candidate. First of all, the stanene substrate heterostructure must be stable. The stability is ensured in 2 ways hexagonal/honeycomb symmetry of the outermost layer of the substrate and small lattice mismatch, in order to minimize the strain in the Sn layer. Secondly, the electronic properties of free standing stanene have to be preserved. To achieve this, preferable substrates must have a band gap as well as interact weakly (via Van der Waals forces) with the 2D material. Therefore, layered semiconductors/insulators with matching lattice constants would be perfect candidates a priori.

\subsubsection{Substrates with reported realization of epitaxial of stanene}

Several substrates have been examined theoretically for their feasibility to support stanene and preserve, or possibly even enhance, its properties. We note in passing that Sn growth on various substrates has been studied for a long time. Only recently however, thanks to the rise of graphene and the prediction of the exotic properties in 
analogous 2D materials, the attention was directed to the quantum physical properties of Sn sheets.

The first report calling an epitaxial multilayer of Sn "stanene" has been reported in 2015 by Zhu et al. [25]. The Sn layer was epitaxially grown on Te-terminated bismuth telluride $\left(\mathrm{Te}_{-} \mathrm{Bi}_{2} \mathrm{Te}_{3}\right)$ (see Fig. 2.1d). The growth was characterized by the VollmerWeber mode (island growth). While the structural properties were in close accordance with the predicted values, the electronic band structure was strongly influenced by the substrate. The report opened the field of epitaxially grown stanene-like structures. Motivated by that successful experimentally realized growth, systematic DFT calculations of the system by Zhang et al. suggested that growth on the Te-terminated $\mathrm{Bi}_{2} \mathrm{Te}_{3}$ surface follows a partial-layer-by-partial-layer (PLBPL) growth mode, characterized by short-range repulsive pairwise interactions of the Sn adatoms. They proposed that $\mathrm{Bi}_{2} \mathrm{Te}_{3}$ pre-covered with a Bi bilayer would support the nucleation-andgrowth mechanism, strongly favoring single crystalline stanene [26]. Finally, a critical view on the possible growth of stanene with its expected properties on this substrate was signaled recently by Li et al. [27]. In their investigation of the electronic band structure of that system by XPS and ARPES, they showed a significant interfacial chemical interaction between $\mathrm{Sn}$ atoms and $\mathrm{Bi}_{2} \mathrm{Te}_{3}$. The presence of this interaction, leading to $\mathrm{Sn}-\mathrm{Te}$ chemical bonds in the stanene $/ \mathrm{Bi}_{2} \mathrm{Te}_{3}$ system, casts doubts on the feasibility of this substrate for stanene growth.

Indium antimonide( $\mathrm{InSb})$ has been regarded as a promising candidate for the growth of Sn sheets [18] [19] [20]. Barfuss et al. [21] reported the possibility to obtain a Quantum 3D topological phase in strained $\alpha$-Sn on this substrate. They also predicted the QSHE in a Sn monolayer on this substrate. Another argument for the feasibility of this material to support a stanene layer was provided by Tang et al. [22]. In their paper they investigated dumbbell stanene on the $\operatorname{InSb}(111)-(2 \times 2)$ thin film and concluded that, despite strong coupling between the substrate and the Sn layer, the topologically nontrivial properties persist in the hybridized structure, such that DB stanene is a $2 \mathrm{D}$ TI without inversion symmetry. Finally $\mathrm{Xu}$ et al. [23] reported on stanene epitaxially grown on $\mathrm{InSb}(111) \mathrm{B}$ ( $\mathrm{Sb}$ terminated) (see Fig. 1g ). ARPES measurements of the pristine films and K-doped films demonstrate a large gap of $0.44 \mathrm{eV}$ at the Brillouin zone center. The size of that band gap is larger than that expected for free-standing stanene, because of the electronic coupling between the Sn and InSb conduction band states [23]. Such a large band gap suggests the exotic properties in this structure to be accessible even above room temperature (For $\mathrm{T}=300 \mathrm{~K}$ the thermal energy $\mathrm{k}_{\mathrm{B}} \mathrm{T}$ is 0.25 $\mathrm{eV}$ ). 
An interesting experiment with a similar substrate was undertaken by Gou et al. [24], who report on an epitaxially grown stanene film on an antimony $\mathrm{Sb}(111)$ substrate (see Fig. 2.1f ). The significant stress, that the stanene layer undergoes, widens the bandgap at the $K$ points to $0.2 \mathrm{eV}$, which is twice as big as the predictions for the free-standing material. However, multiple bands cross the Fermi level at the $\Gamma$ point making stanene metallic. A systematic investigation with STS proved that the stanene band structure can be tuned with strain.

In their study from 2018, Zang et al. [28] report on stanene grown on Sr-doped lead telluride ( $\mathrm{Sr}-\mathrm{PbTe}(111))$. The $\mathrm{Sn}$ layer was unintentionally passivated by $\mathrm{H}$, despite being kept in an ultra-high vacuum (UHV) environment. While the obtained stanene seemed to be a trivial insulator, the authors suggest activating the topological properties by streching its lattice constant, which, as they further imply, can be done using similar substrates, like EuTe, SrTe, BaTe with larger lattice constants. At about the same time, $\mathrm{PbTe}$-covered $\mathrm{Bi}_{2} \mathrm{Te}_{3}$ was used by Liao et al. to grow few-layer stanene films, which revealed novel superconducting behaviors controlled by the number of Sn layers [29].

After the successful growth of silicene on $\operatorname{Ag}(111)$, this became another candidate substrate for the possible growth of monolayer $\mathrm{Sn}$. Ag was one of the metals investigated in theoretical work of Guo et al. [35] about the interfacial properties of stanene on various metals. They concluded that, while stanene is structurally stable on $\mathrm{Ag}$ and some of the other metals examined, it undergoes metallization on all of them. Further theoretical insight into the structural stability of the stanene/ $\operatorname{Ag}(111)$ system was given by Gao et al. [36]. They showed that there are four stable reconstructions which could revert to the free-standing structure upon removal of the Ag substrate. They suggested that it is a good candidate for a proof-of-principle transistor, similarly to the silicene-made device, which was made by etching away Ag without destruction of the overlayer [37]. Inspired by theory, Yuhara et al. [30] reported on the growth of large area planar Sn-sheets on $\mathrm{Ag}$ (111) (see Fig. 2.1a ). Upon deposition of 1/3 ML, an $\mathrm{Ag}_{2} \mathrm{Sn}$ alloy is formed. Further deposition gave rise to an additional planar layer of Sn. ARPES measurements identified the heterostructure as metallic. The formation of an alloy layer poses a question about the possibility to remove the substrate in order to obtain a freestanding material, like in the case of silicene. An attempt to solve this problem was undertaken by Luh et al. [38] who performed a temperature dependent study on Sn growth on $\mathrm{Au}(111)$. Inspired by the previously calculated large diffusion coefficient for $\mathrm{Sn}$ on $\mathrm{Ag}(111)$ at $300 \mathrm{~K}$ they conducted $\mathrm{Sn}$ deposition at lowered temperatures in order to prevent forming an alloy. However, they failed to achieve this even at temperatures as low as $96 \mathrm{~K}$. The question if there is an alloy-free regime below that temperature in the $\mathrm{Sn} / \mathrm{Ag}$ systems remains unanswered for now. 
A similar example of a planar $\mathrm{Sn}$ layer was reported on $\mathrm{Cu}(111)$ by Deng et al. [33] (see Fig. 2.1e ). The method used to prevent forming an alloy was deposition at low temperature, in this case $200 \mathrm{~K}$. The obtained structure was an atomic layer of $\mathrm{Sn}$ in a planar honeycomb lattice with no buckling, which was named "ultraflat stanene". The strong interactions with the substrate and the lattice stretching stabilized the zero buckling geometry, creating a honeycomb lattice. This lattice was characterized by an $s$ $-p$ band inversion and a bandgap at the $\Gamma$ point induced by SOC. Green's function calculations confirmed the topologically derived boundary states suggested by scanning tunneling spectroscopy ( STS ) measurements. The structure is stable up to $240 \mathrm{~K}$. It is also important to note that even though there was a finite bandgap in the stanene layer, ARPES measurements showed that the system was overall metallic, due to metallic bands from the substrate.

Nigam et al. [39] investigated the $\mathrm{Au}(111)$ surface as a potential candidate for a substrate supporting stanene via density functional theory (DFT) calculations. The $\mathrm{Sn}$ layer prefers a planar over a buckled structure, which was explained by the participation of $\mathrm{Sn}-\mathrm{p}_{\mathrm{z}}$ orbitals in bonding with $\mathrm{Au}$. This is contradictory to the work by Guo et al. [35], who in their calculations obtained buckled stanene on $\mathrm{Au}(111)$ with a buckling parameter almost twice bigger than in the case of free-standing stanene. The authors realized this discrepancy and suggested different Van der Waals corrections considered in the two reports [35] as the possible reason. Experimental realization of stanene on $\mathrm{Au}(111)$ was achieved by Liu et al. [40] The deposited $\mathrm{Sn}$ atoms first formed a surface $\mathrm{Sn}-\mathrm{Au}$ alloy, with a coverage-dependent structure. Upon further deposition, above a critical coverage, the Au-Sn alloy was converted into a strongly buckled, disordered honeycomb lattice of epitaxial stanene. DFT calculations and STM images suggest strong tensile strain in the layer. Maniraj et al. [31], after their initial report on the same alloy [41], also reported on stanene formation on $\mathrm{Au}(111)$ (see Fig. 2.1b). Their ARPES investigation showed that the band structure of the $\mathrm{Sn} / \mathrm{Au}$ superstructure is dominated by a linearly dispersing band centered at the $\Gamma$ point, corresponding to a high Fermi velocity and a spin texture of a three-dimensional topological insulator.

Glass et al. investigated silicon carbide ( $\mathrm{SiC}$ ) substrates and reported the first realization of a triangular $\mathrm{Sn}$ lattice on $\mathrm{SiC}(0001)$ [45]. STM revealed the structure of the Sn lattice, which was reproduced in DFT calculations. DFT predicted a Sn-related band to cross the Fermi level, in the middle of a large band gap. Additional UPS measurements suggest a Mott-Insulating state. Encouraged by this promising result, Matusalem et al. [46] showed in their calculations that the passivation of the substrate and Sn layer in the stanene/SiC system results in quasi freestanding overlayers, with the presence of linearly dispersing Dirac-like bands in the band structures. They also identified the 
configuration in which stanene conserves its topological character. Another variation, proposed by the same group was a graphene-covered $\mathrm{SiC}$ substrate [47]. In this case, while the Dirac cone is conserved, the authors report on strong charge transfer from stanene to graphene, which makes the topology of the system unclear. The possibility of substrate engineering was significantly explored by Di Sante et al. [48], who analyzed the influence of an elemental buffer layer on the stanene/SiC system. The buffer layers they examined were elemental Group III and V lattices. The goal was to protect the QSHI state in stanene. They chose atoms which have their bonding and antibonding states energetically far away from the chemical potential in order to minimize the detrimental staggered potential (due to lifting sublattice degeneracy). The analysis made them propose $\mathrm{P}$ and As as the best candidates for buffer layers. While other attempts focused on adding additional layers to tune the substrate, Ferdous et al. [49] investigated the properties of a stanene/SiC heterobilayer (see Fig. 2.3a). That system manifests a wide band gap up to $160 \mathrm{meV}$ at the $K$ point with a well-preserved Dirac cone. A closer look at the topological properties of stanene on $\mathrm{SiC}$ was undertaken by $\mathrm{Li}$ [50]. In this calculations stanene was stabilized by the strong interfacial bonding. This resulted in quadratically dispersing (non-Dirac) topological states at the $\Gamma$ point, constructed of stanene's $p_{x, y}$ instead of the $p_{z}$ orbitals. 
a)

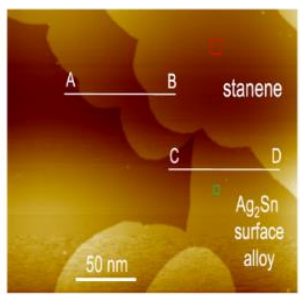

c)

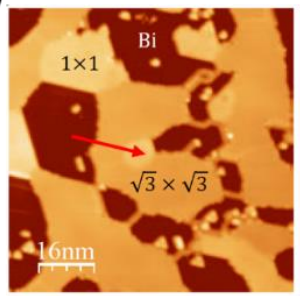

e)

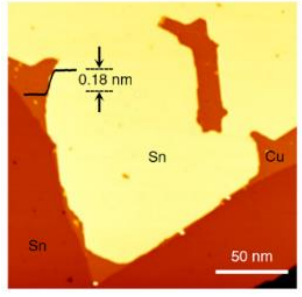

g) b)
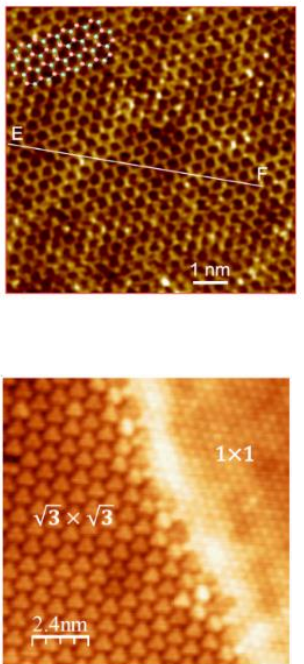

d)
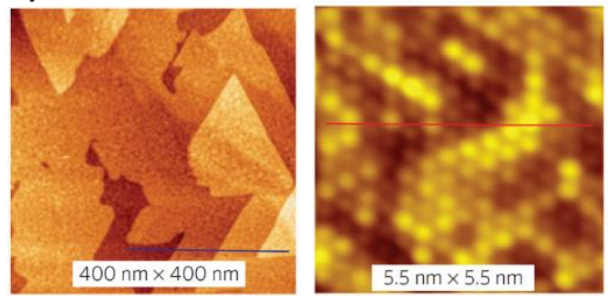

f)
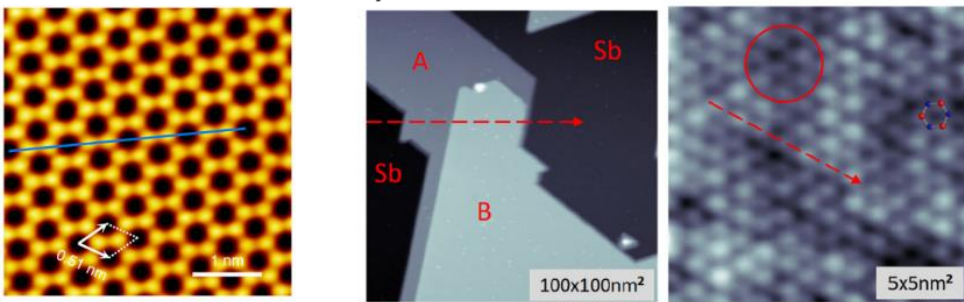

h)
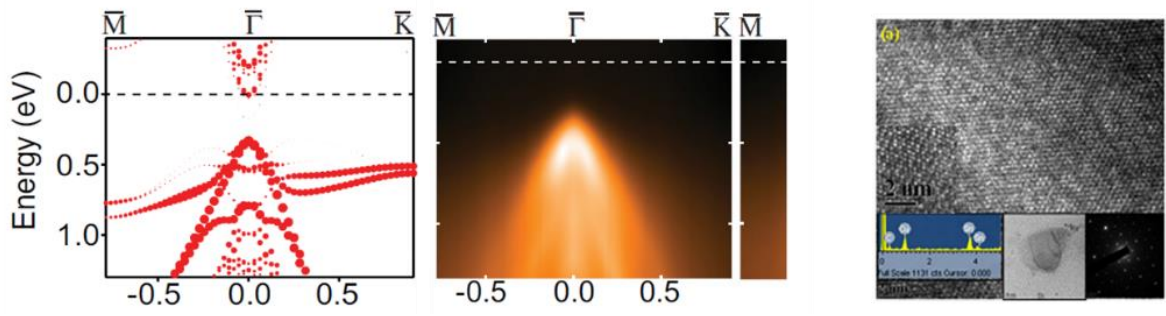

Fig. 2.2: (a-f)Selected large area and high resolution STM images of epitaxial stanene grown on: a) $\mathrm{Ag}_{2} \mathrm{Sn}$ surface alloy b) $\mathrm{Au}(111)$ c) $\mathrm{Bi}(111)$ d) $\mathrm{Bi}_{2} \mathrm{Te}_{3}$ e) $\left.\mathrm{Cu}(111) \mathrm{f}\right) \mathrm{Sb}$ (111) g) Calculated (left) and measured with ARPES(right) band structure of stanene on InSb. h) HRTEM image of a free-standing Sn layer showing a hexagonal lattice. Reproduced from a) [30] b) [31] c) [32] d) [25] e) [33] f [24] g) [23] h) [34]. 
While epitaxial growth is the common way to approach synthesis of stanene, Saxena et al. [34] took a different path and reported growing a freestanding few-layer stanene film, obtained by impinging pulses from a tunable Ti:Saphire ultra-fast femto-second laser onto a target in a liquid medium (see Fig. 2.1h). They performed a series of structural studies which seem to confirm the claim, however no electronic properties measurements have been reported so far. Their further report confirms stanene's structural signature in optical measurements [51].

Reports on experimentally realized growth of silicene [52] [53] and germanene [54] on molybdenum disulfide $\left(\mathrm{MoS}_{2}\right)$ gave hopes for utilizing the material as a substrate also for epitaxial synthesis of stanene. Liang et al. [55], Xiong et al. [56] and Ren et al. [57] (see Fig. 2.3b ) reported, at approximately the same time, very similar studies on the stability and electronic properties of a stanene/ $\mathrm{MoS}_{2}$ heterobilayer using DFT. A band gap was opened at the $K$ point (slightly shifted in the case of Ren et al.) due to $\mathrm{MoS}_{2}$ breaking the symmetry of the sublattices of stanene (slightly bigger in the case of Xiong et al. than in the other two reports). In the case of Liang et al. and Ren et al., the Dirac cone was preserved, while Xiong et al. report on electron transfer from stanene to the substrate forming an internal electric field. The Dirac cone recovers upon applying an external field, equal to the internal one. The reason for the discrepancies may come from a different optimal interlayer spacing calculated, different stacking patterns as well as different functionals used in the calculations. All three reports conclude that the band gap can be effectively tuned with external strain and electric field. In the experimental field, although Chen et al. [58] report on forming multilayer stanene on $\mathrm{MoS}_{2}$. The thickness of the deposited layer being $100 \mathrm{~nm}$, poses a question if the name "stanene", which refers to an atomic monolayer of the material, is used appropriately, as the experimental measurements in the paper are limited to TEM and XRD, while AFM images are not atomically resolved.

There are several more theoretical papers on different substrates, that are predicted to support stanene. Up to now however, there has been no experimental realization of stanene on any of them reported in the literature. 
Table 2.I: Substrates on which stanene growth has been reported. In the columns respectively: a substrate, substrate general electronic properties, reconstruction, stanene lattice constant $\lambda$, buckling height $\Delta$

\begin{tabular}{|c|c|c|c|c|c|}
\hline Substrate & $\begin{array}{c}\text { Substrate Electr. } \\
\text { Prop. }\end{array}$ & Reconstructions & $\lambda[\AA]$ & $\Delta[\AA]$ & Source \\
\hline $\mathrm{Bi}_{2} \mathrm{Te}_{3}$ & $\begin{array}{l}\text { Topological } \\
\text { insulator }\end{array}$ & $1 \times 1$ & 4.4 & 3.5 & [25] \\
\hline $\begin{array}{c}\text { Free- } \\
\text { standing }\end{array}$ & No substrate & $1 \times 1$ & 4.7 & 3.3 & [34] \\
\hline $\mathrm{Sb}(111)$ & semimetal & $1 \times 1$ & 4.3 & & [24] \\
\hline $\mathrm{Cu}(111)$ & metal & $2 \times 2$ & 5.1 & 1.8 & [33] \\
\hline $\begin{array}{l}\mathrm{InSb} \\
(111)\end{array}$ & semiconductor & $1 \times 1$ & 4.58 & 2.85 & [23] \\
\hline $\mathrm{Bi}(111)$ & metal & $\begin{array}{c}1 \times 1 \\
\sqrt{3} \times \sqrt{3} \mathrm{R} 30\end{array}$ & 4.54 & 4 & [32] \\
\hline $\mathrm{MoS}_{2}$ & semiconductor & & & 2.9 & [58] \\
\hline $\mathrm{Au}(111)$ & metal & $\begin{array}{c}2 \times 2, \sqrt{3} \times \sqrt{3}, \\
\sqrt{3} \times \sqrt{7}->1 \times 2 \text { of } \\
\text { stanene } \\
\sqrt{7}\end{array}$ & $\begin{array}{l}5.1- \\
5.7\end{array}$ & 2.4 & [31] \\
\hline $\mathrm{Ag}(111)$ & metal & $\sqrt{3} \times \sqrt{3}$ & 4.98 & 2.6 & [30] \\
\hline
\end{tabular}




\subsubsection{Substrates theoretically predicted to support epitaxial stanene}

Hexagonal boron nitride (hBN), as an insulator with Van der Waals structure, was an obvious candidate for a stanene substrate. A theoretical study on the stability and the effect of strain was undertaken by Wang et al. [13] (see Fig. 2.4c ). They showed that stanene is stable on hBN and slightly compressed with respect to its freestanding form, which makes it semi-metallic. However, by application of an external strain, a QSHI phase can be induced. Additionally, the authors found another configuration with smaller lattice mismatch, which exhibits a QSHI phase without any strain. Another theoretical configuration was proposed in the paper of Wang et al. [59]. The result suggests that hBN can support stanene and preserve a topological gap. Khan et al. [60] also reported on a stanene/hBN heterobilayer in their theoretical work. The authors propose yet another stable configuration, in which the stanene layer is slightly stretched. The band structure of the $\mathrm{Sn} / \mathrm{hBN}$ heterobilayer shows a direct band gap of about 30 $\mathrm{meV}$ at the Fermi energy, while the linear Dirac dispersion relation is maintained. Tensile strain and interlayer distance also have an impact on the band structure, showing that it is very prone to tuning. DFT studies on hexagonal nitrides (AlN, GaN, BN) as stanene substrates were also performed by Yelgel [61]. In the case of $h B N$, the author reported that the Dirac cone in monolayer stanene adsorbed on BN was preserved. The PDOS confirms previous claims that the carrier transport occurs only through the stanene layer. The biggest band gap was calculated in the case of stanene/GaN heterostructure. AlN shows strong interaction with the stanene layer, confirmed by previous reports.

The germanium ( $\mathrm{Ge}(111)$ ) surface was examined by Fang et al.[62] by means of DFT calculations (see Fig. 2.4b). They showed that stanene on $\mathrm{Ge}(111)$ has nontrivial topological phases. The Dirac cone exists around the $\Gamma$ point, and the SOC open a band gap. In their report, El Bachra et al. [64] presented detailed calculations on stability, proving that buckled stanene is the preferable phase. They also suggested that there is a strong interaction between the Sn layer and the substrate. They confirmed that stanene preserves non-trivial topological features. While the theoretical field seem to paint a tempting landscape for further exploration, the experimental reports on $\mathrm{Sn} / \mathrm{Ge}(111)$ heterostructures suggest that a $\mathrm{Sn}$ adlayer rather different from stanene is formed [65] [66]. 
a)

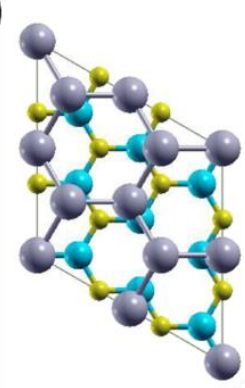

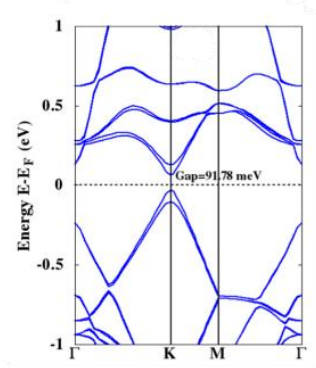

b)
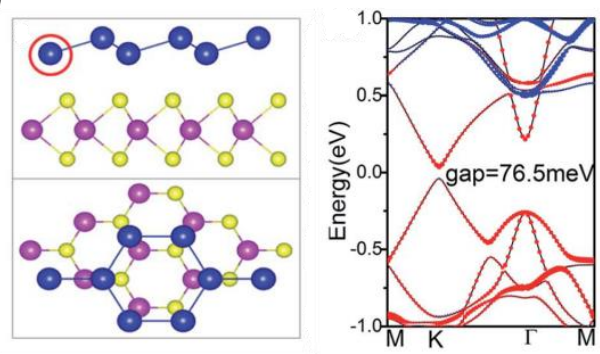

c)
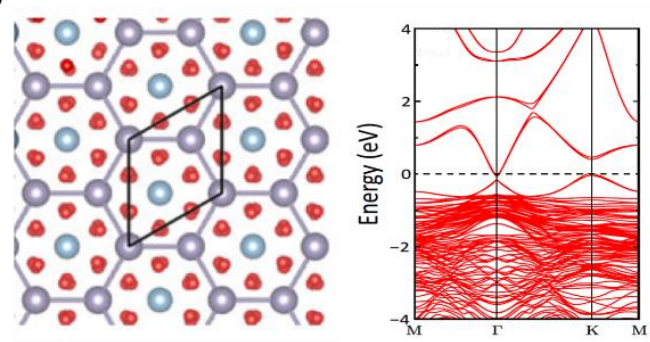

Fig. 2.3: a) Stanene on SiC stack with corresponding calculated band structures with SOC. b) Stanene/MoS $\mathrm{M}_{2}$ stack with the band structure with SOC. c) proposed atomic configuration of stanene on $\alpha-\mathrm{Al}_{2} \mathrm{O}_{3}(0001)$ and their calculated band structures, including SOC. Figures adapted from a) [49] b) [57] c) [43].

An interesting idea is to combine two elemental 2D materials together. Chen et al. [67] explored that idea by investigating a stanene/graphene heterostructure. They studied structural, electronic and optical properties of several stacking configurations by means of DFT. They found interactions between the layers being stronger than typical Van der Waals bonds, which improves the stability of the system. In the case of some stacking configurations the system exhibits a Dirac feature in the vicinity of the $K$ point. The authors suggest the tunability of the gap by means of an electric field. Finally, the system showed enhanced visible light absorption in all configurations. Wu et al. [63] performed similar calculations, additionally showing that multiple crystalline phases can coexist at room temperature (see Fig. 2.4a). They also suggest the possibility of tuning the electronic properties. Yun et al. [68] took a closer look on that aspect and calculated the behavior of the stanene/graphene system under strain and the influence of water vapor 
adsorption. In their stability studies, they report an additional stacking configuration, not mentioned in previous studies. They concluded that the Fermi level can host Dirac or parabolic bands (or a mixture of both) depending on the strain and rotation. They also selected configurations in which a gap can be opened by application of external stress. The calculation on water vapor adsorption showed that it will modulate conductivity but not distort the band structure significantly. Another combination of stanene and graphene together was proposed by Mondal et al. [69] who proposed stanene sandwiched between two graphene layers. The structure exhibits a topologically protected hybrid state at the stanene-graphene interface, which is robust against severe strain.

The interests of Noshin et al. [70] were directed to another elemental 2D material silicene. The authors examine a stanene/silicene bilayer by DFT. Their focus was on investigating thermal conductivity of the bilayer, and they showed that it has a thermal conductivity smaller than that of any other group IV elemental 2D material. Barhoumi et al. [71] showed in their studies that a stanene/silicene bilayer is stable and the relaxed structure is a direct band gap semiconductor.

Guo et al. [35] surveyed several metallic substrates using DFT, including aforementioned $\mathrm{Ag}, \mathrm{Au}, \mathrm{Cu}, \mathrm{Al}$, as well as $\mathrm{Pd}, \mathrm{Pt}$, Ir, and Ni. The stability of the honeycomb lattice was preserved, however buckling parameters changed, depending on the substrate. They also reported on the destruction of the stanene band structure and its metallization for all the substrates examined. Ding et al. [72] proposed InSe and GaTe as other candidates for stanene substrates. In their paper they calculated that stanene is stable on both of the substrates and preserves its Dirac cone as well as the SOC -induced band gap.

Zhang el al.[73] reported calculations on a functionalized stanene/ $\mathrm{PbI}_{2}$ heterostructure as a suitable system for observing the quantum anomalous Hall effect (QAHE). The substrate is non-magnetic, therefore they induced ferromagnetism in the structure via functionalization of stanene. They also proposed a device comprising stanene sandwiched between $\mathrm{PbI}_{2}$ layers. In a follow - up paper [74] the group also showed that the QSHE is present in the same structure. Ni et al. [75] investigated $\mathrm{PbI}_{2}$ and $\mathrm{CaI}_{2}$ by means of DFT. They found both substrates to support stanene. While on $\mathrm{PbI}_{2}$ it exhibits a trivial band gap, on $\mathrm{CaI}_{2}$ it is metallic, but external strain can open a non-trivial band gap. 
Chen et al. [76] investigated theoretically stanene on $\mathrm{WS}_{2}$. They examined the interlayer distance, strain and the influence of an external electric field on the system. The system is stable, above a certain interlayer distance, and the electronic structure has a band gap with a Dirac cone at the $K$ point. The authors further show that the size of the bandgap could be tuned with all the aforementioned parameters. Cao et al. [77] reported on their theoretical investigation on structural stability, as well as electronic and optical properties of a stanene/ZnO heterostructure. After finding the optimal interlayer distance, the electronic structure was determined to be metallic. A band gap can be opened however, by application of electric field and strain. They also showed that the system has potentially high UV absorption capability. Chakraborty et al. [78] investigated a stanene/ $\mathrm{BeO}$ heterostructure. Its structural stability was confirmed, and electronic properties calculations showed a large band gap with Dirac cone opened, the size of which was enlarged by the interaction with the substrate. 

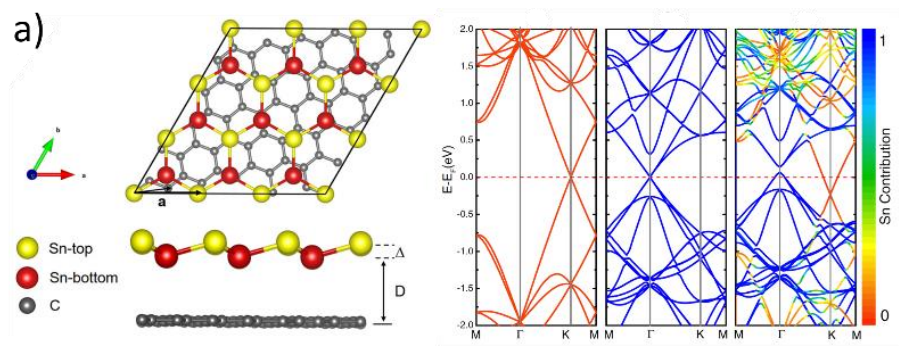

b)

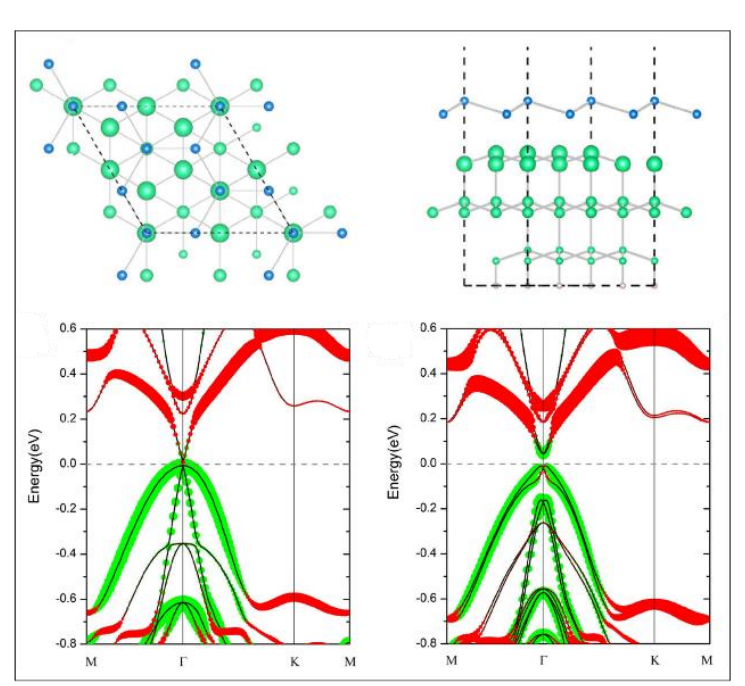

c)

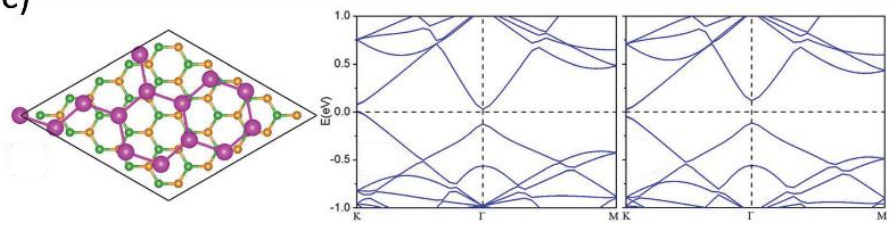

Fig. 2.4: a) Stanene on graphene with band structures of (from the left) graphene, stanene and the whole system. b) Model of the stanene/Ge(111) heterostructure and the band structure (a) without and (b) with SOC. c) Stanene/hBN heterostructure with corresponding band structure without (left) and with (right) SOC. Figures reproduced from a) [63] b) [62] c) [13]. 
Table 2.II: Substrates proposed in DFT calculations. In the columns respectively: a substrate, substrate band gap given in the source article, reconstruction stack (stanene/substrate) used for calculations, an optimized interlayer distance $d$, lattice mismatch $\varepsilon$, buckling height $\Delta$, band gap of the stanene/substrate system, topological $\mathrm{Z}_{2}$ invariant.

\begin{tabular}{|c|c|c|c|c|c|c|c|c|}
\hline Substrate & $\begin{array}{c}E_{\mathrm{g}}^{\text {subs }} \\
(\mathrm{eV})\end{array}$ & reconstruction & $\varepsilon[\%]$ & $d[\AA]$ & $\Delta[\AA \AA]$ & $\mathbf{E}_{\mathrm{g}}[\mathrm{meV}]$ & $\begin{array}{l}Z \\
2 \\
\end{array}$ & source \\
\hline \multirow[t]{4}{*}{$\mathrm{hBN}$} & \multirow[t]{4}{*}{5} & $\begin{array}{l}1 \times 1 / \sqrt{ } 3 \times \sqrt{3} \\
\sqrt{7} \times \sqrt{ } 7 / 5 \times 5\end{array}$ & $\begin{array}{l}6.9 \\
1.5\end{array}$ & 3.30 & & up to 53 & 1 & [13] \\
\hline & & $\begin{array}{c}3 \times 3 \\
/ \sqrt{ } 31 \times \sqrt{ } 31 \mathrm{R} 9\end{array}$ & 0.25 & 3.3 & & 3 & 1 & [59] \\
\hline & & $2 \times 2 / 4 \times 4$ & 7 & $\begin{array}{c}3.7- \\
3.8\end{array}$ & & 30 & & [60] \\
\hline & & $2 \times 2 / 4 \times 4$ & 6 & 3.4 & & 93 & & [61] \\
\hline \multirow[t]{2}{*}{ AlN } & \multirow[t]{2}{*}{2.9} & $\begin{array}{c}1 \times 1 / \sqrt{ } 3 \times \sqrt{ } 3 \\
2 \times 2 / 3 \times 3\end{array}$ & $\begin{array}{l}19.5 \\
3.4\end{array}$ & $\begin{array}{c}2.70 \\
>4.00\end{array}$ & & $\begin{array}{l}\text { semimet. } \\
63\end{array}$ & & [13] \\
\hline & & $2 \times 2 / 3 \times 3$ & 1 & 3 & 0.72 & & & [61] \\
\hline \multirow[t]{2}{*}{ Ge (111) } & \multirow[t]{2}{*}{0.7} & $\sqrt{3} \times \sqrt{3} / 2 \times 2$ & $<1$ & & \multirow[b]{2}{*}{1.4} & \multirow[t]{2}{*}{34} & \multirow[t]{2}{*}{1} & [62] \\
\hline & & $\sqrt{ } 3 \times \sqrt{ } 3 / 2 \times 2$ & 1.5 & 3.2 & & & & [64] \\
\hline InSe & & $\sqrt{3} \times \sqrt{3} / 2 \times 2$ & 0.7 & 3.10 & & 20 & & [72] \\
\hline GaTe & & $\sqrt{3} \times \sqrt{3} / 2 \times 2$ & 2.2 & 3.45 & & 80 & & [72] \\
\hline \multirow[t]{2}{*}{$\begin{array}{c}\alpha-\mathrm{Al}_{2} \mathrm{O}_{3} \\
(0001)\end{array}$} & \multirow[t]{2}{*}{ Ins. } & $1 \times 1 / 1 \times 1$ & & 2.88 & \multirow[t]{2}{*}{$\begin{array}{l}0.7- \\
1.2\end{array}$} & 250 & 1 & [44] \\
\hline & & $1 \times 1 / 1 \times 1$ & 2.7 & $\begin{array}{c}2.7- \\
29\end{array}$ & & 260 & & [43] \\
\hline \multirow[t]{2}{*}{$\operatorname{Ag}(111)$} & \multirow[t]{2}{*}{-} & $\begin{array}{c}7 \times 7 R 19.107 / \\
\sqrt{ } 19 \times \\
\sqrt{ } 19 R 23.42\end{array}$ & 3.3 & $\begin{array}{l}2.41- \\
2.48\end{array}$ & & & & [36] \\
\hline & & $\begin{array}{c}4 \times 4 / \sqrt{ } 43 \times \\
\sqrt{ } 43 R 7.6\end{array}$ & 2.7 & 2.49 & 1.06 & metallic & & [35] \\
\hline
\end{tabular}


Substrates feasible for epitaxial growth of stanene

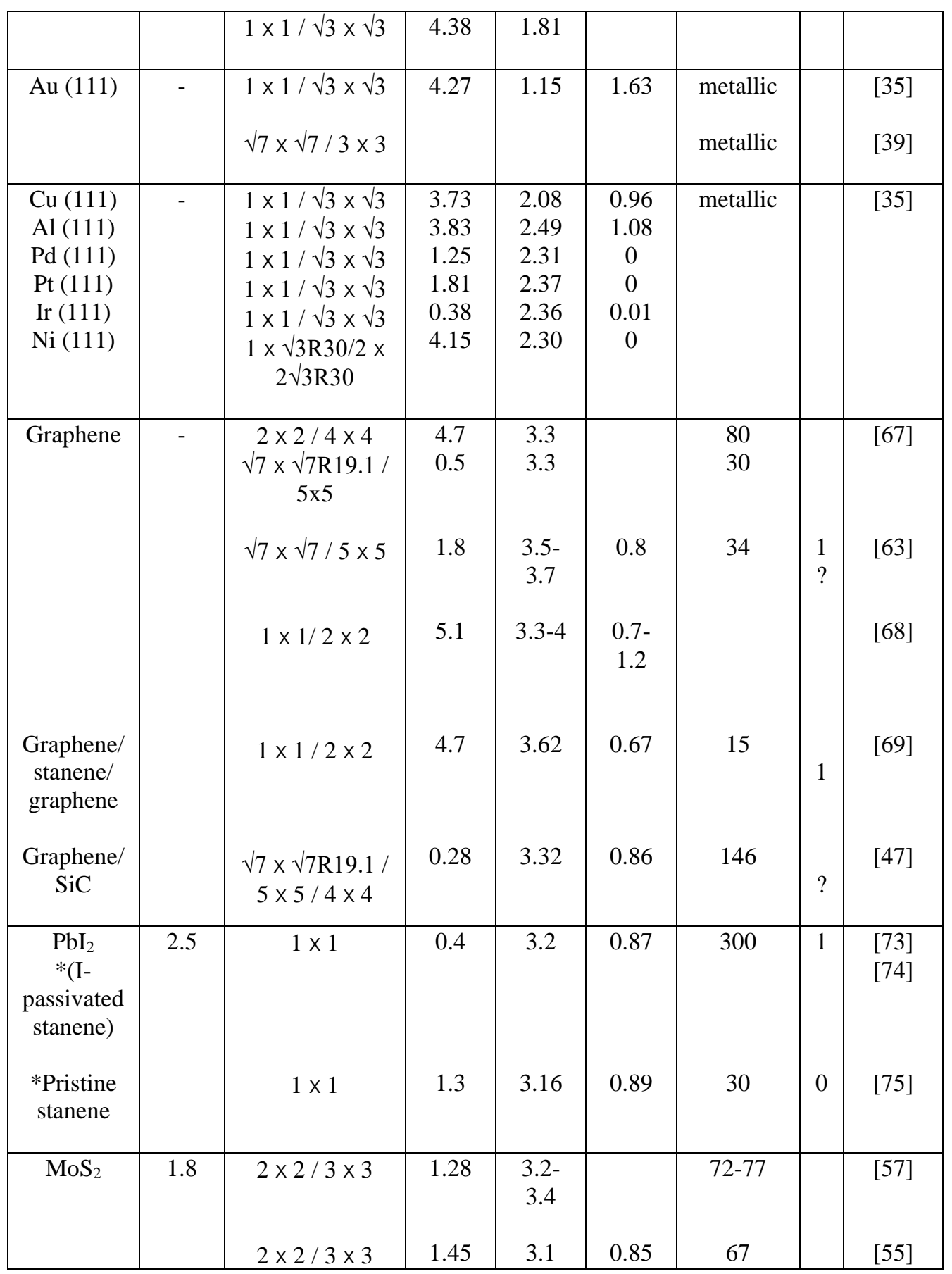




\begin{tabular}{|c|c|c|c|c|c|c|c|c|}
\hline & & $2 \times 2 / 3 \times 3$ & 1.1 & 2.8 & & 64 & & [56] \\
\hline $\mathrm{CaI}_{2}$ & & $1 \times 1$ & 2.1 & 3.35 & 0.9 & metallic & * & [75] \\
\hline $\mathrm{WS}_{2}$ & 1.8 & $2 \times 2 / 3 \times 3$ & 1.9 & 3.00 & 0.85 & 62.9 & & [76] \\
\hline $\mathrm{ZnO}$ & 1.7 & $2 \times 2 / 3 \times 3$ & 3.3 & 3.00 & 0.78 & $\begin{array}{c}\text { metallic } \\
\text { (60 at } 3.2 \\
\text { A) }\end{array}$ & & [77] \\
\hline $\begin{array}{c}\mathrm{SiC} \\
\mathrm{SiC}-\mathrm{H} \\
\mathrm{SiC}-\mathrm{F}\end{array}$ & 2.5 & $\begin{array}{l}2 \times 2 / 3 \times 3 \\
2 \times 2 / 3 \times 3 \\
2 \times 2 / 3 \times 3\end{array}$ & $\begin{array}{l}0.46 \\
7.05\end{array}$ & $\begin{array}{l}2.16 \\
4.33 \\
4.69\end{array}$ & & $\begin{array}{c}\text { metallic } \\
100 \\
160\end{array}$ & $\begin{array}{l}1 \\
1\end{array}$ & [46] \\
\hline $\mathrm{SiC} M L$ & & $2 \times 2 / 3 \times 3$ & 1 & $\begin{array}{l}3.19- \\
3.21\end{array}$ & & 154-162 & & [49] \\
\hline $\begin{array}{c}\mathrm{SiC} \\
(0001)\end{array}$ & & $2 \times 2 / 3 \times 3$ & 1 & $\begin{array}{l}2.0- \\
2.7\end{array}$ & $\begin{array}{c}0.4- \\
1.4\end{array}$ & 23 & & [50] \\
\hline Silicene & & $4 \times 4 / 5 \times 5$ & 0.6 & $\begin{array}{l}3.4 \\
2.65\end{array}$ & $\begin{array}{l}0.83 \\
0.0\end{array}$ & 70-160 & & $\begin{array}{l}\text { [70] } \\
\text { [71] }\end{array}$ \\
\hline $\mathrm{BeO}$ & 5.0 & $2 \times 2 / 3 \times 3$ & 7 & 3.00 & 0.86 & 98 & & [78] \\
\hline
\end{tabular}

\subsection{Conclusions}

In summary, the field of stanene has many promises to realize and obstacles to overcome. The main promise is a QSHE at room temperature, and all the possible utilizations of that effect in various devices. The main obstacle is synthesis. The consensus in the community is that freestanding stanene is not possible to realize, and techniques that would support the growth on some kind of supporting structure are necessary. The main technique utilized so far in order to create stanene is epitaxial growth on a solid crystalline substrate. This review summarized the substrates examined by theoretical calculations for their feasibility of supporting a stanene layer. It also includes many up-to-date reports on experimental realizations of stanene-like single layers of Sn. This report thus serves as a useful "orientation point" for researchers that are new in the field. 


\section{References}

[1] K. S. Novoselov et al., "Two-dimensional gas of massless Dirac fermions in graphene," Nature, vol. 438, no. 7065, pp. 197-200, Nov. 2005, doi: 10.1038/nature04233.

[2] P. J. Zomer, S. P. Dash, N. Tombros, and B. J. van Wees, "A transfer technique for high mobility graphene devices on commercially available hexagonal boron nitride," Appl. Phys. Lett., vol. 99, no. 23, p. 232104, Dec. 2011, doi: 10.1063/1.3665405.

[3] C. L. Kane and E. J. Mele, "Quantum Spin Hall Effect in Graphene," Phys. Rev. Lett., vol. 95, no. 22, p. 226801, Nov. 2005, doi:

10.1103/PhysRevLett.95.226801.

[4] Y. Yao, F. Ye, X.-L. Qi, S.-C. Zhang, and Z. Fang, "Spin-orbit gap of graphene: First-principles calculations," Phys. Rev. B, vol. 75, no. 4, p. 041401, Jan. 2007, doi: 10.1103/PhysRevB.75.041401.

[5] Y. Xu et al., "Large-Gap Quantum Spin Hall Insulators in Tin Films," Phys. Rev. Lett., vol. 111, no. 13, p. 136804, Sep. 2013, doi:

10.1103/PhysRevLett.111.136804.

[6] B. van den Broek, M. Houssa, E. Scalise, G. Pourtois, V. V. Afanas 'ev, and A. Stesmans, "Two-dimensional hexagonal tin: ab initio geometry, stability, electronic structure and functionalization," 2D Mater., vol. 1, no. 2, p. 021004, Aug. 2014, doi: 10.1088/2053-1583/1/2/021004.

[7] S. Cahangirov, M. Topsakal, E. Aktürk, H. Şahin, and S. Ciraci, "Two- and One-Dimensional Honeycomb Structures of Silicon and Germanium," Phys. Rev. Lett., vol. 102, no. 23, p. 236804, Jun. 2009, doi: 10.1103/PhysRevLett.102.236804.

[8] M. Ezawa, "Monolayer Topological Insulators: Silicene, Germanene, and Stanene," J. Phys. Soc. Jpn., vol. 84, no. 12, p. 121003, Dec. 2015, doi: 10.7566/JPSJ.84.121003.

[9] P. Rivero, J.-A. Yan, V. M. García-Suárez, J. Ferrer, and S. Barraza-Lopez, "Stability and properties of high-buckled two-dimensional tin and lead," Phys. Rev. B, vol. 90, no. 24, p. 241408, Dec. 2014, doi: 10.1103/PhysRevB.90.241408. 
[10] P. Tang et al., "Stable two-dimensional dumbbell stanene: A quantum spin Hall insulator," Phys. Rev. B, vol. 90, no. 12, p. 121408, Sep. 2014, doi:

10.1103/PhysRevB.90.121408.

[11] Y. Mao, L. Long, C. Xu, and J. Yuan, "First-principles study on the structure and electronic properties of stanane under electric fields," Mater. Res. Express, vol. 5, no. 6, p. 065023, Jun. 2018, doi: 10.1088/2053-1591/aac684.

[12] M. Modarresi, A. Kakoee, Y. Mogulkoc, and M. R. Roknabadi, "Effect of external strain on electronic structure of stanene," Comput. Mater. Sci., vol. 101, pp. 164-167, Apr. 2015, doi: 10.1016/j.commatsci.2015.01.039.

[13] D. Wang, L. Chen, X. Wang, G. Cui, and P. Zhang, "The effect of substrate and external strain on electronic structures of stanene film," Phys. Chem. Chem. Phys., vol. 17, no. 40, pp. 26979-26987, 2015, doi: 10.1039/C5CP04322K.

[14] M. Fadaie, N. Shahtahmassebi, and M. R. Roknabad, "Effect of external electric field on the electronic structure and optical properties of stanene," Opt. Quantum Electron., vol. 48, no. 9, p. 440, Sep. 2016, doi: 10.1007/s11082-016-07095 .

[15] C.-C. Ren, W.-X. Ji, C.-W. Zhang, P. Li, and P.-J. Wang, "The effects of biaxial strain and electric field on the electronic properties in stanene," Mater. Res. Express, vol. 3, no. 10, p. UNSP 105008, Oct. 2016, doi: 10.1088/2053$1591 / 3 / 10 / 105008$.

[16] C.-C. Liu, H. Jiang, and Y. Yao, "Low-energy effective Hamiltonian involving spin-orbit coupling in silicene and two-dimensional germanium and tin," Phys. Rev. B, vol. 84, no. 19, p. 195430, Nov. 2011, doi:

10.1103/PhysRevB.84.195430.

[17] L. Matthes, O. Pulci, and F. Bechstedt, "Massive Dirac quasiparticles in the optical absorbance of graphene, silicene, germanene, and tinene," J. Phys. Condens. Matter, vol. 25, no. 39, p. 395305, Oct. 2013, doi: 10.1088/0953-8984/25/39/395305.

[18] T. Eguchi, J. Nakamura, and T. Osaka, "Structure and Electronic States of the $\alpha$-Sn(111)-( 2×2 ) Surface," J. Phys. Soc. Jpn., vol. 67, no. 2, pp. 381-384, Feb. 1998, doi: 10.1143/JPSJ.67.381. 
[19] B. F. Mason and B. R. Williams, "Growth and recovery of a-Sn on $\mathrm{InSb}(\mathrm{OO} 1)$ using He scattering and LEED," p. 11.

[20] P. Fantini, C. Mariani, and M. Sancrotti, "a-Sn pseudomorphic growth on InSb (111) and (111) surfaces: a high-resolution photoemission study," Surf. Sci., p. 9, 2000 .

[21] A. Barfuss et al., "Elemental Topological Insulator with Tunable Fermi Level: Strained $\alpha$-Sn on InSb(001)," Phys. Rev. Lett., vol. 111, no. 15, p. 157205, Oct. 2013, doi: 10.1103/PhysRevLett.111.157205.

[22] P. Tang et al., "Stable two-dimensional dumbbell stanene: A quantum spin Hall insulator," Phys. Rev. B, vol. 90, no. 12, p. 121408, Sep. 2014, doi: 10.1103/PhysRevB.90.121408.

[23] C.-Z. Xu et al., "Gapped electronic structure of epitaxial stanene on InSb(111)," Phys. Rev. B, vol. 97, no. 3, p. 035122, Jan. 2018, doi: 10.1103/PhysRevB.97.035122.

[24] J. Gou et al., "Strain-induced band engineering in monolayer stanene on Sb(111)," Phys. Rev. Mater., vol. 1, no. 5, p. 054004, Oct. 2017, doi: 10.1103/PhysRevMaterials.1.054004.

[25] F. Zhu et al., "Epitaxial growth of two-dimensional stanene," Nat. Mater., vol. 14 , no. 10 , pp. $1020-1025$, Oct. 2015 , doi: $10.1038 /$ nmat 4384 .

[26] L. Zhang et al., "Kinetic pathways towards mass production of single crystalline stanene on topological insulator substrates," Nanoscale, vol. 10, no. 40, pp. 18988-18994, Oct. 2018, doi: 10.1039/c8nr05815f.

[27] J. Li et al., "Anisotropic Electronic Structure and Interfacial Chemical Reaction of Stanene/Bi ${ }_{2} \mathrm{Te}_{3}$," J. Phys. Chem. C, vol. 124, no. 8, pp. 4917-4924, Feb. 2020, doi: 10.1021/acs.jpcc.0c00139.

[28] Y. Zang et al., "Realizing an Epitaxial Decorated Stanene with an Insulating Bandgap," Adv. Funct. Mater., vol. 28, no. 35, p. 1802723, 2018, doi: 10.1002/adfm.201802723.

[29] M. Liao et al., "Superconductivity in few-layer stanene," Nat. Phys., vol. 14, no. 4, pp. 344-348, Apr. 2018, doi: 10.1038/s41567-017-0031-6. 
[30] J. Yuhara et al., "Large area planar stanene epitaxially grown on $\operatorname{Ag}\left(\begin{array}{lll}1 & 1 & 1\end{array}\right)$," 2D Mater., vol. 5, no. 2, p. 025002, Jan. 2018, doi: 10.1088/2053-1583/aa9ea0.

[31] M. Maniraj et al., "A case study for the formation of stanene on a metal surface," Commun. Phys., vol. 2, no. 1, p. 12, Dec. 2019, doi: 10.1038/s42005-0190111-2.

[32] Y.-H. Song et al., "High-buckled $\sqrt{3} \times \sqrt{3}$ stanene with topologically nontrivial energy gap," p. 14, 2017.

[33] J. Deng et al., "Epitaxial growth of ultraflat stanene with topological band inversion," Nat. Mater., vol. 17, no. 12, pp. 1081-1086, Dec. 2018, doi: 10.1038/s41563-018-0203-5.

[34] S. Saxena, R. P. Chaudhary, and S. Shukla, "Stanene: Atomically Thick Freestanding Layer of 2D Hexagonal Tin,” Sci. Rep., vol. 6, no. 1, p. 31073, Nov. 2016, doi: $10.1038 /$ srep31073.

[35] Y. Guo et al., "Interfacial properties of stanene-metal contacts," 2D Mater., vol. 3, no. 3, p. 035020, Sep. 2016, doi: 10.1088/2053-1583/3/3/035020.

[36] J. Gao, G. Zhang, and Y.-W. Zhang, "Exploring Ag(111) Substrate for Epitaxially Growing Monolayer Stanene: A First-Principles Study," Sci. Rep., vol. 6, no. 1, p. 29107, Sep. 2016, doi: 10.1038/srep29107.

[37] L. Tao et al., "Silicene field-effect transistors operating at room temperature," Nat. Nanotechnol., vol. 10, no. 3, pp. 227-231, Mar. 2015, doi: 10.1038/nnano.2014.325.

[38] D.-A. Luh, C.-H. Wang, and Y.-W. Yang, "Growth of thin Sn films on $\mathrm{Ag}(111)$ studied with low-energy electron diffraction and X-ray photoelectron spectroscopy," Thin Solid Films, vol. 682, pp. 44-49, Jul. 2019, doi: 10.1016/j.tsf.2019.04.047.

[39] S. Nigam, S. Gupta, D. Banyai, R. Pandey, and C. Majumder, "Evidence of a graphene-like $\mathrm{Sn}$-sheet on a $\mathrm{Au}(111)$ substrate: electronic structure and transport properties from first principles calculations," Phys. Chem. Chem. Phys., vol. 17, no. 10, pp. 6705-6712, 2015, doi: 10.1039/C4CP04861J. 
[40] Y. Liu et al., "Realization of Strained Stanene by Interface Engineering," $J$. Phys. Chem. Lett., vol. 10, no. 7, pp. 1558-1565, Apr. 2019, doi:

10.1021/acs.jpclett.9b00348.

[41] M. Maniraj et al., "Structure and electronic properties of the $(3 \times 3) \mathrm{R} 30$ 。 SnA u 2 / Au ( 111 ) surface alloy," Phys. Rev. B, vol. 98, no. 20, p. 205419, Nov. 2018, doi: 10.1103/PhysRevB.98.205419.

[42] J. Yuhara and Y. Shichida, "Epitaxial growth of two-dimensional Pb and $\mathrm{Sn}$ films on Al(111)," Thin Solid Films, vol. 616, pp. 618-623, Oct. 2016, doi: 10.1016/j.tsf.2016.09.028.

[43] M. Araidai, M. Kurosawa, A. Ohta, and K. Shiraishi, "First-principles study on adsorption structure and electronic state of stanene on alpha-alumina surface," Jpn. J. Appl. Phys., vol. 56, no. 9, p. 095701, Sep. 2017, doi: 10.7567/JJAP.56.095701.

[44] H. Wang, S. T. Pi, J. Kim, Z. Wang, H. H. Fu, and R. Q. Wu, "Possibility of realizing quantum spin Hall effect at room temperature in stanene / A $12 \mathrm{O} 3$ ( 0001 )," Phys. Rev. B, vol. 94, no. 3, p. 035112, Jul. 2016, doi: 10.1103/PhysRevB.94.035112.

[45] S. Glass et al., "Triangular Spin-Orbit-Coupled Lattice with Strong Coulomb Correlations: Sn Atoms on a SiC(0001) Substrate," Phys. Rev. Lett., vol. 114, no. 24, p. 247602, Jun. 2015, doi: 10.1103/PhysRevLett.114.247602.

[46] F. Matusalem, F. Bechstedt, M. Marques, and L. K. Teles, "Quantum spin Hall phase in stanene-derived overlayers on passivated SiC substrates," Phys. Rev. B, vol. 94, no. 24, p. 241403, Dec. 2016, doi: 10.1103/PhysRevB.94.241403.

[47] F. Matusalem, D. S. Koda, F. Bechstedt, M. Marques, and L. K. Teles, "Deposition of topological silicene, germanene and stanene on graphene-covered $\mathrm{SiC}$ substrates," Sci. Rep., vol. 7, no. 1, p. 15700, Dec. 2017, doi: 10.1038/s41598-01715610-3.

[48] D. Di Sante et al., "Towards topological quasifreestanding stanene via substrate engineering," Phys. Rev. B, vol. 99, no. 3, p. 035145, Jan. 2019, doi: 10.1103/PhysRevB.99.035145.

[49] N. Ferdous, M. S. Islam, J. Park, and A. Hashimoto, "Tunable electronic properties in stanene and two dimensional silicon-carbide heterobilayer: A first 
principles investigation," Aip Adv., vol. 9, no. 2, p. 025120, Feb. 2019, doi: 10.1063/1.5066029.

[50] P. Li, "Stanene on a $\mathrm{SiC}(0001)$ surface: a candidate for realizing quantum anomalous Hall effect," Phys. Chem. Chem. Phys., vol. 21, no. 21, pp. 11150-11157, Jun. 2019, doi: 10.1039/c9cp01509d.

[51] R. P. Chaudhary, S. Saxena, and S. Shukla, "Optical properties of stanene," Nanotechnology, vol. 27, no. 49, p. 495701, Dec. 2016, doi: 10.1088/09574484/27/49/495701.

[52] D. Chiappe et al., "Two-Dimensional Si Nanosheets with Local Hexagonal Structure on a MoS 2 Surface," Adv. Mater., vol. 26, no. 13, pp. 2096-2101, Apr. 2014, doi: 10.1002/adma.201304783.

[53] R. van Bremen, Q. Yao, S. Banerjee, D. Cakir, N. Oncel, and H. J. W. Zandvliet, "Intercalation of Si between $\mathrm{MoS}_{2}$ layers," Beilstein J. Nanotechnol., vol. 8, pp. 1952-1960, Sep. 2017, doi: 10.3762/bjnano.8.196.

[54] L. Zhang et al., "Structural and Electronic Properties of Germanene on MoS 2," Phys. Rev. Lett., vol. 116, no. 25, p. 256804, Jun. 2016, doi: 10.1103/PhysRevLett.116.256804.

[55] D. Liang et al., "Tunable band gaps in stanene/MoS2 heterostructures," J. Mater. Sci., vol. 52, no. 10, pp. 5799-5806, May 2017, doi: 10.1007/s10853-0170817-z.

[56] W. Xiong et al., "Band engineering of the MoS2/stanene heterostructure: strain and electrostatic gating," Nanotechnology, vol. 28, no. 19, p. 195702, May 2017, doi: 10.1088/1361-6528/aa68d8.

[57] C.-C. Ren, Y. Feng, S.-F. Zhang, C.-W. Zhang, and P.-J. Wang, "The electronic properties of the stanene/ $\mathrm{MoS}_{2}$ heterostructure under strain," RSC Adv., vol. 7, no. 15, pp. 9176-9181, 2017, doi: 10.1039/C6RA26169H.

[58] K.-C. Chen, "Multi-layer elemental 2D materials: antimonene, germanene and stanene grown directly on molybdenum disulfides," Semicond Sci Technol, p. 8, 2019.

[59] M. Wang, L. Liu, C.-C. Liu, and Y. Yao, "van der Waals heterostructures of germanene, stanene, and silicene with hexagonal boron nitride and their topological 
domain walls," Phys. Rev. B, vol. 93, no. 15, p. 155412, Apr. 2016, doi: 10.1103/PhysRevB.93.155412.

[60] A. I. Khan, T. Chakraborty, N. Acharjee, and S. Subrina, "Stanene-hexagonal boron nitride heterobilayer: Structure and characterization of electronic property," Sci. Rep., vol. 7, no. 1, p. 16347, Dec. 2017, doi: 10.1038/s41598-017-16650-5.

[61] C. Yelgel, "Tunable electronic properties of van der Waals heterostructures composed of stanene adsorbed on two-dimensional, graphene-like nitrides," J. Appl. Phys., vol. 125, no. 15, p. 155301, Apr. 2019, doi: 10.1063/1.5096160.

[62] Y. Fang et al., "Quantum Spin Hall States in Stanene/Ge(111)," Sci. Rep., vol. 5, no. 1, p. 14196, Nov. 2015, doi: 10.1038/srep14196.

[63] L. Wu et al., "Structural and electronic properties of two-dimensional stanene and graphene heterostructure," Nanoscale Res. Lett., vol. 11, no. 1, p. 525, Dec. 2016, doi: 10.1186/s11671-016-1731-z.

[64] M. E. Bachra, H. Zaari, A. Benyoussef, A. E. Kenz, and A. G. E. Hachimi, "First-Principles Calculations of van der Waals and Spin Orbit Effects on the TwoDimensional Topological Insulator Stanene and Stanene on Ge(111) Substrate," J. Supercond. Nov. Magn., vol. 31, no. 8, pp. 2579-2588, Aug. 2018, doi: 10.1007/s10948-017-4503-9.

[65] J. M. Carpinelli, H. H. Weitering, M. Bartkowiak, R. Stumpf, and E. W. Plummer, "Surface Charge Ordering Transition: a Phase of SnGe(111)," Phys. Rev. Lett., vol. 79, no. 15, p. 4, 1997.

[66] A. Tejeda et al., "Structural Origin of the Sn 4 d Core Level Line Shape in Sn / Ge ( 111$)-(3 \times 3)$," Phys. Rev. Lett., vol. 100, no. 2, p. 026103, Jan. 2008, doi: 10.1103/PhysRevLett.100.026103.

[67] X. Chen et al., "Electronic structure and optical properties of graphene/stanene heterobilayer," Phys. Chem. Chem. Phys., vol. 18, no. 24, pp. 16302-16309, 2016, doi: 10.1039/C6CP02424F.

[68] F. F. Yun, D. L. Cortie, and X. L. Wang, "Tuning the electronic structure in stanene/graphene bilayers using strain and gas adsorption," Phys. Chem. Chem. Phys., vol. 19, no. 37, pp. 25574-25581, Oct. 2017, doi: 10.1039/c7cp03678g. 
[69] C. Mondal, S. Kumar, and B. Pathak, "Topologically protected hybrid states in graphene-stanene-graphene heterojunctions," J. Mater. Chem. C, vol. 6, no. 8, pp. 1920-1925, Feb. 2018, doi: 10.1039/c7tc05212j.

[70] M. Noshin, A. I. Khan, and S. Subrina, "Thermal transport characterization of stanene/silicene heterobilayer and stanene bilayer nanostructures," Nanotechnology, vol. 29, no. 18, p. 185706, May 2018, doi: 10.1088/1361-6528/aaaf17.

[71] M. Barhoumi, K. Lazaar, and M. Said, "DFT study of the electronic and vibrational properties of silicene/stanene heterobilayer," Phys. E-Low-Dimens. Syst. Nanostructures, vol. 111, pp. 127-129, Jul. 2019, doi: 10.1016/j.physe.2019.03.009.

[72] Y. Ding and Y. Wang, "Quasi-Free-Standing Features of Stanene/Stanane on InSe and GaTe Nanosheets: A Computational Study," J. Phys. Chem. C, vol. 119, no. 49, pp. 27848-27854, Dec. 2015, doi: 10.1021/acs.jpcc.5b08946.

[73] H. Zhang, T. Zhou, J. Zhang, B. Zhao, Y. Yao, and Z. Yang, "Quantum anomalous Hall effect in stanene on a nonmagnetic substrate," Phys. Rev. B, vol. 94, no. 23, p. 235409, Dec. 2016, doi: 10.1103/PhysRevB.94.235409.

[74] H. Zhang, Z. Wang, and X. Xu, "Room temperature quantum spin Hall insulator: Functionalized stanene on layered PbI2 substrate," Appl. Phys. Lett., vol. 111, no. 7, p. 072105, Aug. 2017, doi: 10.1063/1.4985643.

[75] Z. Ni, E. Minamitani, Y. Ando, and S. Watanabe, "Germanene and stanene on two-dimensional substrates: Dirac cone and Z 2 invariant," Phys. Rev. B, vol. 96, no. 7, p. 075427, Aug. 2017, doi: 10.1103/PhysRevB.96.075427.

[76] X. Chen, Y. Li, J. Tang, L. Wu, D. Liang, and R. Zhang, "First-principles study on electronic properties of stanene/WS 2 monolayer," Mod. Phys. Lett. B, vol. 31, no. 29, p. 1750271, Oct. 2017, doi: 10.1142/S0217984917502712.

[77] H. Cao, Z. Zhou, X. Zhou, and J. Cao, "Tunable electronic properties and optical properties of novel stanene/ZnO heterostructure: First-principles calculation," Comput. Mater. Sci., vol. 139, pp. 179-184, Nov. 2017, doi: 10.1016/j.commatsci.2017.08.001.

[78] B. Chakraborty, M. M. Borgohain, and N. C. Adhikary, "Structural and electronic properties of Stanene-BeO heterobilayer," Mater. Res. Express, vol. 7, no. 1, p. 015029, Jan. 2020, doi: 10.1088/2053-1591/ab6091. 



\section{CHAPTER 3}

\section{Epitaxial Sn on silicene terminated}

\section{$\mathrm{ZrB}_{2}$}

\subsection{Introduction}

The field of two-dimensional (2D) materials has significantly expanded, since the discovery of graphene [1]. In particular, considerable efforts have been devoted to the synthesis of group IV 2D materials, such as silicene [2][3][4] germanene [5][6][7] and stanene [8][9][10]. These elemental 2D materials are the most chemically simple among the entire 2D library, and exhibit several promising properties that outperform those of graphene [11]. One of the examples is their 2D topological insulator phase important for the observation of the quantum spin Hall effect. Such an exotic electronic phase is derived from the energy gap opening at the Dirac points due to the buckled atomic structure combined with robust spin-orbit coupling. A very recent development in the field is to create 2D alloys, as inspired by the bulk alloys of group IV elements in enhancing the performance of silicon-based electronic devices [12][13]. Group IV 2D alloys, in single- or few-layer form, are advantageous for potentially offering new properties and applications unattainable in their parent, elemental counterparts. Specific characteristics that can be tuned or modified by forming such alloys include the electronic band structure (which governs important characteristics such as the Dirac point and band gap), carrier mobility, hybridization with substrate bands, and the spinorbit coupling strength as well as lattice parameters and associated strain effects [14][15][16][17]. 
To study the feasibility of fabricating such $2 \mathrm{D}$ alloy systems and to determine their electronic properties, we investigate in this work the interaction of Sn atoms with silicene grown on a $\mathrm{ZrB}_{2}$ substrate. The sample fabrication consisted of two parts: (i) In-situ deposition of $\mathrm{Sn}$ at room temperature, and (ii) annealing at temperatures up to $650^{\circ} \mathrm{C}$. During the experiment we discovered three distinct structures, by using low energy electron diffraction (LEED), scanning tunnelling microscopy (STM) and spectroscopy (STS), and photoelectron spectroscopy (PES). After a brief description of the experimental methods and the characteristics of pristine silicene on $\mathrm{ZrB}_{2}$, these structures will be discussed in separate sections that are identified with the temperature range in which the structures are formed.

\subsection{Methods}

\subsubsection{Silicene-terminated $\mathrm{ZrB}_{2}(0001)$ thin films on $\mathrm{Si}(111)$}

$\mathrm{ZrB}_{2}$ thin films were grown on $\mathrm{Si}(111)$ substrates by ultra-high vacuum chemical vapor epitaxy (UHV-CVE), which is described elsewhere[18]. During the growth at elevated temperature, silicon atoms originating from the substrate segregate to the surface of $\mathrm{ZrB}_{2}$ thin film. Upon cooling down to temperatures below $650^{\circ} \mathrm{C}$, a monolayer of silicon atoms crystallizes in a honeycomb lattice - silicene - terminating the whole $\mathrm{ZrB}_{2}(0001)$ thin film surface. The epitaxial relation between the silicene layer and the $\mathrm{ZrB}_{2}$ substrate is such that the $(\sqrt{3} \times \sqrt{3})$ unit cell of the former matches the $(2 \times 2)$ unit cell of the latter. Taking it out from UHV to ambient conditions causes an immediate oxidation of the $\mathrm{Si}$ layer and $\mathrm{ZrB}_{2}$ thin film surface beneath. However, silicene can be easily recovered by annealing at $800^{\circ} \mathrm{C}$ in $\mathrm{UHV}$. Each time an experiment was performed using a sample brought from ambient conditions, silicene was reproduced by annealing at $650^{\circ} \mathrm{C}$ for $10 \mathrm{~h}$ and subsequent annealing at $800^{\circ} \mathrm{C}$ for $1 \mathrm{~h}$. This treatment removes native oxides and recovers silicene. The quality of the recovered sample surface was then checked by LEED and STM as well as PES.

\subsubsection{Sn deposition}

Sn was deposited on a clean silicene-terminated sample, held at room temperature. The source was a commercial effusion cell. Its rate was calibrated using a clean $\mathrm{Si}(111)$ surface and the well-known transition of the Sn-derived surface reconstruction from $\sqrt{ } 3$ $x \sqrt{3}$ to $2 \sqrt{3} \times 2 \sqrt{3}$, upon deposition of an amount equivalent to $1 / 3 \mathrm{ML}$ of $\mathrm{Si}(1 \mathrm{ML}=$ $7.8 \times 10^{14} \mathrm{at} / \mathrm{cm}^{2}$, the areal density of atoms along the perfect $\mathrm{Si}(111)$ surface plane[19]) and subsequent annealing at $650^{\circ} \mathrm{C}$. Based on that calibration, an amount corresponding 
to $1 \mathrm{ML}$ of $\mathrm{Sn}\left(1 \mathrm{ML}=10.7 \times 10^{14} \mathrm{at} / \mathrm{cm}^{2}\right.$ areal density of $\mathrm{Sn}$ atoms in free-standing stanene [20]) was deposited, leading to the deposition rate of approx. $1 \mathrm{ML}$ of Sn per 5 $\min$.

\subsubsection{Measurement methods}

Several experiments were performed using a few distinct setups, all offering UHV conditions with the base pressure not exceeding $5.0 \times 10^{-9} \mathrm{mbar}$. Prior to those in-situ experiments, the sample was cleaned with the above-mentioned procedure. The measurements were performed with STM systems at JAIST and at National University of Singapore (NUS). The system at JAIST consists of a load lock and a single UHV chamber (base pressure of $1.0 \times 10^{-10} \mathrm{mbar}$ ) with preparation and analysis parts. In the preparation part, the Sn source and a LEED system are located. The STM system was an Omicron VR-STM. STM measurements were performed at room temperature, in constant-current mode and with chemically etched Pt/Ir tips. Additional STM and STS measurements were done at $77 \mathrm{~K}$, using an Omicron low temperature (LT-)STM system at NUS. The system consisted of a load lock, a preparation chamber with a Sn source and an analysis chamber (base pressure $2.0 \times 10^{-10} \mathrm{mbar}$ ). The STM measurements were performed in constant current mode using chemically etched Pt/Ir tips, while the STS spectra ( $d I / d V$ curves) were recorded with a lock-in amplifier by superimposing an ac voltage $(30 \mathrm{mV}, 963 \mathrm{~Hz})$ on the given dc tip bias relative to the sample. PES spectra were taken using the ANTARES beamline at the SOLEIL synchrotron facility, France. The ANTARES end station consists of 4 interconnected chambers: a load-lock, a preparation chamber, a storage chamber and an analysis chamber. The preparation chamber (base pressure $2.0 \times 10^{-10} \mathrm{mbar}$ ) is equipped with a $\mathrm{Sn}$ source (an effusion cell) and a LEED system. The sample holder allows for direct current heating. The analysis system has access to the synchrotron x-rays and contains a NanoARPES Scienta R4000 detector setup.

\subsection{Results and discussion}

\subsubsection{Silicene on $\mathrm{ZrB}_{2}$}

Silicene on $\mathrm{ZrB}_{2}$ has been studied extensively in recent years and is now a well understood system [21][22][23]. STM and LEED data acquired from such a pristine

sample are shown in Fig. 3.1. The STM images show several characteristic features. Silicon from the $\mathrm{Si}(111)$ substrate segregates spontaneously to the surface and forms a 
honeycomb monolayer aligned with the $2 \times 2$ unit cell of the underlying $\mathrm{ZrB}_{2}$ substrate. This corresponds to the $\sqrt{ } 3 \times \sqrt{ } 3$ unit cell of silicene, with $1 \times 1$ lattice constant of $3.65 \AA$.

The former unit cell is manifested as protrusions in atomic resolution STM images, as highlighted by the blue diamond in Fig. 3.1a, in agreement with the "planar-like" buckling structure of silicene on $\mathrm{ZrB}_{2}$ that features a single protruding $\mathrm{Si}$ atom in the unit cell [23]. Large-scale STM images show that the surface and hence the silicene lattice spontaneously develops as "stripes". The same stripes manifest themselves in the LEED image, as a splitting of the $\sqrt{ } 3$ spots into groups of six fractional spots (marked in orange in Fig. 3.1b [24]. It has been proposed that the driving force behind the formation of the stripe pattern is associated with a phonon instability characterized by a zero-frequency mode reaching a critical point of instability [25]. That instability is related to the epitaxial strain imposed onto silicene by the $\mathrm{ZrB}_{2}$ thin film surface which has a lattice mismatch of about $5 \%$ to the predicted value of hypothetical, free-standing silicene. Another important feature of silicene on $\mathrm{ZrB}_{2}$ is related to its $\mathrm{Si} 2 p$ core level spectrum (see Fig. 3.2) that is composed of three sets of Si $2 p$ doublets originating from three distinct chemical species of $\mathrm{Si}$, as previously reported [23]. 

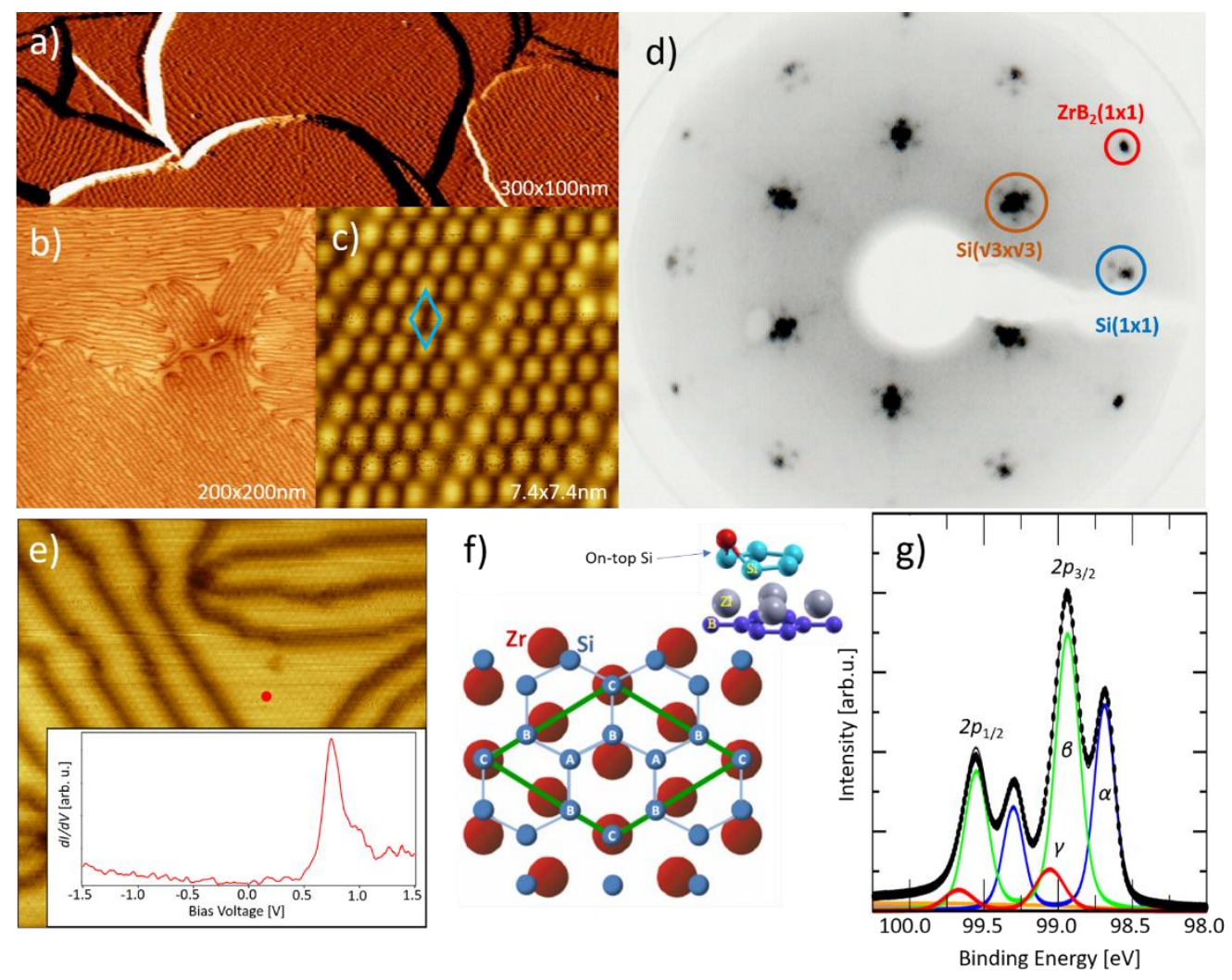

Fig. 3.1: Various characteristics of a clean silicene terminated $\mathrm{ZrB}_{2}$ sample. (a-c) STM measurements of a) $\mathrm{ZrB}_{2}$ terraces covered with silicene. b) Stripe domains, corresponding to the native surface reconstruction of the system.( reproduced from [26] ) c) Atomically resolved surface structure, with the silicene $\sqrt{3} \times \sqrt{3}$ unit cell (blue diamond) matching the $2 \times 2$ supercell of $\mathrm{ZrB}_{2}$. d) LEED pattern of the clean sample with indications of each set of spots that appear. $\mathrm{Si}$ spots match the half-order fractional spots of the $\mathrm{ZrB}_{2}(0001)-(2 \times 2)$ reconstruction. Note that the $\mathrm{Si} \sqrt{3} \times \sqrt{3}$ spots are split into fractional spots, which is caused by the existence of the stripes. e) STS measurement, performed at the position of the red dot in the corresponding STM image. f) Atomic model of the surface showing the arrangement of Si atoms on the surface, with respect to the $\mathrm{ZrB}_{2}$ substrate (Adapted from [25][27] ). DFT calculations gave insight about the out-ofplane displacements of $\mathrm{Si}$ atoms, showing that the most energetically favorable arrangement is planar-like with one $\mathrm{Si}$ atom, located directly above a $\mathrm{Zr}$ atom, standing out. One can recognize 3 distinct sites which contribute to g) the $\mathrm{Si} 2 p$ core level spectrum, measured by PES. The different intensity of different $\mathrm{Si} 2 p$ doublets, labeled $\alpha, \beta, \gamma$, correspond to the ratio of the occupation numbers of atoms on each different site in the unit cell, labeled a,b, c. Reproduced from [23] 


\subsection{2 $250-350^{\circ} \mathrm{C}$ Regime}

Approximately $1 \mathrm{ML}$ of $\mathrm{Sn}$ was deposited on the silicene-terminated surface. Then the sample was annealed stepwise in increments of $50^{\circ} \mathrm{C}$, starting from $200^{\circ} \mathrm{C}$, which was the lower measurement limit of the pyrometer. Between $250^{\circ} \mathrm{C}$ and $350^{\circ} \mathrm{C}$, a new LEED pattern appeared (inset in Fig. 3, 2a). The structure was then investigated with STM. As evidenced by the large-scale STM image in Fig. 2, three distinct structures become visible on the sample surface after the deposition of $1 \mathrm{ML} \mathrm{Sn}$ and annealing at $325^{\circ} \mathrm{C}$.

Structure $\alpha$ exhibits a quasi-triangular pattern, similar to the previously observed metallic silicon-based layer on silicene/ $/ \mathrm{ZB}_{2}$. In that experiment around $0.5 \mathrm{ML}$ of $\mathrm{Si}$ atoms were deposited on the sample at elevated temperature within $250^{\circ} \mathrm{C}-350^{\circ} \mathrm{C}$ range [28] (see top panel of Fig. 3.3). Since the latter consists of an additional layer of $\mathrm{Si}$ atoms deposited onto a silicene layer, in the following we will refer to this system as "Si bilayer" for brevity. Its most readily observed characteristic is the LEED pattern, in which the $\sqrt{ } 3 \times \sqrt{3}$ Si spots change their shape, while the Si $1 \times 1$ spots double (Fig. 3.3c). Atomic-scale STM measurements indeed verify such a resemblance, as both structures have the same lattice constant of $6.52 \AA$ (Fig. 3.3a and 3.3e). Further confirmation is obtained by STS measurements, revealing a metallic $d I / d V$ curve when the measurement is recorded on the quasi-triangular structure, as expected for the Si bilayer.

In contrast to structure $\alpha$, it was not possible to obtain STM images of structure $\beta$ in atomic resolution. The $d I / d V$ curve reveals its metallic nature. It is supposedly formed by disordered $\mathrm{Sn}$. As we will elaborate later using the $\mathrm{Zr} 3 d$ core level PES spectra (Fig. 3.4), the disordered $\mathrm{Sn}$ is most probably in direct contact with the $\mathrm{Zr}$ atoms of the $\mathrm{ZrB}_{2}$ surface. This explains the formation of additional Si-based structures on the surface, e.g. the above-mentioned Si bilayer, which comprises $\mathrm{Si}$ atoms ejected from the initial silicene layer.

Similar to structure $\alpha$, structure $\gamma$ strongly resembles the previous structure that resulted from deposition of an additionally small $(<0.1 \mathrm{ML})$ amount of $\mathrm{Si}$ atoms on the silicene $/ \mathrm{ZrB}_{2}$ surface [24]. That small amount of extra material "seals" the stripes and forms a meta-stable single-domain silicene lattice on the surface, with a slightly different lattice constant of $6.47 \AA$. Similar to structure $\beta$, the $d I / d V$ curve shows a local maximum on the positive bias (unoccupied states) side. The location of the maxima in the $d I / d V$ curves of both structure $\beta$ and $\gamma$ indicates that they originate from the substrate [21]. A strong contribution from the substrate suggests that both structures are of ML 
thickness. It is also consistent with the lack of such a maximum in the case of structure $\alpha$, which is known to be a multilayer, hence the substrate signal is significantly lower.
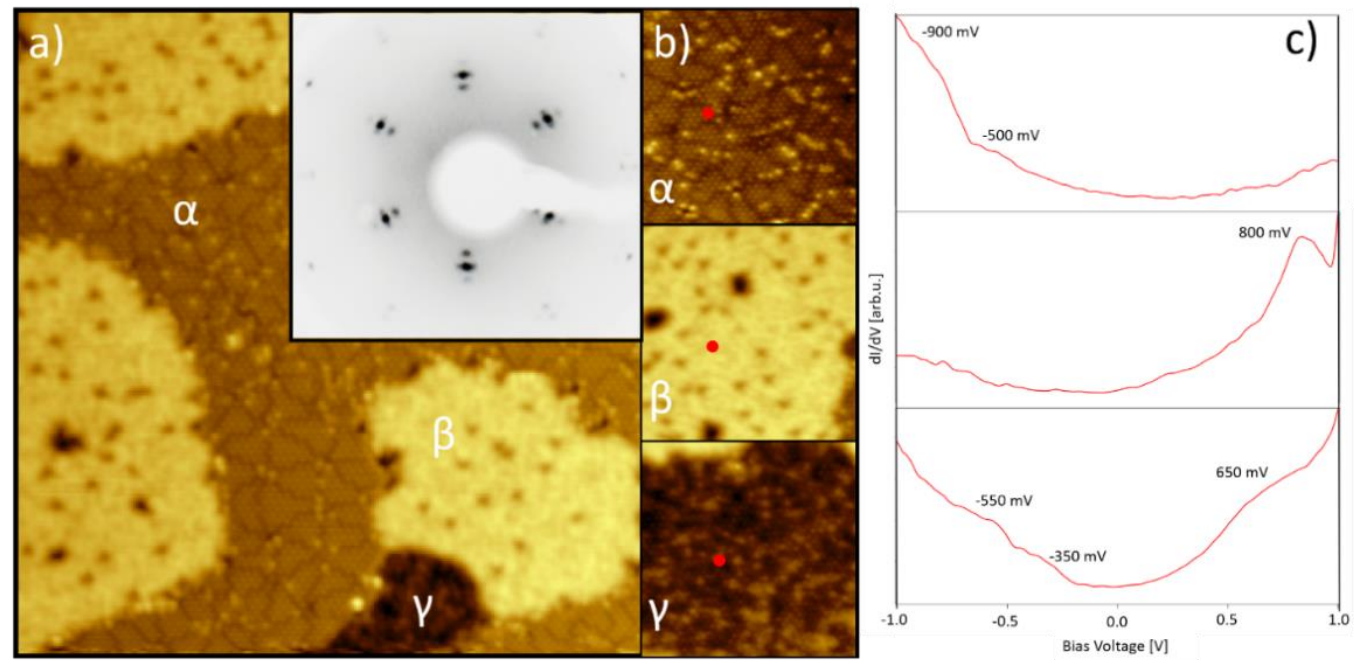

Fig. 3.2: LEED, STM and STS measured after deposition of approximately 1 ML of Sn and annealing at $325^{\circ} \mathrm{C}$. a) Large scan area $\left(50 \times 50 \mathrm{~nm}^{2}, V_{\text {Bias }}=0.8 \mathrm{~V}, I_{s t p}=50 \mathrm{pA}, T=77 \mathrm{~K}\right) \mathrm{STM}$ image with 3 structures coexisting on the surface of the sample. b) Small area $\left(20 \times 20 \mathrm{~nm}^{2}\right)$ STM images of each structure in a). c) Corresponding $d I / d V$ curves obtained by STS measurements taken at points indicated by the red dots in $b$ ).

The strong resemblance to the reports on Si deposition on this sample [28], prompted us to look for more similarities. By varying the amount of deposited Sn, we observed an evolution of the LEED pattern that mimics the one obtained in $\mathrm{Si}$ deposition experiments. The similarity is very clearly visible in Fig. 3.3, where a comparison between results acquired after Si deposition and Sn deposition is made. Essentially the same LEED pattern was obtained in our case by depositing a small amount of Sn. The change of the $\sqrt{3} \times \sqrt{3}$ Si feature from its original arrangement of fractional spots to a single spot corresponds to a single Si domain on the surface. The pattern obtained by deposition of $1 \mathrm{ML}$ of $\mathrm{Sn}$ also resembles the one reported in the $\mathrm{Si}$ deposition experiment. That pattern is associated with the Si bilayer. Interestingly, upon depositing more $\mathrm{Sn}$, there are additional features appearing near the center of the LEED picture. Their origin is so far unknown as no structures with corresponding periodicity have been 
detected in STM measurements. It has been reported that deposition of an additional amount of Si up to 9 ML [28] does not change the LEED pattern presented in Fig. 3.3d. Therefore, we speculate that the new spots in Fig. 3h may come from a Sn-related structure, with a considerably large period $>1 \mathrm{~nm}$.
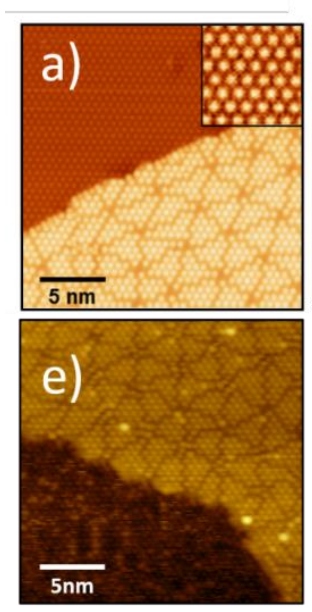
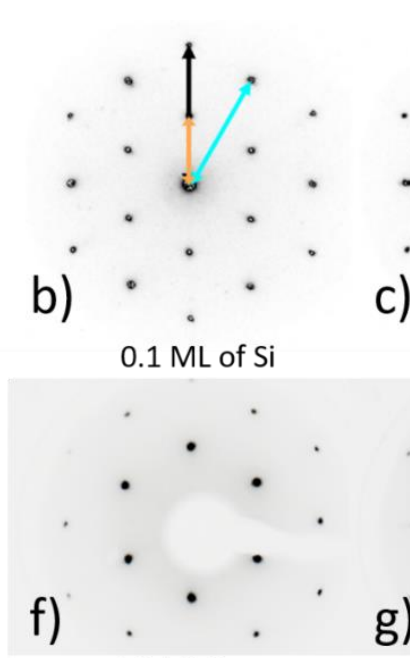

$0.2 \mathrm{MLSn}$

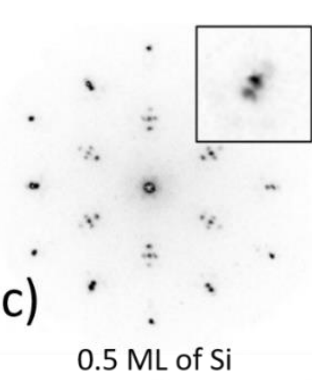

$1 \mathrm{ML}$ of $\mathrm{Sn}$ h)
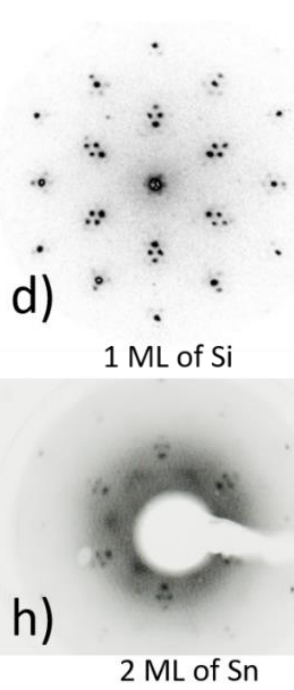

Fig. 3.3: STM and LEED comparison of Si (top, a-d) and Sn (bottom, e-h) deposition experiments, resulting in similar structures forming on top of the surface. a) and e) comparison between STM images of the structures obtained by depositing Si and Sn. While the triangular structures look the same, the lower terrace differs, probably as a result of mobile or disordered Sn atoms present on the surface. The $\mu$ LEED patterns b)-d) for the Si-deposition experiment match the experimental data obtained for Sn deposition f)-h). a)-d) were reproduced from Gill et al. [28]

The similarities between the results of the two experiments suggest that the origin of the three structures can be explained by partial removal of the original Si lattice upon $\mathrm{Sn}$ deposition and annealing. Sn atoms are then in direct contact with the outermost $\mathrm{Zr}$ atoms of the $\mathrm{ZrB}_{2}$ substrate, and the expelled $\mathrm{Si}$ atoms form a second layer on top of the silicene lattice, thus corresponding to the quasi triangular structure (structure $\alpha$ in Fig. 3.2a). Presumably, structure $\beta$ in Fig.3. 2a corresponds to a disordered Sn layer on $\mathrm{ZrB}_{2}$, possibly also containing $\mathrm{Si}$ atoms. This hypothesis is consistent with PES measurements 
(Fig. 3.4). One can observe an additional feature in the $\mathrm{Zr} 3 d$ core level spectrum. Similarly, a change in relative proportions between the different components in the $\mathrm{Si}$ $2 p$ spectrum suggest significant modification of the silicene structure. The overall shape most probably originates from (i) sealing stripes and forming a single-domain structure and (ii) contributions of the new chemical species of Si atoms that form the triangular pattern. Interestingly the PES spectra do not provide a direct indication of mixing between $\mathrm{Sn}$ and $\mathrm{Si}$. 

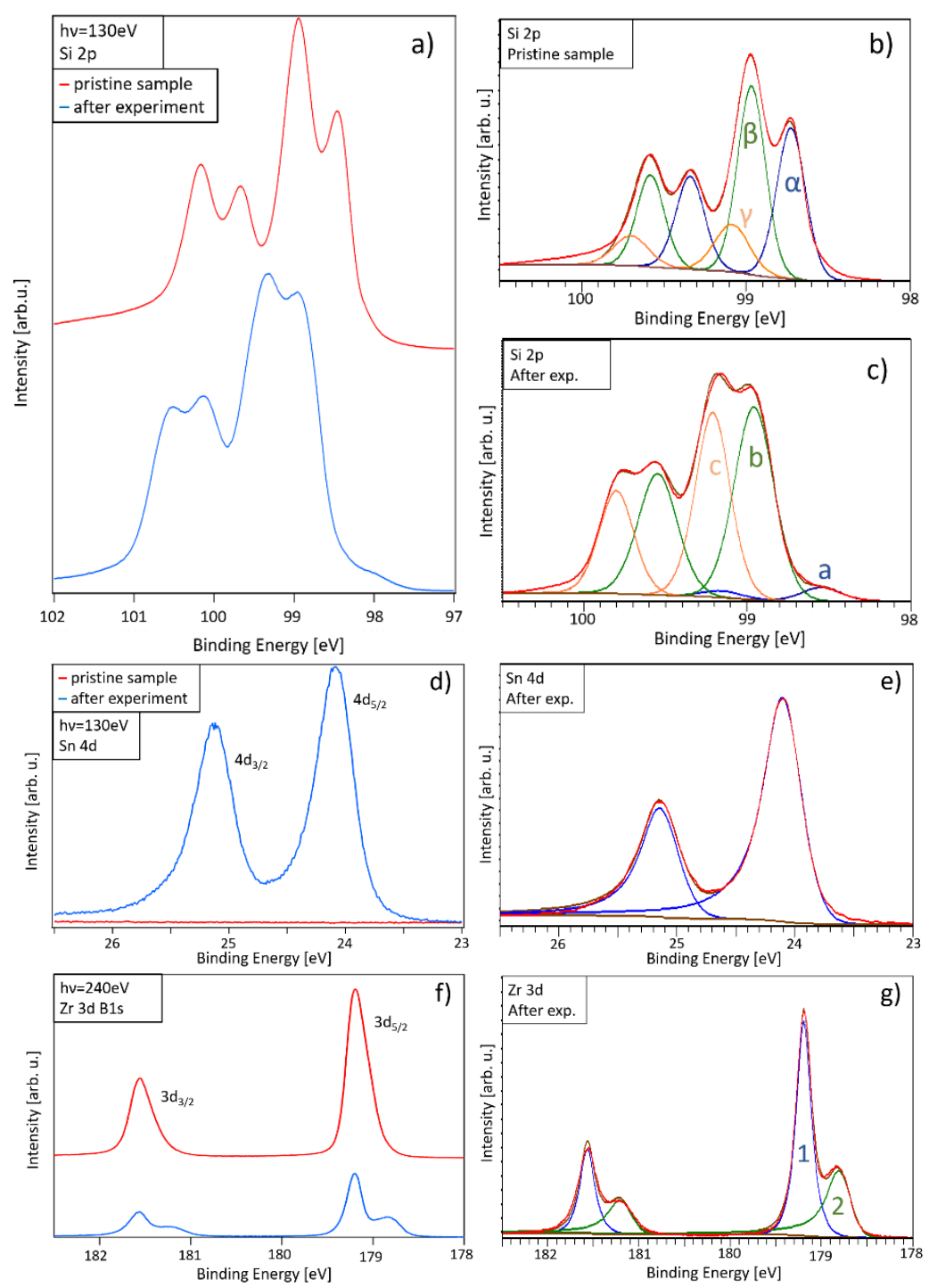

Fig. 3.4: PES core level spectra as well as peak fits of Si $2 p$ (a-c), Zr $3 d$ (d-e) and Sn $4 d$ (f-g) of a pristine sample and after deposition of approximately $1 \mathrm{ML}$ of $\mathrm{Sn}$ and annealing at $325^{\circ} \mathrm{C}$. In a) the main Si peaks shifted significantly towards higher binding energy. The clean sample data is consistent with the established fit (b) with 3 components [3][23]. The spectrum after the treatment has changed its shape significantly. It still can be fitted reasonably well with three components (c). A Sn peak appears after deposition and can be fitted with a single assymmetric doublet (d-e) The assymetry and FWHM of the new component of the $\mathrm{Zr} 3 d$ doublet (f-g) matches the parameters of Sn, which may suggest that the $\mathrm{Sn}$ and $\mathrm{Zr}$ atoms are in contact. 
The Si atoms which have been removed from the silicene layer appear to behave in the same manner as the additionally deposited $\mathrm{Si}$ atoms on clean surfaces - first they seal the stripes, which leads to formation of a single - domain surface, then, when a sufficient amount of $\mathrm{Si}$ is present, an additional layer of $\mathrm{Si}$ forms. That layer is metallic in nature and exhibits the characteristic triangular pattern.

Fig. 3.4 shows PES spectra of the pristine sample as well as after 1ML Sn deposition and annealing at $325^{\circ} \mathrm{C}$. It is seen from Fig. $4 \mathrm{~d}$ that a $\mathrm{Sn} 4 d$ core level doublet emerges upon the deposition, with its $4 d_{5 / 2}$ peak positioned at a binding energy (BE) of $24.1 \mathrm{eV}$. The $\mathrm{Zr} 3 d$ core level has its stronger $3 d_{5 / 2}$ peak maximum at $179.19 \mathrm{eV}$. A new component, shifted by $0.36 \mathrm{eV}$ with respect to the original peak, emerged after the experiment. The Si $2 p$ core level spectrum also changes considerably. The features are broadened, probably due to disorder introduced in structures that contain $\mathrm{Si}$ atoms. Around $98.5 \mathrm{eV}$ one can see a shoulder, which is an indication of the triangular structure of bilayer silicon.

To get an insight into the nature of the changes in the PES spectra, the $\mathrm{Si} 2 p, \mathrm{Zr} 3 d$ and Sn $4 d$ core levels were fitted. The Si $2 p$ core level spectrum of the clean sample was fitted (Fig. 3.4b) in accordance to the previously reported fitting procedure, using three components corresponding to three atomic species of Si (see Fig. $3.1 \mathrm{e}-\mathrm{f}$ ). The measured $2 p_{3 / 2}$ maxima are $98.73 \mathrm{eV}, 98.96 \mathrm{eV}$ and $99.09 \mathrm{eV}$ for peaks $\alpha, \beta$ and $\gamma$ respectively. In the Si $2 p$ spectrum one can notice a small tail at the higher binding energy side, most probably due to residual suboxides on the sample surface after the annealing treatment. After the Sn deposition and annealing, the Si $2 p$ core level changed significantly. Fig. $3 c$ shows again three components with their $2 p_{3 / 2}$ maxima at $98.55 \mathrm{eV}, 98.96 \mathrm{eV}$ and $99.21 \mathrm{eV}$ for peaks $\mathrm{a}, \mathrm{b}$, and $\mathrm{c}$ respectively. The matching positions of the $\mathrm{b}$ and $\beta$ components suggest the same origin of the two, which is the so-called near bridge site of $\mathrm{Si}$ atoms with respect to underlying $\mathrm{Zr}$ layer (see Fig. 3.1f). The other two components are shifted away from the central peak relative to the corresponding components in the clean sample. The relative ratio between the three after the experiment changed drastically, therefore new chemical species must contribute to the overall spectrum. We conclude that the presence of the silicon bilayer has altered the chemical environment of the underlying silicene lattice, such that the new peaks do not match their original position. We cannot, however, rule out the possiblity that $\mathrm{Sn}$ also has an influence on the chemical environment of $\mathrm{Si}$ atoms. More information about $\mathrm{Sn}$ is given by the two remaining fits, of $\mathrm{Sn} 4 d$ and $\mathrm{Zr} 3 d$ core levels (Fig. 3.4e, 3.4g). The 
Sn core level has been fitted with a single doublet. Apart from a slightly asymmetric shape, the peak has no additional discernable features. The assymetry might be an indication of metallic properties. This is in accordance with STS data, which also show metallic $d I / d V$ curve. The comparatively large width of the Sn $4 d$ peaks can be ascribed to inhomogeneous broadening and is, therefore, indicative of disorder. A similarly broad and asymmetric doublet in the $\mathrm{Zr} 3 d$ spectrum suggests that $\mathrm{Zr}$ atoms are in direct contact with $\mathrm{Sn}$ atoms, where disorder-related inhomogenous broadening affects both core-levels approximately equally and mainly determines their FWHM. Consequently, a part of $\mathrm{Si}$ atoms must have been removed from the silicene layer that initially completely covered the surface.

\subsection{3 $350-500^{\circ} \mathrm{C}$ Regime}

The Si bilayer structure disappears upon annealing at temperatures above $350^{\circ} \mathrm{C}$. Instead, other structures were discovered on the surface during the annealing procedure (Fig. 3.5). While these are the only structures that can be observed between $400-500^{\circ} \mathrm{C}$, the set of spots indicated by green arrows in the LEED pattern (Fig. 3.5c) is visible from directly after deposition until around $550^{\circ} \mathrm{C}$ when it disappears completely. A possible interpretation of the new spots are 2 hexagonal domains rotated by $15^{\circ}$ and $45^{\circ}$ with respect to $\mathrm{ZrB}_{2}$ lattice. The estimated lattice constant of such domains would be around $4.1 \AA$, which is different from both the expected lattice constants of the free-standing stanene ( $4.56 \AA$ ), and of the epitaxial silicene ( $3.65 \AA$ ). Unfortunately, in our STM measurements we were not able to find structures directly responsible for the pattern. Hence the origin of the LEED spots is still unknown. However, STM measurements did show new structures. Fig. 3.5 shows STM images of two kinds of atomically resolved structures. Although they both resemble single domain silicene with essentially the same lattice constant, the difference between the two is readily observable, and the border between the 2 structures is very sharp (see Fig. 3.5b). The clearly resolved islands have an appearance that is typical for alloys, exhibiting two distinct contrast levels for different atoms that may be attributed to $\mathrm{Sn}$ atoms incorporated in a Si lattice. This explanation seems to be feasible in the light of the report on Ge deposition on the same sample, where a very similar structure has been explained by Ge atoms replacing the Si atoms in the "on-top" site (marked as C in Fig. 3.1f). The distribution of $\mathrm{Ge}$ atoms was random and it created a mosaic-like $\mathrm{Si}_{6-\mathrm{x}} \mathrm{Ge}_{\mathrm{x}}$ alloy [29]. A somewhat similar structure has also been reported for the $\mathrm{Sn} / \mathrm{Si}(111)$ system, for sub-monolayer coverage [30][31]. It was the so-called $\gamma$-Sn or "mosaic 
phase" of the $\mathrm{Sn} / \mathrm{Si}(111)-\sqrt{ } 3 \times \sqrt{ } 3 \mathrm{R} 30^{\circ}$ reconstruction. However, that structure required higher temperature to form and lower coverage than the conditions in our experiment.
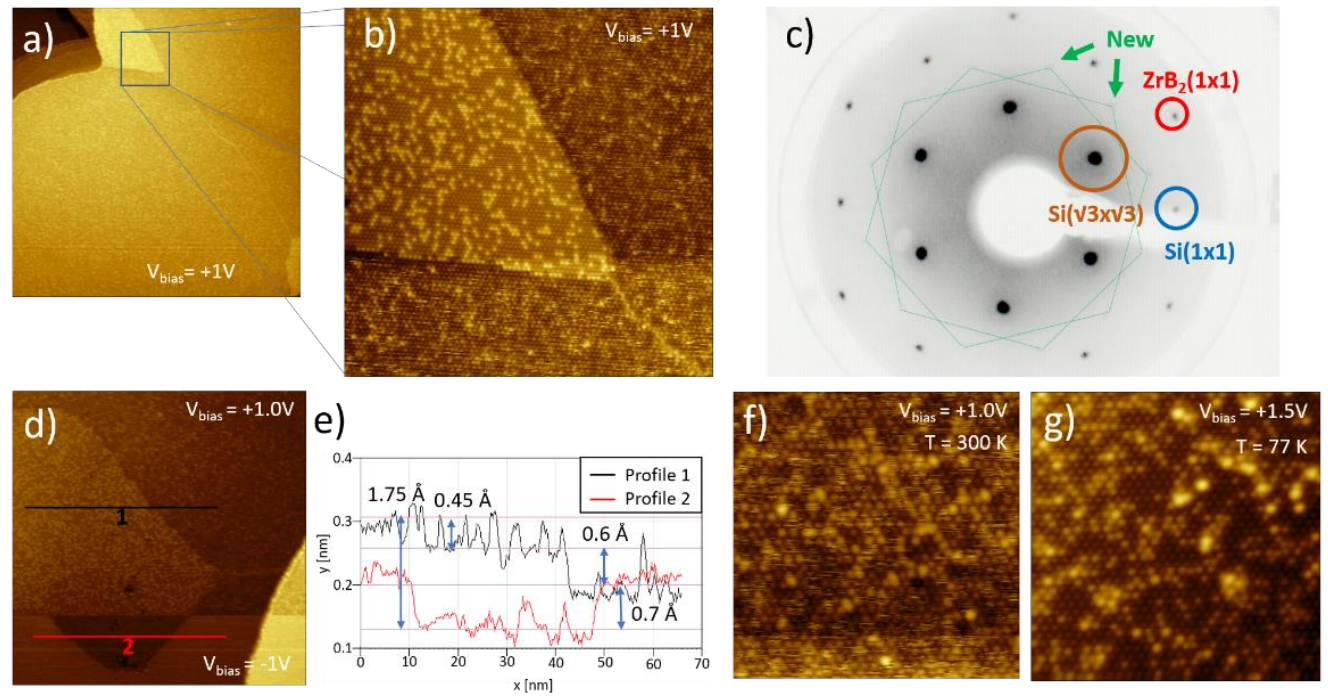

Fig. 3.5: STM images and LEED pattern of the structures observed in the $400-500^{\circ} \mathrm{C}$ temperature regime. Figures a)-b) show two visibly distinguishable structures with atomically sharp border (scan area: (a) $200 \times 200 \mathrm{~nm}^{2}$, (b) $55 \times 55 \mathrm{~nm}^{2}$ ). The top left, cone-like formation with clean, atomically resolved, alloy-like structure is surrounded by a blurry phase. c) LEED pattern, showing 12 weak spots (indicated with green arrows) and a halo around single $\sqrt{ } 3 \times \sqrt{ } 3$ Si spots. The 12 spots can be interpreted as 2 hexagonal patterns rotated by $30^{\circ}$ with respect to each other. d)-e) (scan area: $200 \times 200 \mathrm{~nm}^{2}$ ) The relative step height between the two structures (alloylike on the left and "blurry" one on the right of the image) is strongly bias dependent, and even changes sign depending on the bias. (f-g) The blurred structure becomes better resolved at lower temperatures, suggesting that thermally activated fluctuations of the positions of atoms are at the origin of the blurred appearance.

In the present case, similar Sn-Si alloy islands are surrounded by a "blurry" layer. It always remains blurry under any experimental conditions, despite having sharp atomic resolution on the alloy island (top left corner of Fig. 3.5b), an indication of a high-quality tip. This suggests the existence of mobile atoms on the surface, i.e. atoms that change 
their position on a timescale that is fast compared to the STM measurement, which could cause blurring of the image. It is not clear however what causes the sharp borders. The bias dependence of the STM images is very pronounced, with the relative apparent height between the two structures changing from alloy - like islands being higher at negative biases, to equal level at low voltages, to the blurry structure being higher at positive bias voltages (Fig. 3.6). The blurry structures are better resolved at lower temperatures (Fig. $3.5 \mathrm{f}-\mathrm{g}$ ) which confirms the statement regarding mobile atoms on the surface. Interestingly, the blurry phase also becomes better resolved at high negative voltages. One possible explanation could be the high DOS of the underlying surface with simultaneous low DOS of the adlayer, making the STM image insensitive to the on-top layer. The nature of the blurry structure might be similar to the well documented case of $\mathrm{Sn}$ on $\mathrm{Si}(111)$, where fast probe techniques like PES and LEED suggest the existence of a structure that is not visible in slow-probe techniques like STM [32].
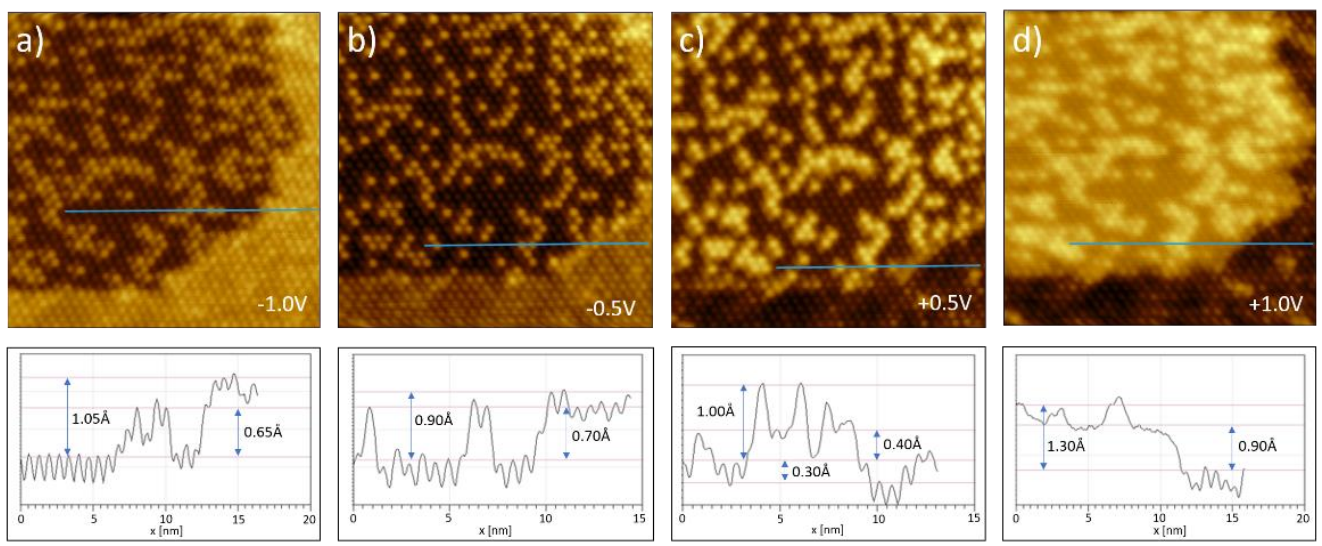

Fig. 3.6: Bias dependence of the relative step height between the two different structures observed on the surface (alloy - like on the left, surrounded by the blurred structure), determined from STM images recorded at $77 \mathrm{~K}$ and at different bias voltages. One can observe a strong change in relative apparent height between the two regions. 
Fig. 3.7 shows PES spectra of the sample after annealing in the temperature range between $400^{\circ} \mathrm{C}$ and $500^{\circ} \mathrm{C}$. A Sn $4 d$ core level doublet emerges upon deposition, with the stronger peak at a binding energy of $23.9 \mathrm{eV}$. The shape of the Si $2 p$ core level spectrum also changes considerably, it resembles now a bulk Si $2 p$ peak, suggesting that the majority of $\mathrm{Si}$ atoms reside in a similar chemical environment. The $\mathrm{Zr} 3 d$ peak has still the double component, which would suggest that there is still a significant amount of $\mathrm{Sn}$ in direct contact with the substrate surface layer.
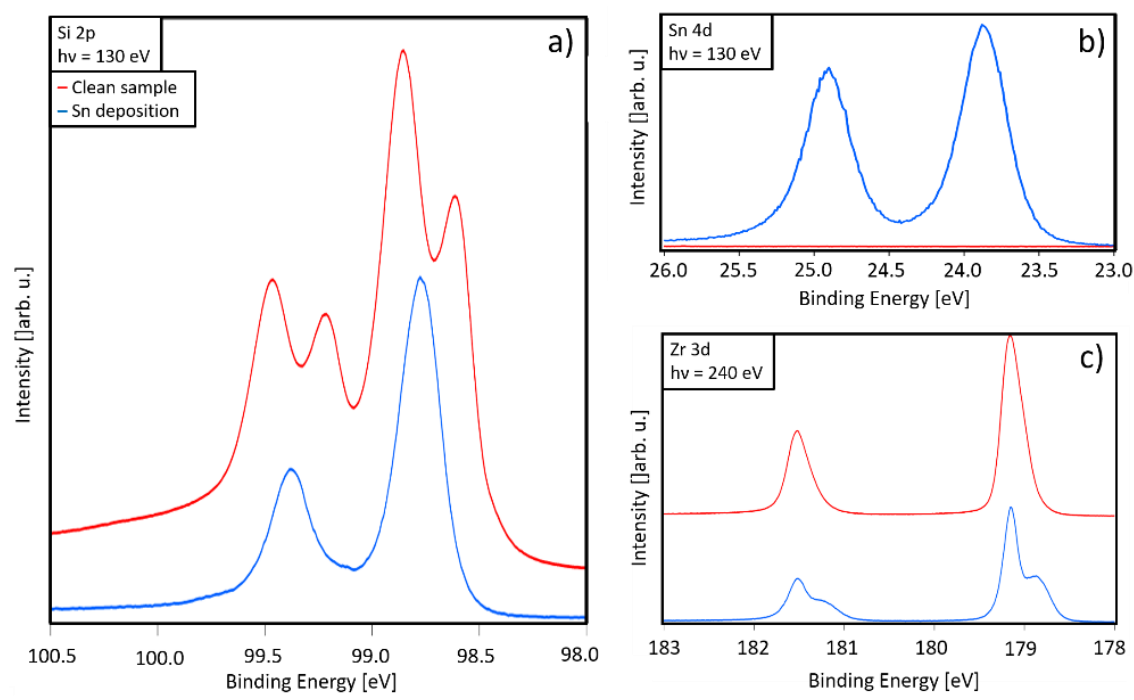

Fig. 3.7 Si $2 p$, Sn $4 d$ and $\mathrm{Zr} 3 d$ core level spectra before and after deposition of Sn and annealing within the temperature range between $400^{\circ} \mathrm{C}$ and $500^{\circ} \mathrm{C}$. The new doublet in the $\mathrm{Zr} 3 d$ spectrum that appears on the low BE side of the main doublet suggests direct contact between $\mathrm{Zr}$ and $\mathrm{Sn}$. The Si $2 p$ core level spectrum changed its shape considerably.

The ARPES spectrum measured after Sn deposition and annealing within the temperature range between $400^{\circ} \mathrm{C}$ and $500^{\circ} \mathrm{C}$ revealed several differences in the band structure compared to that of the pristine sample. Fig. 3.8 compares previously reported calculations of a clean sample band structure of silicene on $\mathrm{ZrB}_{2}$ [33] with our experimental data. While bands $\mathrm{S}_{1}$ and $\mathrm{S}_{4}$ originate from the $d$ orbitals of the outermost $\mathrm{Zr}$ layer (i.e. surface states of $\mathrm{ZrB}_{2}$ ), bands $\mathrm{X}_{2}, \mathrm{X}_{3}$ and $\mathrm{X}_{6}$ have significant $\mathrm{Si} p_{z}$ orbital contributions and hence are associated the silicene. $\mathrm{X}_{5}$ is a hybrid state derived from $\mathrm{Si}$ 
$p$ and $\mathrm{Zr} d$ orbitals. The deposition of Sn heavily influenced Si bands, in particular causing the bands near the $K$ point to disappear. Band $\mathrm{X}_{5}$ shifts to higher binding energy. Similar shifts of this band have been observed for $\mathrm{K}$ deposition on silicene/ $\mathrm{ZrB}_{2}$, attributed to electron donation to $\mathrm{X}_{5}$. [34] The strongly altered $\mathrm{Zr} d$ bands, in particular $\mathrm{S}_{4}$, again suggest direct contact between the deposited $\mathrm{Sn}$ atoms and $\mathrm{Zr}$ atoms. Additionally, new, weakly dispersing bands $\mathrm{Y}_{1}$ and $\mathrm{Y}_{2}$ appear near the $K$ point of the former silicene lattice, which we associate with the new structure. They reside between $0.5-1.5 \mathrm{eV}$ below $E_{F}$, suggesting the structure is semiconducting.

a)

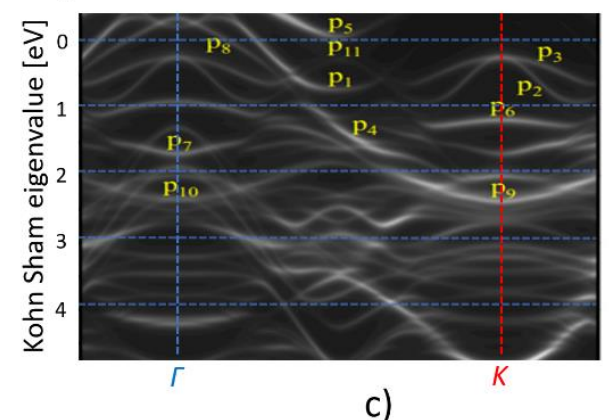

b)

c)
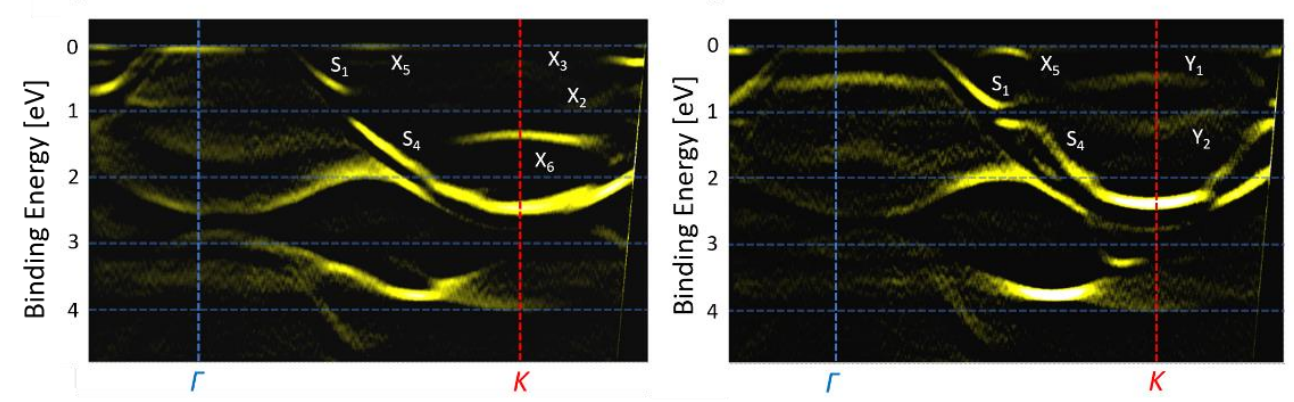

Fig 3.8: a) Calculated electronic band structure of silicene terminated $\mathrm{ZrB}_{2}$ sample, reproduced from [31]. ARPES spectra showing band dispersions in the $\Gamma-K_{S i}$ direction of (b) a clean sample and (c) the sample after Sn deposition followed by annealing within the temperature range of $400^{\circ} \mathrm{C}$ to $500^{\circ} \mathrm{C}$. Band numbers in (b-c) correspond to the numbers in (a). S, $\mathrm{X}$ and $\mathrm{Y}$ bands correspond to Zr-, silicene- and Sn origin, respectively. 


\subsection{4 $500-650^{\circ} \mathrm{C}$ Regime}

Annealing at temperatures higher than $500^{\circ} \mathrm{C}$ causes partial removal of $\mathrm{Sn}$, presumably via surface diffusion or sublimation. In PES spectra, this manifests itself in a gradual and eventually complete suppression of the $\mathrm{Sn} 4 d$ core level peaks, as well as a recovery of the Si $2 p$ core level peaks with a shape characteristic for native silicene. At temperatures above $650^{\circ} \mathrm{C}$ no Sn signal could be detected in PES measurements. The reason for that is related to the properties of the substrate - annealing a silicene/ $/ \mathrm{ZrB}_{2}$ sample at $650^{\circ} \mathrm{C}$ for $15 \mathrm{~min}$ after removal of the silicene layer by ion bombardment has been shown to regenerate the native surface reconstruction [22]. This temperature was concluded to be above the thermal threshold for surface segregation of $\mathrm{Si}$ atoms from the silicon substrate. That coincides with the partial removal of Sn from the surface when annealed close to that temperature. Interestingly, similar temperatures are used to stimulate ordering of $\mathrm{Sn}$ atoms on $\mathrm{Si}(111)$ [35][36]. Hence, in the window between 500 and $650{ }^{\circ} \mathrm{C}$, the temperature seems to play crucial role in what processes take place on the sample.

Annealing in the range between $500-600^{\circ} \mathrm{C}$ lead to another new LEED pattern. As the removal of $\mathrm{Sn}$ was significant during the annealing process, the amount of $\mathrm{Sn}$ left on the surface is unknown. However, it is easily detectable by PES, hence we assume it is still non-negligible. Fig. 3.9e shows the LEED pattern after the annealing process. The original pattern of the pristine sample has partially recovered, which can be seen by recovery of silicene $\sqrt{ } 3$ spot. This shows that more of the Si has been exposed and $\mathrm{Sn}$ was partially removed, possibly due to the aforementioned segregation of Si atoms. In addition to the original pattern a set of new spots appeared. The new spots correspond to a $3 \times 3$ reconstruction with respect to the silicene lattice. It is a remarkable result, when viewed in the context of the well documented $\mathrm{Sn} / \mathrm{Si}(111)$ system. The two outermost $\mathrm{Si}$ layers of the $\mathrm{Si}(111)$ surface plane are structurally very close to silicene [37]. Sn adsorption on that substrate has been investigated for several decades [17][19][35][38][39]. One of the surface reconstructions of that system is the $\sqrt{3}$ $x \sqrt{3} R 30^{\circ}$ reconstruction, which, at low temperature, shows signs of a coexisting 3 x 3 phase [40]. That phase has been reported to be in a Mott-Hubbard insulating state [41][42]. The signature of that state, however, can be only measured in (AR)PES measurements, while STM and LEED do not show any trace of a $3 \times 3$ reconstruction. A few possible origins for the discrepancy have been reported, involving a magnetically-ordered state [43] or magnetic- [44], fast charge- [32] as well as thermal [39] fluctuations. We note that while the system is similar, there are several critical 
differences - silicene is 1 ML thick, it is in a planar - like form (see Fig. 3.1), and the presence of the underlying substrate influences the electronic structure of the system. Nevertheless, we consider the comparison helpful, as it gives important insights into possible interactions between the Sn monolayer and $\mathrm{Si}$ in a system with a honeycomb lattice.

In contradiction to those results, in the case of our experiment the STM images (Fig. $3.9 \mathrm{a}-\mathrm{d}$ ) indeed revealed new structures that can be correlated with the new spots in the LEED pattern. The images provide also an estimation of the coverage. We can therefore state, that the coverage of the sample is below $1 / 3$ of ML. Such statement is important due to the comparison between our system and $\mathrm{Sn} / \mathrm{Si}(111)$ system, where $1 / 3$ of ML is a threshold above which an $2 \sqrt{ } 3 \times 2 \sqrt{ } 3 \mathrm{R} 30^{\circ}$ phase appears. The apparent low coverage of our sample makes the comparison and the conclusions drawn from it more straight forward. The observed structures mostly originate from step edges, or form (less frequently) as isolated islands (see Fig. 3.9a). FFT performed on the STM images shows clearly that the islands are comprised of the new reconstruction, responsible for the $3 x$ 3 spots in the LEED images. The structure exhibits repeating units of 3 atoms in a row, which are arranged such that they are forming a $3 \times 3$ lattice with the lattice constant $\lambda=10.95 \AA$. It is likely that the unit cell contains more atoms that are not resolved by the STM method. Fig. 3.10 shows that the STM images of the new reconstruction are heavily bias dependent. While the clearly atomically resolved structures shown in Fig. 9 were taken at $77 \mathrm{~K}$, the LEED pattern was measured at RT and it still shows the $3 \times 3$ spots. Similarly, RT - STM measurements show structures with distorted $3 \times 3$ periodicity. This shows that, while the temperature plays a role, the structure is stable within a wide range of temperatures, in particular at room temperature. 

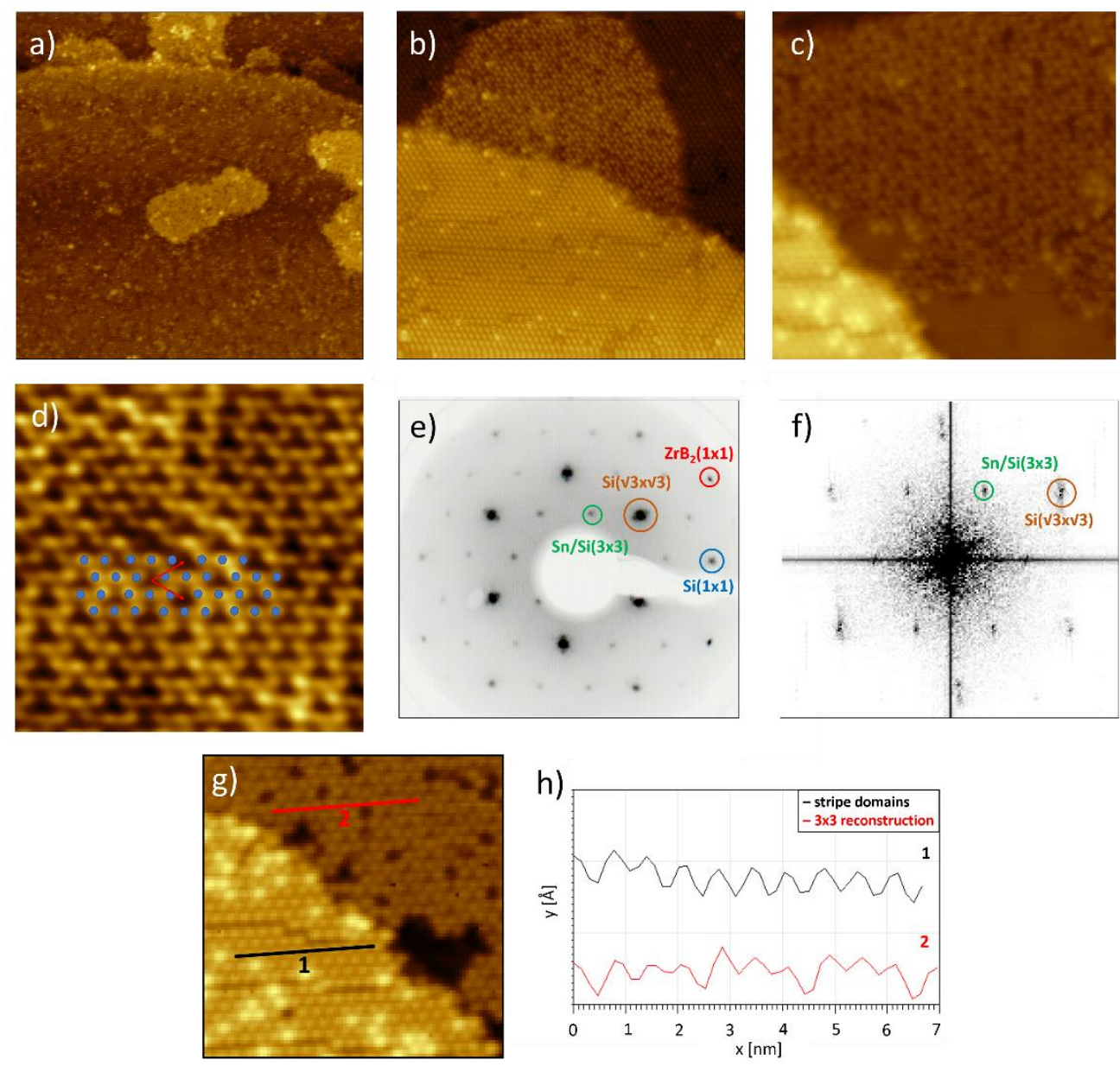

Fig. 3.9 a) STM image of Sn structures growing from a step edge as well as in the form of islands. Note that the surrounding structure is blurred due to the imperfect tip quality $\left(90 \times 90 \mathrm{~nm}^{2}\right.$, $\mathrm{V}_{\text {bias }}=+0.7 \mathrm{~V}, \mathrm{~T}=300 \mathrm{~K}$ ). Despite that, it is possible to see the stripe domain silicene structure, which has partially recovered. b) STM image $\left(40 \times 40 \mathrm{~nm}^{2}, \mathrm{~V}_{\text {Bias }}=+1.0 \mathrm{~V}, \mathrm{~T}=300 \mathrm{~K}\right)$ of the $3 \times 3$ structure grown from the step edge. A clear recovery of the stripe domains is seen, as well as $3 \times 3$ periodicity, which is easily seen in the c) FFT-filtered STM image $\left(10 \times 10 \mathrm{~nm}^{2}, \mathrm{~V}_{\text {Bias }}=\right.$ $+1.0 \mathrm{~V}, \mathrm{~T}=77 \mathrm{~K}$ ). d) A ball model of the atomic lattice is displayed on top of the FFT-filtered STM image of the ordered Sn-derived structure, with red arrows marking the base vectors of the unit cell. The lattice constant of the structure is estimated to be $10.95 \AA$. e) LEED $\left(E_{B}=40\right.$ eV) images of the sample after $\mathrm{Sn}$ deposition followed by annealing at $550{ }^{\circ} \mathrm{C}$, showing a new pattern. Note that while the $\mathrm{Si} \sqrt{ } 3 \times \sqrt{ } 3$ spots are less distinct, they keep their original arrangement of fractional spots. This suggests that the coverage of $\mathrm{Sn}$ on the surface is very low, as most of the 
surface preserves the stripe domain structure, which is the origin of the fractional spots. f) The 2D Fast Fourier Transform of c) matches perfectly with the LEED pattern of e). g) STM $\left(2 \times 2 \mathrm{~nm}^{2}, \mathrm{~V}_{\text {bias }}=-2.0 \mathrm{~V}\right)$ image of the $3 \times 3$ reconstruction next to the exposed native silicene. $\left.\mathrm{h}\right)$ line profiles indicated by lines 1 (black) and 2 (red) in the STM image. Note the 3-atom periodicity in the line profile (red) of the new structure.
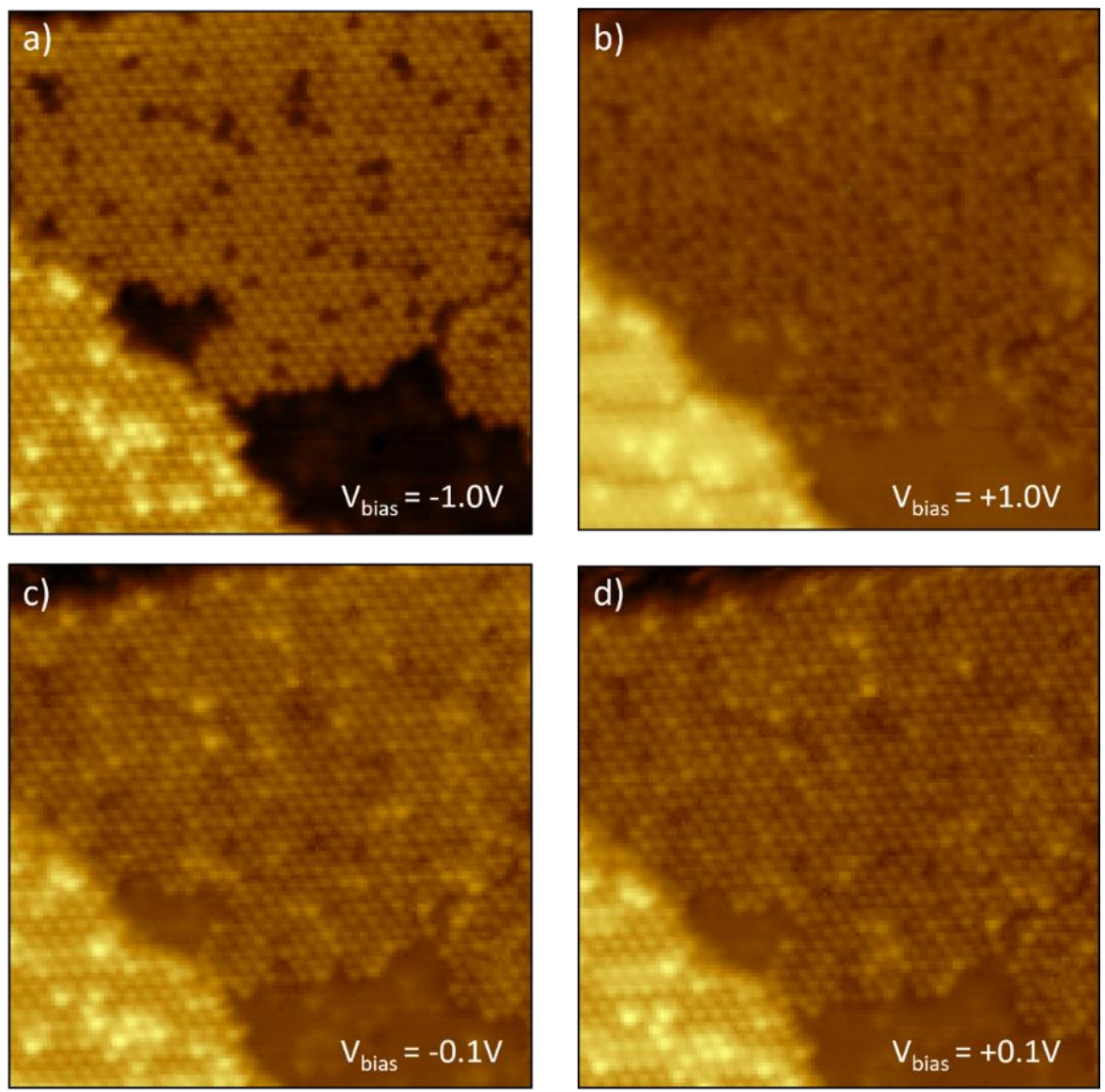

Fig. 3.10: (a,b,d,e) STM images (each $20 \times 20 \mathrm{~nm}^{2}$, T = $77 \mathrm{~K}$ ) showing the bias dependence of the new structure. The lower left corner of the images corresponds to silicene with stripe domains and many adatoms of Si or Sn. On the right, the new structure can be observed. Interestingly, the $3 \times 3$ periodicity is clearly visible at high positive bias voltage, while at low or negative bias voltages this periodicity is hardly visible at all. 
a)

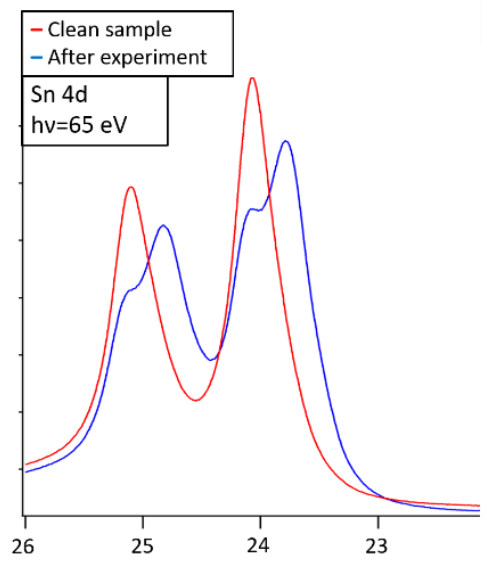

C)

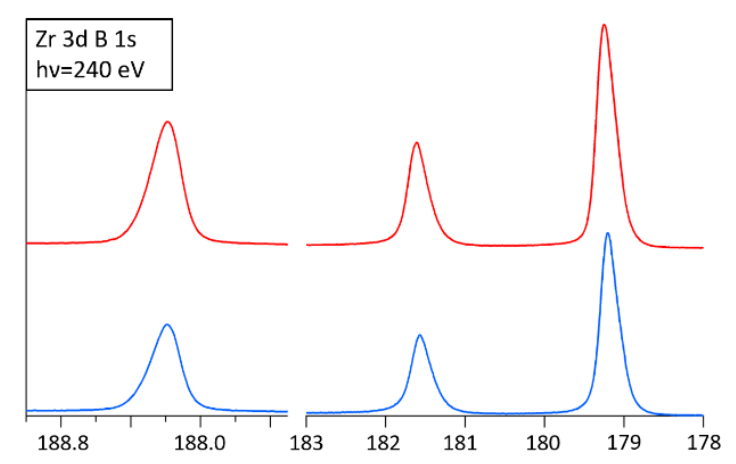

b)

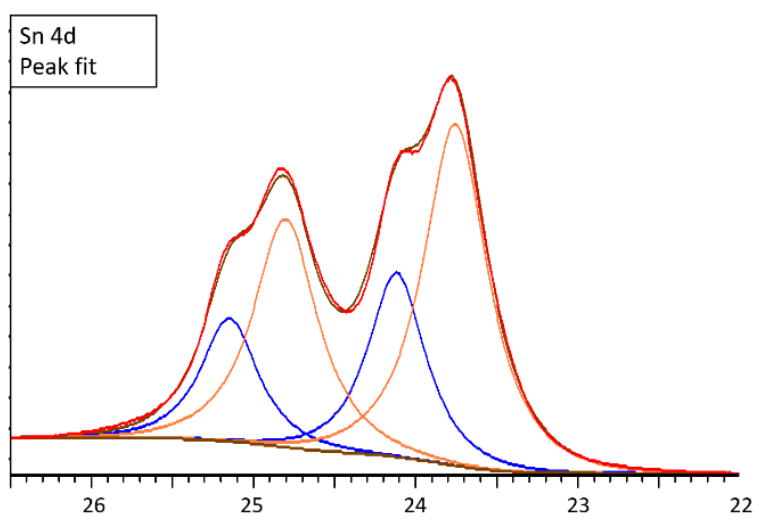

d)

Fig. 3.11: XPS of Sn $4 d(\mathrm{a}-\mathrm{b}), \mathrm{Zr} 3 d$ and B $1 s$ (c) and Si $2 p$ (d) core levels of a clean sample (red) and after Sn deposition and annealing between $500^{\circ} \mathrm{C}$ and $650^{\circ} \mathrm{C}$. The Sn core level shows an extra peak at the lower binding energy, suggesting a new chemical species of $\mathrm{Sn}$. The $\mathrm{Si}, \mathrm{Zr}$ and B peaks look very similar to the original ones, which suggests very little interaction with $\mathrm{Sn}$ atoms, probably due to the low Sn coverage caused by partial removal of Sn atoms during annealing.

PES measurements were performed to get an insight into the chemical composition of the new structure (Fig. 11). The formation of the $3 \times 3$ structure coincides with the emergence of an additional component in the $\mathrm{Sn} 4 d$ core level spectrum. This 
component resembles the additional component found in the case of the $\sqrt{ } 3 \times \sqrt{ } 3 \mathrm{R} 30^{\circ}$ reconstruction of the $\mathrm{Sn} / \mathrm{Si}(111)$ system. In that system, the higher binding energy component was associated with metallic $\mathrm{Sn}$ in $\sqrt{ } 3 \times \sqrt{ } 3 \mathrm{R} 30^{\circ}$ phase, while the lower binding energy component was identified as the $3 \times 3$ phase, possibly semiconducting [17] [39] or Mott-insulating [42]. We note that in the case of that system, the component was measured at low temperature (below $70 \mathrm{~K}$ ), while our measurements were done at room temperature. The new component is shifted with respect to the original position by $350 \mathrm{meV}$ in the direction of the lower binding energy. The new component implies a new chemical species of $\mathrm{Sn}$, which is a good indication that this new structure involves Sn atoms.

In the case of the silicene and substrate peaks - one can observe their recovery to the original shapes. This is a result of partial removal of $\mathrm{Sn}$ atoms from the surface and recovery of native silicene. It is consistent with STM observations, as well as with the recovery of the silicene spots in the LEED pattern (to see the evolution of the LEED pattern, compare Fig 3.9e with Fig. 3.5c and Fig. 3.1d).

ARPES measurements were performed on the surface, however no new bands that could be correlated with the $3 \times 3$ structure were detected, when compared with the spectrum of a clean sample. A possible explanation is a misalignment of LEED and ARPES measurements resulting in measurements in different locations. Another plausible reason might be the partial $\mathrm{Sn}$ coverage of the surface of the sample due to the removal of $\mathrm{Sn}$ as a result of annealing. Such partial coverage could be too small to produce discernible bands in the ARPES measurements.

Since no new bands could be identified in ARPES measurements, we used more local measurements that would give insight in the electronic properties of the new structure, especially regarding its conducting properties. The STS measurement shown in Fig. 3.12 shows a wide featureless gap of about $1.2 \mathrm{eV}$. While the gap is clearly observed, there is a residual conductance inside it. It most probably is a contribution from the metallic substrate, since the position of the bump inside the gap matches the position of the substrate signal in the STS measurement of the clean sample (see Fig. 3.1e). This STS curve is also significantly different from the previously reported STS measurement of the $\mathrm{Sn} / \mathrm{Si}(111)$ system [31]. In addition, the difference in temperature used in our 
work and that report, as well as the clear contribution from the substrate in our experiment, make the comparison difficult.
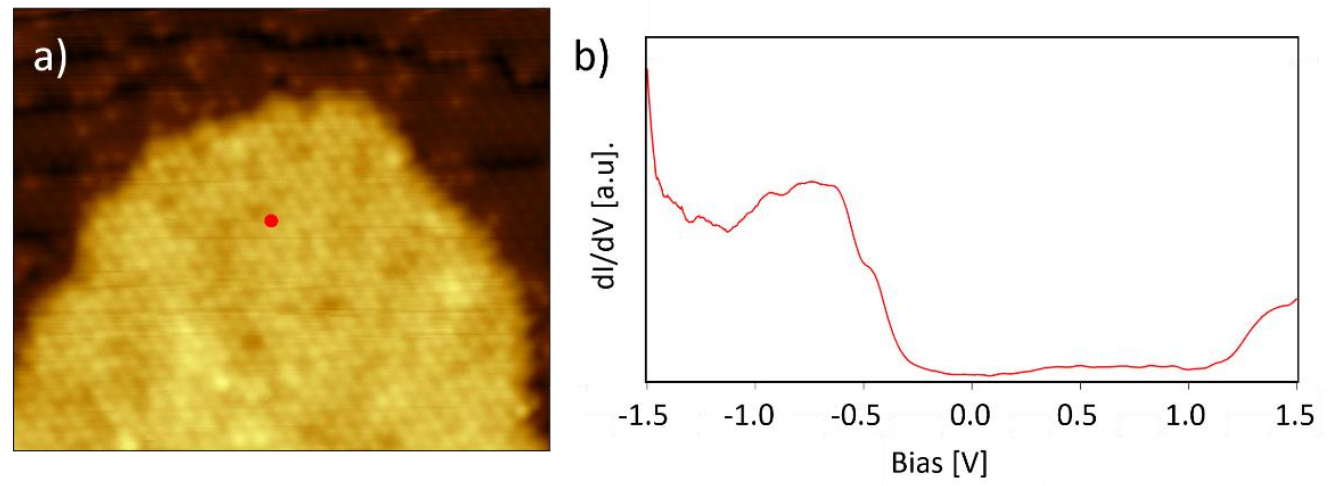

Fig. 3.12: a) STM $\left(20 \times 20 \mathrm{~nm}^{2}, \mathrm{~V}_{\text {bias }}=+1.5 \mathrm{~V}\right)$ image with STS measurement location indicated by the red dot. b) STS spectrum reveals a wide featureless gap between $-300 \mathrm{meV}$ to $1200 \mathrm{meV}$. The finite signal inside this gap is attributed to tunneling involving $\mathrm{ZrB}_{2}$ - derived bands, marked by the bump at $750 \mathrm{meV}$.

\subsection{Conclusions}

Table 3.I Summary of LEED patterns and structures measured by STM after deposition of approximately $1 \mathrm{ML}$ of Sn the sample and consecutive annealing at temperatures indicated in the table.

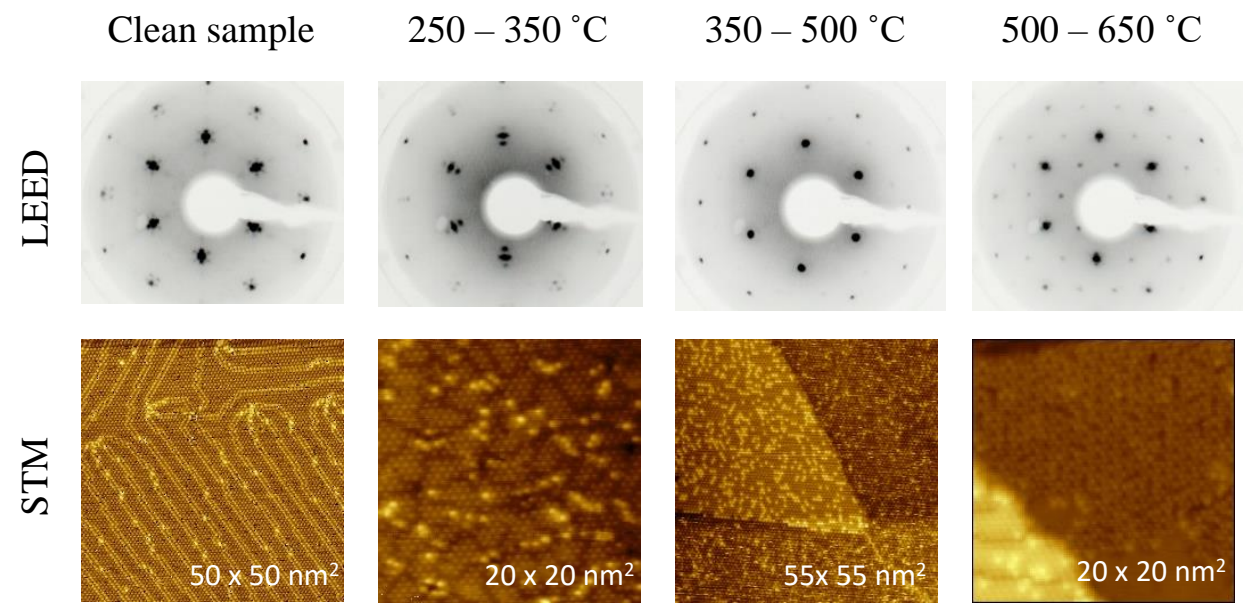


We report on an investigation of new 2D structures formed by two group IV elements, $\mathrm{Si}$ and $\mathrm{Sn}$, using silicene terminated $\mathrm{ZrB}_{2}$ as a starting point and $\mathrm{Sn}$ deposition followed by annealing to form the new structures. Both $\mathrm{Si}$ and $\mathrm{Sn}$ are known to form epitaxial monolayers with buckled honeycomb lattices, which may exhibit exotic electronic properties. In the temperature range between 250 and $350^{\circ} \mathrm{C}$, we found that $\mathrm{Sn}$ atoms replace $\mathrm{Si}$ atoms that were initially in direct contact with $\mathrm{ZrB}_{2}$, freeing them to form structures that are very similar to the ones obtained after additional Si deposition. In particular, a metallic Si-based layer with a typical quasi triangular pattern is formed. This layer, which presumably resides on top of silicene forming a Si bilayer, is essentially identical to that reported in a previously published paper that describes $\mathrm{Si}$ deposition on the same surface. Interestingly, we found another way to form such a layer exploiting the chemical interactions between different elements. Using Sn to obtain an excess of $\mathrm{Si}$ atoms on the surface involves removal of these $\mathrm{Si}$ atoms from their original location. Hence our method shows that Sn, in principle, can be used to etch and pattern the silicene surface with the formation of a Si bilayer. Such a procedure may be used to form lateral 2D heterostructures, for example metallic contacts to a silicene layer in future silicene - based devices.

Annealing to temperatures between $400-500^{\circ} \mathrm{C}$ results in disintegration of the $\mathrm{Si}$ bilayer and the formation of alloy - like structures, with $\mathrm{Sn}$ atoms incorporated into the $\mathrm{Si}$ lattice in a seemingly random manner. This is the first example of forming a $\mathrm{Sn}-\mathrm{Si}$ alloy in 2D form. It opens the door for future tuning of the electronic properties, e.g. by adjusting the ratio of the elements in the alloy or by patterning the alloy [45]. The alloy shows also strong bias dependence in STM images. The alloy islands are surrounded by a "blurry" structure with unknown exact composition. The inability to obtain sharp STM images, while ARPES and LEED data show ordered phases and clear new bands, resembles a well-known phenomenon for the $\mathrm{Sn} / \mathrm{Si}(111)$ system, which was recently explained by the high mobility of Sn atoms and the speed with which different techniques probe the surface (STM is slow compared to PES and LEED).

Finally, we found that annealing above $500^{\circ} \mathrm{C}$ creates a new structure, with a lattice periodicity corresponding to a $3 \times 3$ reconstruction with respect to the lattice of silicene. This structure appears in the form of small islands that predominantly grow from step edges. STS measurements of these islands show a $1.2 \mathrm{eV}$ wide "gap" with sharp edges, which is large in comparison to any experimental or theoretical report of a hexagonal monolayer of group IV elements. To our knowledge this is the first ever report of an 
experimentally realized layered heterostructure of two group IV elements beyond graphene in the 2D regime. As 2D materials in the group IV (silicene, germanene, stanene) are predicted to exhibit exotic properties, our findings shine light on the perspective of combining them into one system. Our pioneer investigation enriches the spectrum of $2 \mathrm{D}$ materials and shows a direction for exploring a new branch of heterostructures.

\section{References}

[1] K. S. Novoselov, "Electric Field Effect in Atomically Thin Carbon Films," Science, vol. 306, no. 5696, pp. 666-669, Oct. 2004, doi: 10.1126/science.1102896.

[2] P. Vogt et al., "Silicene: Compelling Experimental Evidence for Graphenelike Two-Dimensional Silicon," Phys. Rev. Lett., vol. 108, no. 15, p. 155501, Apr. 2012, doi: 10.1103/PhysRevLett.108.155501.

[3] A. Fleurence, R. Friedlein, T. Ozaki, H. Kawai, Y. Wang, and Y. YamadaTakamura, "Experimental Evidence for Epitaxial Silicene on Diboride Thin Films," Phys. Rev. Lett., vol. 108, no. 24, p. 245501, Jun. 2012, doi: 10.1103/PhysRevLett.108.245501.

[4] L. Meng et al., "Buckled Silicene Formation on $\operatorname{Ir}(111)$," Nano Lett., vol. 13, no. 2, pp. 685-690, Feb. 2013, doi: 10.1021/nl304347w.

[5] M. E. Dávila, L. Xian, S. Cahangirov, A. Rubio, and G. Le Lay, "Germanene: a novel two-dimensional germanium allotrope akin to graphene and silicene," New J. Phys., vol. 16, no. 9, p. 095002, Sep. 2014, doi: 10.1088/1367-2630/16/9/095002.

[6] L. Li et al., "Buckled Germanene Formation on Pt(111)," Adv. Mater., vol. 26, no. 28, pp. 4820-4824, Jul. 2014, doi: 10.1002/adma.201400909.

[7] L. Zhang et al., "Structural and Electronic Properties of Germanene on MoS 2," Phys. Rev. Lett., vol. 116, no. 25, p. 256804, Jun. 2016, doi: 10.1103/PhysRevLett.116.256804.

[8] F. Zhu et al., "Epitaxial growth of two-dimensional stanene," Nat. Mater., vol. 14, no. 10, pp. 1020-1025, Oct. 2015, doi: 10.1038/nmat4384. 
[9] S. Saxena, R. P. Chaudhary, and S. Shukla, "Stanene: Atomically Thick Freestanding Layer of 2D Hexagonal Tin," Sci. Rep., vol. 6, no. 1, p. 31073, Nov. 2016, doi: 10.1038/srep31073.

[10] J. Gou et al., "Strain-induced band engineering in monolayer stanene on Sb(111)," Phys. Rev. Mater., vol. 1, no. 5, p. 054004, Oct. 2017, doi: 10.1103/PhysRevMaterials.1.054004.

[11] M. Ezawa, "Monolayer Topological Insulators: Silicene, Germanene, and Stanene," J. Phys. Soc. Jpn., vol. 84, no. 12, p. 121003, Dec. 2015, doi: 10.7566/JPSJ.84.121003.

[12] S. Zaima, O. Nakatsuka, N. Taoka, M. Kurosawa, W. Takeuchi, and M. Sakashita, "Growth and applications of GeSn-related group-IV semiconductor materials," Sci. Technol. Adv. Mater., vol. 16, no. 4, p. 043502, Jul. 2015, doi: 10.1088/1468-6996/16/4/043502.

[13] S. Wirths, D. Buca, and S. Mantl, "Si-Ge-Sn alloys: From growth to applications," Prog. Cryst. Growth Charact. Mater., vol. 62, no. 1, pp. 1-39, Mar. 2016, doi: 10.1016/j.pcrysgrow.2015.11.001.

[14] V. O. Ozcelik, D. Kecik, E. Durgun, and S. Ciraci, "Adsorption of Group IV Elements on Graphene, Silicene, Germanene, and Stanene: Dumbbell Formation," J. Phys. Chem. C, vol. 119, no. 1, pp. 845-853, Jan. 2015, doi: 10.1021/jp5106554.

[15] P. Moontragoon et al., "Electronic properties calculation of Ge1-x-ySixSny ternary alloy and nanostructure," J. Non-Cryst. Solids, vol. 358, no. 17, pp. 2096-2098, Sep. 2012, doi: 10.1016/j.jnoncrysol.2012.01.025.

[16] R. Zhachuk, S. Teys, and J. Coutinho, "Strain-induced structure transformations on $\mathrm{Si}(111)$ and $\mathrm{Ge}(111)$ surfaces: a combined density-functional and scannning tunnneling microscopy report," J. Chem. Phys., vol. 138, no. 22, p. 224702, Jun. 2013, doi: $10.1063 / 1.4808356$.

[17] M. Göthelid, M. Björkqvist, T. M. Grehk, G. Le Lay, and U. O. Karlsson, "Metal-semiconductor fluctuation in the $\mathrm{Sn}$ adatoms in the $\mathrm{Si}(111)-\mathrm{Sn}$ and $\mathrm{Ge}(111)$ Sn $(\sqrt{ } 3 \times \sqrt{ } 3) \mathrm{R} 30^{\circ}$ reconstructions,” Phys. Rev. B, vol. 52, no. 20, pp. R14352 R14355, Nov. 1995, doi: 10.1103/PhysRevB.52.R14352. 
[18] Y. Yamada-Takamura, F. Bussolotti, A. Fleurence, S. Bera, and R. Friedlein, "Surface electronic structure of ZrB2 buffer layers for GaN growth on Si wafers," Appl. Phys. Lett., vol. 97, no. 7, p. 073109, Aug. 2010, doi: 10.1063/1.3481414.

[19] A. Taleb-Ibrahimi, C. A. Sebenne, F. Proix, and P. Maigne, "Adsorption of Sn on cleaved Si(111) surfaces," Surf. Sci., vol. 163, no. 2, pp. 478-488, Nov. 1985, doi: 10.1016/0039-6028(85)91073-8.

[20] B. van den Broek, M. Houssa, E. Scalise, G. Pourtois, V. V. Afanas'ev, and A. Stesmans, "Two-dimensional hexagonal tin: ab initio geometry, stability, electronic structure and functionalization," 2D Mater., vol. 1, no. 2, p. 021004, Aug. 2014, doi: 10.1088/2053-1583/1/2/021004.

[21] R. Friedlein and Y. Yamada-Takamura, "Electronic properties of epitaxial silicene on diboride thin films," J. Phys. Condens. Matter, vol. 27, no. 20, p. 203201, May 2015, doi: 10.1088/0953-8984/27/20/203201.

[22] A. Fleurence and Y. Yamada-Takamura, "Insights into the spontaneous formation of silicene sheet on diboride thin films," Appl. Phys. Lett., vol. 110, no. 4, p. 041601, Jan. 2017, doi: 10.1063/1.4974467.

[23] C.-C. Lee et al., "Single-particle excitation of core states in epitaxial silicene," Phys. Rev. B, vol. 95, no. 11, p. 115437, Mar. 2017, doi: 10.1103/PhysRevB.95.115437.

[24] A. Fleurence et al., "Single-domain epitaxial silicene on diboride thin films," Appl. Phys. Lett., vol. 108, no. 15, p. 151902, Apr. 2016, doi: 10.1063/1.4945370.

[25] C.-C. Lee, A. Fleurence, R. Friedlein, Y. Yamada-Takamura, and T. Ozaki, "Avoiding critical-point phonon instabilities in two-dimensional materials: The origin of the stripe formation in epitaxial silicene," Phys. Rev. B, vol. 90, no. 24, p. 241402, 2014, doi: 10.1103/PhysRevB.90.241402.

[26] M. Nogami, A. Fleurence, Y. Yamada-Takamura, and M. Tomitori, "Nanomechanical Properties of Epitaxial Silicene Revealed by Noncontact Atomic Force Microscopy," Adv. Mater. Interfaces, vol. 6, no. 2, p. 1801278, Jan. 2019, doi: 10.1002/admi.201801278. 
[27] R. Friedlein et al., "Core level excitations-A fingerprint of structural and electronic properties of epitaxial silicene," J. Chem. Phys., vol. 140, no. 18, p. 184704, May 2014, doi: 10.1063/1.4875075.

[28] T. G. Gill et al., "Metallic atomically-thin layered silicon epitaxially grown on silicene/ZrB \textlesssubltextgreater2\textless/subltextgreater," 2D Mater., vol. 4, no. 2, p. 021015 , Feb. 2017, doi: 10.1088/2053-1583/aa5a80.

[29] A. Fleurence et al., "Bandgap engineering in an epitaxial two-dimensional honeycomb Si6-xGex alloy," p. 13.

[30] A. Charrier et al., "Contrasted electronic properties of Sn-adatom-based ( $3 \times$ 3 ) R $30^{\circ}$ reconstructions on Si(111)," Phys. Rev. B, vol. 64, no. 11, p. 115407, Aug. 2001, doi: 10.1103/PhysRevB.64.115407.

[31] B. Ressel, C. Di Teodoro, G. Profeta, L. Ottaviano, V. Cháb, and K. C. Prince, "Scanning tunneling spectroscopy investigation of the $(\sqrt{ } 3 \times \sqrt{ } 3) \mathrm{R} 30^{\circ} \quad \mathrm{Sn} / \mathrm{Si}(111)$ $\alpha$ and $\gamma$ surfaces," Surf. Sci., vol. 562, no. 1-3, pp. 128 - 136, Aug. 2004, doi: 10.1016/j.susc.2004.05.121.

[32] P. Hansmann, T. Ayral, A. Tejeda, and S. Biermann, "Uncertainty principle for experimental measurements: Fast versus slow probes," Sci. Rep., vol. 6, no. 1, p. 19728, Apr. 2016, doi: 10.1038/srep19728.

[33] C.-C. Lee, A. Fleurence, Y. Yamada-Takamura, T. Ozaki, and R. Friedlein, "Band structure of silicene on the zirconium diboride (0001) thin film surface convergence of experiment and calculations in the one-Si-atom Brillouin zone," Phys. Rev. B, vol. 90, no. 7, p. 075422, 2014, doi: 10.1103/PhysRevB.90.075422.

[34] R. Friedlein, A. Fleurence, J. T. Sadowski, and Y. Yamada-Takamura, "Tuning of silicene-substrate interactions with potassium adsorption," Appl. Phys. Lett., vol. 102, no. 22, p. 221603, Jun. 2013, doi: 10.1063/1.4808214.

[35] T. Ichikawa, "Structural study of ultrathin Sn layers deposited onto Ge(111) and Si(111) surfaces by RHEED,” Surf. Sci., vol. 140, no. 1, pp. 37-63, 1984. 
[36] C. Törnevik et al., "Adsorption of $\mathrm{Sn}$ on $\mathrm{Si}(111) 7 \times 7$ : reconstructions in the monolayer regime," Surf. Sci., vol. 314, no. 2, pp. 179-187, Jul. 1994, doi: 10.1016/0039-6028(94)90005-1.

[37] G. G. Guzmán-Verri and L. C. Lew Yan Voon, "Electronic structure of siliconbased nanostructures," Phys. Rev. B, vol. 76, no. 7, p. 075131, Aug. 2007, doi: 10.1103/PhysRevB.76.075131.

[38] C. Törnevik, M. Hammar, N. G. Nilsson, and S. A. Flodström, “Epitaxial growth of $\mathrm{Sn}$ on $\mathrm{Si}(111)$ : A direct atomic-structure determination of the $(2 \sqrt{ } 3 \times 2 \sqrt{ }$ 3 ) R $30^{\circ}$ reconstructed surface,” Phys. Rev. B, vol. 44, no. 23, pp. 13144 - 13147, Dec. 1991, doi: 10.1103/PhysRevB.44.13144.

[39] R. I. G. Uhrberg, H. M. Zhang, T. Balasubramanian, S. T. Jemander, N. Lin, and G. V. Hansson, "Electronic structure of S n / S i ( 111 ) $3 \times 3$ : Indications of a lowtemperature phase," Phys. Rev. B, vol. 62, no. 12, pp. 8082-8086, Sep. 2000, doi: 10.1103/PhysRevB.62.8082.

[40] L. Ottaviano, "STM investigation of the a-Sn/Si(111) phase at $120 \mathrm{~K}$," Surf. Sci., p. 6, 2000.

[41] G. Profeta, L. Ottaviano, S. Santucci, and A. Continenza, "Two-dimensional alloying on Si(111) surface: An ab initio study," Phys. Rev. B, vol. 66, no. 8, p. 081303 , Aug. 2002, doi: 10.1103/PhysRevB.66.081303.

[42] S. Modesti et al., "Insulating Ground State of Sn / Si ( 111 ) - ( $3 \times 3$ ) R 30 ०," Phys. Rev. Lett., vol. 98, no. 12, p. 126401, Mar. 2007, doi: 10.1103/PhysRevLett.98.126401.

[43] G. Li et al., "Magnetic order in a frustrated two-dimensional atom lattice at a semiconductor surface," Nat. Commun., vol. 4, no. 1, p. 1620, Jun. 2013, doi: $10.1038 /$ ncomms 2617 .

[44] S. Glass et al., "Triangular Spin-Orbit-Coupled Lattice with Strong Coulomb Correlations: Sn Atoms on a SiC(0001) Substrate,” Phys. Rev. Lett., vol. 114, no. 24, p. 247602, Jun. 2015, doi: 10.1103/PhysRevLett.114.247602. 
[45] K. Nakada, M. Fujita, G. Dresselhaus, and M. S. Dresselhaus, "Edge state in graphene ribbons: Nanometer size effect and edge shape dependence," Phys. Rev. B, vol. 54, no. 24, pp. 17954-17961, Dec. 1996, doi: 10.1103/PhysRevB.54.17954. 




\section{CHAPTER 4}

\section{Epitaxial Sn on $\mathrm{MoS}_{2}$}

\subsection{Introduction}

The Sn analogue of graphene - stanene is one of the most promising materials among new 2D topological materials. As described in chapter 3, graphene-like Sn layers preserve most of the exotic properties observed in graphene, while it also has been predicted to exhibit the quantum spin Hall effect (QSHE) at room temperature due to its much larger spin orbit coupling (SOC). Epitaxial growth is the most commonly embraced method to obtain a Sn honeycomb monolayer. A few reports [1][2][3] have suggested $\mathrm{MoS}_{2}$ as a suitable substrate, able to support stanene without destructive influence on its electronic properties. $\mathrm{MoS}_{2}$ is a layered, graphite-like semiconductor with an indirect band gap of $1.2 \mathrm{eV}$ in bulk form, and a direct band gap of $1.8 \mathrm{eV}$ in the case of monolayers. Its lattice constant of $3.166 \AA$ gives a favorable lattice mismatch of about $1.45 \%$ for a $3 \times 3\left(\mathrm{MoS}_{2}\right)-2 \times 2$ (stanene) superstructure [3]. The hybridization between $\mathrm{MoS}_{2}$ bands and stanene bands are expected to be weak, as the interlayer bonds in $\mathrm{MoS}_{2}$ are dominated by van der Waals interactions, while the surface is chemically inert. Additionally, other reports suggest that $\mathrm{MoS}_{2}$ is able to support germanene [4], while Si has been shown to intercalate between its layers [5]. In this chapter, we report on our investigation on the feasibility of $\mathrm{MoS}_{2}$ to support stanene.

\subsection{Methods}

The $\mathrm{MoS}_{2}$ sample used in this work was a commercial synthetic $\mathrm{MoS}_{2}$ bulk crystal provided by $2 \mathrm{D}$ Semiconductors. Sample preparation consisted of cleaning the surface 
by mechanical exfoliation and subsequent annealing at a temperature below $200^{\circ} \mathrm{C}$ for $2-3 \mathrm{~h}$ in ultrahigh vacuum (UHV), using a resistive heating setup.

The experiment was performed in our home lab in a UHV system consisting of a load lock, an STM chamber with RHK VT-STM setup, and a separate preparation chamber in which the deposition and annealing treatment was done. The base pressure in the system is lower than $5.0 \times 10^{-10}$ mbar. We deposited Sn from a $99 \%$ pure Sn sheet using a commercial effusion cell. The pressure during sample preparation was lower than 1.5 $\times 10^{-8} \mathrm{mbar}$ and lower than $5.0 \times 10^{-9} \mathrm{mbar}$ during the measurement. Annealing of the sample is done by resistive heating of a tungsten filament close to the backside of the sample. After preparation the sample is transferred in-situ to the STM system. STM measurements were done at room temperature (RT) with a commercial Pt/Ir tip.

\subsection{Results and discussion}

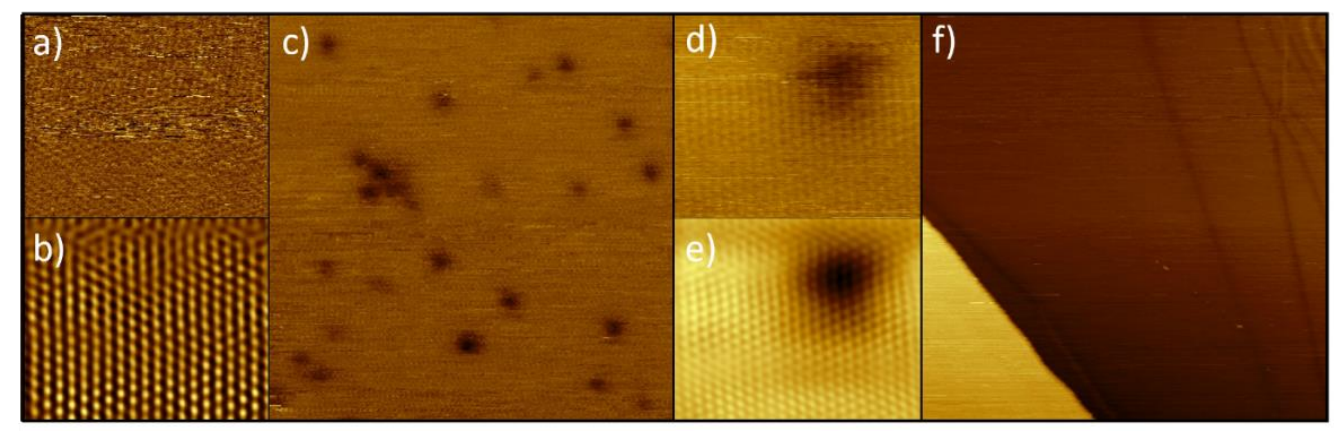

Fig 4.1. Atomically resolved raw (a) and FFT-filtered (b) STM images of the clean $\mathrm{MoS}_{2}$ surface, showing a hexagonal pattern. Larger scale images show native surface feature - defects (c-e) and wrinkles(f). Electronic defects are shown in atomic resolution (d). The FFT- filtered image of (e) shows no s-vacancies inside the defect. (a) $10 \times 9 \mathrm{~nm}^{2}, \mathrm{~V}_{\text {bias }}=1.0 \mathrm{~V}, \mathrm{I}_{\text {stp }}=0.4 \mathrm{nA}$ (c) $40 \times 36$ $\mathrm{nm}^{2}, \mathrm{~V}_{\text {bias }}=1.2 \mathrm{~V}, \mathrm{I}_{\text {stp }}=0.1 \mathrm{nA},(\mathrm{d}) 6 \times 5 \mathrm{~nm}^{2}, \mathrm{~V}_{\text {bias }}=1.0 \mathrm{~V}, \mathrm{I}_{\text {stp }}=0.4 \mathrm{nA}$ (f) $300 \times 270 \mathrm{~nm}^{2}, \mathrm{~V}_{\text {bias }}$ $=1.2 \mathrm{~V}, \mathrm{I}_{\mathrm{stp}}=0.1 \mathrm{nA}$.

Our experiment was aimed at forming a monolayer honeycomb lattice of $\mathrm{Sn}$ - stanene - on top of $\mathrm{MoS}_{2}$. The bulk $\mathrm{MoS}_{2}$ sample was cleaned by exfoliation of the top layers to expose a fresh surface and subsequently introduced to the UHV system. Typical STM images of the so-prepared sample are shown in Fig 4.1. A clean $\mathrm{MoS}_{2}$ surface has hexagonal symmetry with a lattice constant of about $3.2 \AA$. We also could observe 
wrinkles and terraces. The terraces are most probably the effect of the exfoliation procedure. Wrinkles, on the other hand, have their origin in interlayer strain as well as breaks in deeper layers. The surface exhibits also a number of defects. Such defects can be classified as metal - like or structural, based on their appearance in STM measurements [6][7]. The structural defects that STM can directly detect are vacancies in the outermost $\mathrm{S}$ layer - S-vacancies. The metal - like defects are the ones that do not show any rearrangement of the outermost atomic layer. They have been attributed to sub-surface Mo defects, hence the name: metal - like [7]. They are imaged by STM in the form of a disturbance of the electronic structure that extends for a certain distance from the (invisible in STM) physical vacancy. In the latter case the defects will appear on a STM image as regions with darker contrast (see Fig 4.1d-e).

The conditions for formation of germanene on $\mathrm{MoS}_{2}$ [4] were taken as a starting point for our experiment. In that experiment, Ge was deposited on the surface of $\mathrm{MoS}_{2}$ sample at a very low rate of $<0.1 \mathrm{ML} / \mathrm{h}$. In all experiments shown below, the Sn was deposited for $15 \mathrm{~min}$ at a similar rate of $<0.1 \mathrm{ML} / \mathrm{h}$. In the initial experiment, the source was located close to the sample (the source - sample distance was about $5 \mathrm{~cm}$ ), which inadvertently resulted in deposition at an elevated temperature due to radiative heating of the sample by the hot Sn source. However, the geometry of the experiment does not allow for measurements of the temperature.

After the deposition, the surface was examined by means of STM (Fig. 4.2). The deposited Sn formed disordered structures without identifiable crystal facets with droplet - like characteristics. The droplets mostly accumulated along the step edges of the terraces. Possible nucleation sites for these droplets may be surface defects. Note that the clusters are much bigger than the typical size of the defects and should cover them when placed on top, hence the latter are not visible in the STM measurement. Further insight into the nucleation was given by the observation that the STM tip can displace the islands during the measurement. That allowed to scan the surface under the droplet. Fig. 4.2f-g show the displacement of a droplet and the consecutive scan. It is clear that a droplet is pushed by the tip in Fig. $4.2 \mathrm{f}$ and is imaged several times as the apparent string of droplets. In Fig. 4.2g the same area shows only a single droplet in the final location after the displacement. Interestingly there is no indication of any existing defect under the islands. This indicates that the droplet may have formed by a random encounter of Sn atoms forming a cluster of critical size to act as a nucleus, or that the defects at which nucleation takes place are too small to be visible in large area scans. 
Such small defects must be single (or few) atom S-vacancies, since metal-like defects influence the electronic configuration around them and are visible in this scan area sizes (Fig. 4.1c). As the surface coverage of the sample was considerably below $0.1 \mathrm{ML}$ it is hard to estimate any distribution parameters, due to the scarce presence of the droplets in the first place.

To stimulate diffusion on the surface, the sample was heated up to the temperature of $200-300^{\circ} \mathrm{C}$, using radiation from a resistively heated tungsten filament on the back of the sample for $1 \mathrm{~h}$. As the result of such treatment, we observed droplets of Sn etching the surface of $\mathrm{MoS}_{2}$ (see Fig. 4.3). Two kinds of etched shapes were found - a hole and a trench - on different locations on the surface. The trenches were etched by droplets from the side of the step edge. Holes were formed by Sn droplets situated on flat terraces, i.e. away from the step edges of $\mathrm{MoS}_{2}$. The etched shapes are strongly anisotropic and presumably follow the crystal orientation of $\mathrm{MoS}_{2}$, which can be seen by (i) the triangular shape of the etched holes and (ii) the fact that the etched trenches run parallel with respect to each other. Several works on surface etching report on similar, triangular - like shapes on flat terraces, that reflect the symmetry of $\mathrm{MoS}_{2}$ lattice and correspond to low-energy edge terminations ( $\mathrm{S}$ - edge or Mo - edge) [8][9]. While the nanoscale lateral etching of $\mathrm{MoS}_{2}$ layers is not explored deeply [9], similar trenches have also been observed in other systems [9][10][11]. Such trenches follow the crystal orientation of the substrate along the path with the lowest etch resistance [10]. It is worth noting that the trenches observed in our experiment do not strictly follow one crystal direction. 

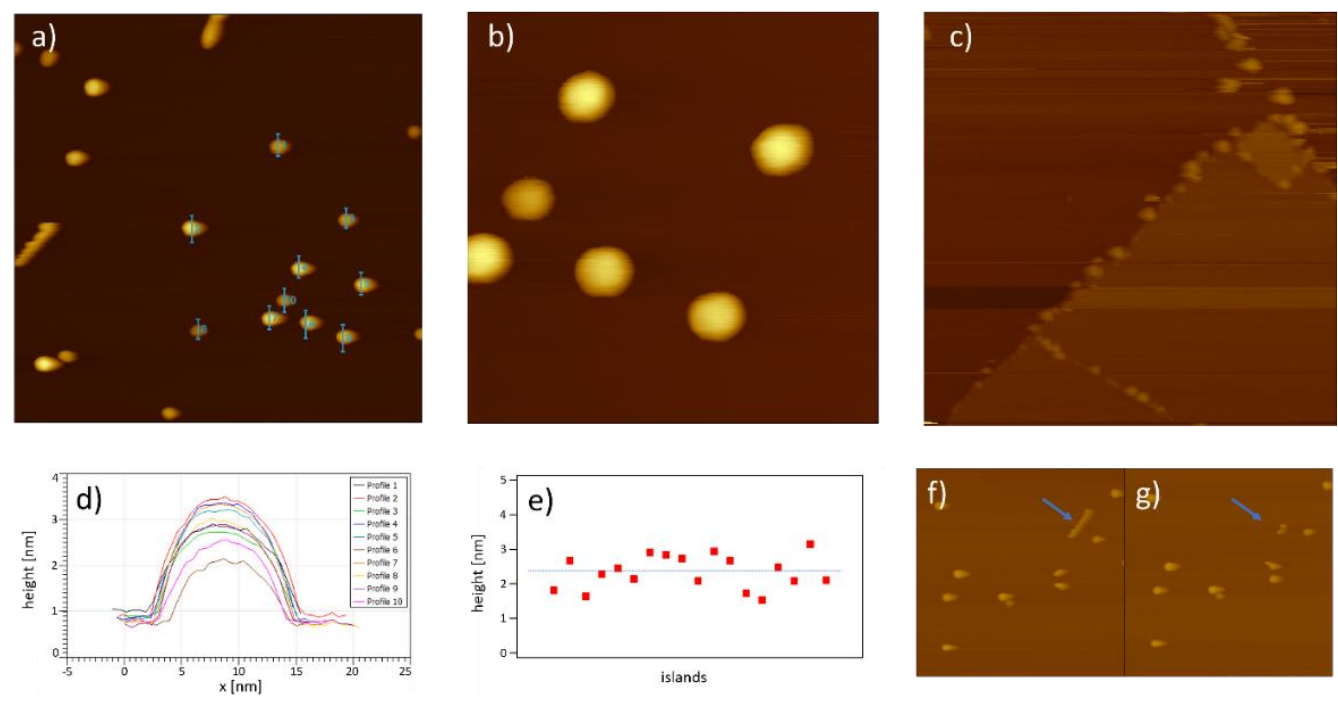

Fig. 4.2: Sn droplets on the surface of $\mathrm{MoS}_{2}$. a) STM images showing the distribution of the droplets on the surface of flat terraces. Note that the shapes of the droplets are distorted by the scan speed of the tip. b) The image shows a clear dome-like shape of the Sn droplets. c) Accumulation of $\mathrm{Sn}$ droplets along step edges. d) The average height is between $2-3 \mathrm{~nm}$, while the diameter is between $10-15 \mathrm{~nm}$. Different droplets have similar height to width ratio. e) The height distribution of droplets from (a) shows a large deviation from the mean value of $2.3 \AA$. (f-g) interaction with the STM tip causes islands to move. a) $\left.300 \times 300 \mathrm{~nm}^{2}, \mathrm{~V}=2.0 \mathrm{~V}, \mathrm{I}_{\mathrm{stp}}=0.1 \mathrm{nA} \mathrm{b}\right) 100 \times 100 \mathrm{~nm}^{2}, \mathrm{~V}=2.0 \mathrm{~V}, \mathrm{I}_{\mathrm{stp}}=0.1 \mathrm{nA} \mathrm{c)} 200 \times 200$ $\left.\mathrm{nm}^{2}, \mathrm{~V}=1.0 \mathrm{~V}, \mathrm{I}_{\mathrm{stp}}=0.1 \mathrm{nA} \mathrm{f}-\mathrm{g}\right) 300 \times 300 \mathrm{~nm}^{2}, \mathrm{~V}_{\text {bias }}=2.0 \mathrm{~V}, \mathrm{I}_{\text {stp }}=0.1 \mathrm{nA}$.

The reason for that may be that in the case of $\mathrm{MoS}_{2}$ the lattice has hexagonal symmetry, hence there are 3 equivalent directions on the surface. Interestingly, longer annealing did not influence the lengths of the trenches nor the sizes of the holes. Hence, it can be concluded that it is a self-terminating process, as only the initial size of a droplet determined how much the droplet would etch the substrate (Fig. 4.3b). A simple explanation of this phenomenon is a saturation effect, where the etched material is incorporated into the, initially composed purely of Sn, droplet, until a certain concentration of Mo and S is reached. The mixture of $\mathrm{Sn}$ and $\mathrm{MoS}_{2}$ can form a nonstoichiometric so-called Chevrel phase [12], described as $\mathrm{Sn}_{x} \mathrm{Mo}_{6} \mathrm{~S}_{8}$ with $\mathrm{x}$ usually between $0.5-2.0$ [13]. However, such compound is produced in much higher 
temperature and pressure, as well as in much larger time scales [14]. Therefore, the droplets accumulated in the trenches may simply be a disordered mixture of the three elements.
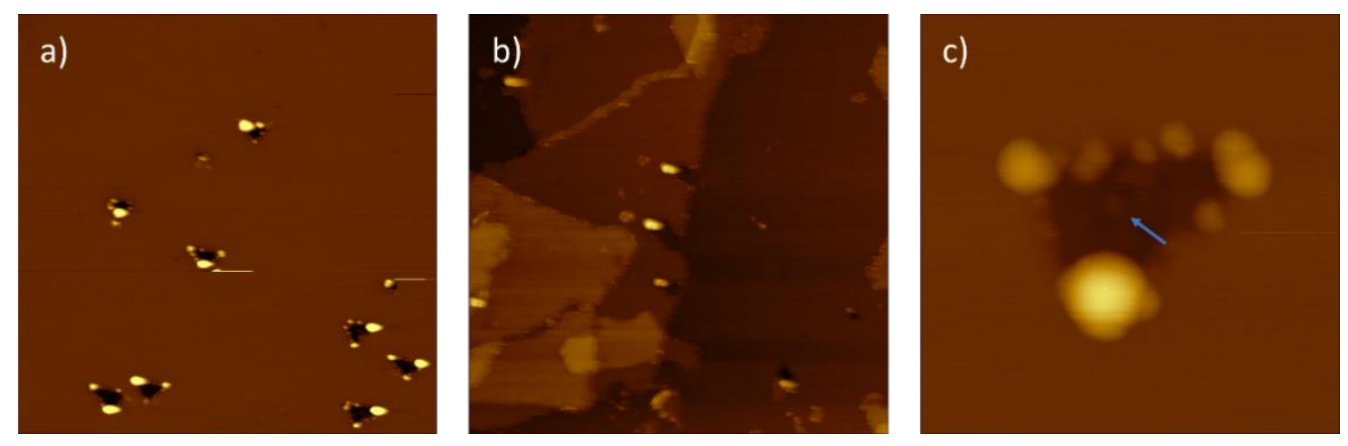

Fig. 4.3: Sn droplets etch the $\mathrm{MoS}_{2}$ surface. One can distinguish 2 kinds of etching-related structures on the $\mathrm{MoS}_{2}$ surface, a hole (a) and a trench (b). Holes of roughly triangular shape appear on flat surface areas, suggesting that the substrate surface is etched along the symmetry directions. When the droplets are located at step edges, they etch trenches in $\mathrm{MoS}_{2}$ flakes. c) When a droplet is big enough, STM scans inside it reveal that deeper layers of the $\mathrm{MoS}_{2}$ are being etched (marked by the blue arrow). a) $300 \times 300 \mathrm{~nm}^{2}, V_{\text {bias }}=1.0 \mathrm{~V}, I_{\text {stp }}=0.1 \mathrm{nA}$ b) $50 \times 50 \mathrm{~nm}^{2}, V_{\text {bia s }}=1.0 \mathrm{~V}, I_{\text {stp }}=0.1 \mathrm{nA}$ c) $300 \times 300 \mathrm{~nm}^{2}, V_{\text {bias }}=1.0 \mathrm{~V}, I_{\text {stp }}=0.1 \mathrm{nA}$.

As the enhanced diffusion did not bring about any desired change to the structure of $\mathrm{Sn}$, we decided to turn the other way and decrease the sample temperature during the deposition. Decreased temperature would limit the diffusion of Sn atoms and thereby hamper forming disordered islands, while metastable ordered phases may result. In the first experiment described above the Sn source was located close to the sample (the separation between the tip of the source and the sample was about $5 \mathrm{~cm}$ ), effectively heating it up during the deposition. Since the setup does not allow for active sample cooling during deposition, we decided to significantly increase the Sn source-sample distance and repeat the experiment. As the distance increased by over $30 \mathrm{~cm}$, we assumed the sample is at (or at least close to) room temperature (RT) during deposition.

Deposition at RT with increased source-sample distance indeed resulted in formation of ordered Sn islands with clearly anisotropic crystalline shape (see Fig. 4.4). The islands 
are oriented with respect to high symmetry directions. Many of them are approximately rectangular and very flat. The average heigth of the islands decreased considerably with respect to the previous experiment, and deviates less from the average value of $1.7 \AA$. The islands have a lateral size of several tens of $\mathrm{nm}$. The rectangular shape of the islands could be explained by $\mathrm{Sn}$ forming $\beta$-Sn, which has tetragonal crystal structure. Unfortunately $\beta-\mathrm{Sn}$ is a metalic allotrope of $\mathrm{Sn}$, which does not have any exotic (topological) properties, predicted for the monolayer of $\alpha-\mathrm{Sn}$ - the diamond-like $\mathrm{Sn}$ allotrope, which in its monolayer form has a graphene-like honeycomb lattice. In bulk, $\beta$-Sn is energetically favorable at higher temperature, while $\alpha$-Sn becomes the dominant allotrope at the transition temperature of $13^{\circ} \mathrm{C}$ [15]. The process of $\beta \Rightarrow>\alpha$ transition is known as "Sn pest". The phase diagram in the $2 \mathrm{D}$ regime is unknown and should be strongly substrate dependent, however, based on the overall trend of the bulk phase diagram, we expect that further decrease of the temperature might promote growth of $\alpha$-Sn. This hypothesis finds it confirmation in a few reports on stanene, where deposition on a low-temperature substrate was resulted in obtaining the $2 \mathrm{D}$ material [16][17][18][19]

Finally, similarly to the dome-shaped droplets, these ordered Sn structures are also easily displaced by the STM tip (see Fig 4.4f-i). Displacement by forces as weak as the one exerted by the STM tip suggests very weak interaction with the substrate. This is a promising result as weakly interacting substrates are the desired ones for epitaxial growth of stanene. 


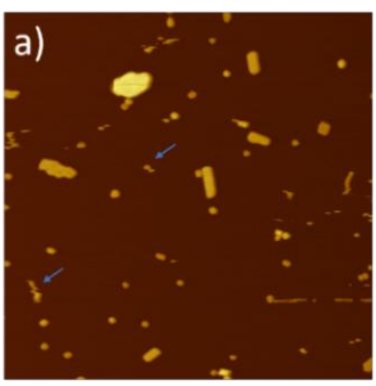

d)
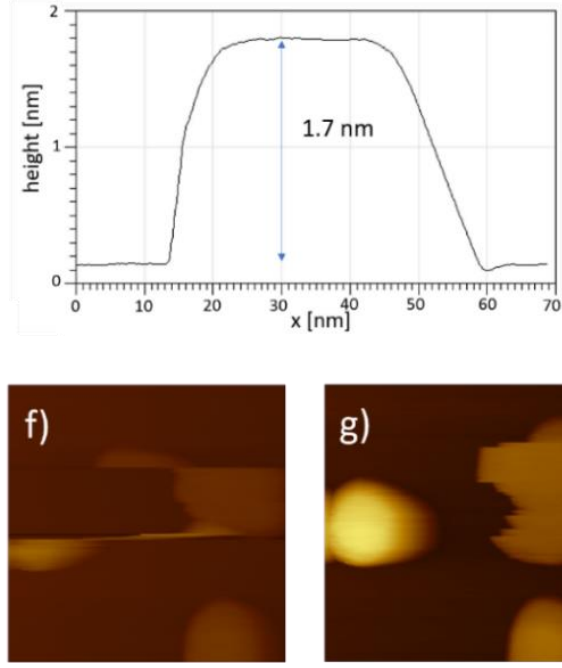
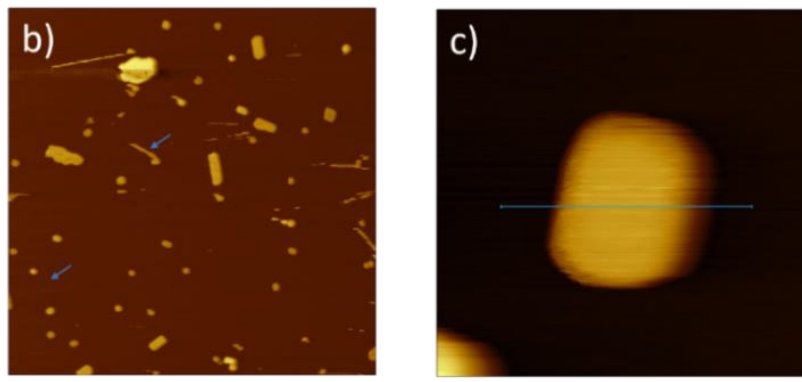

e)
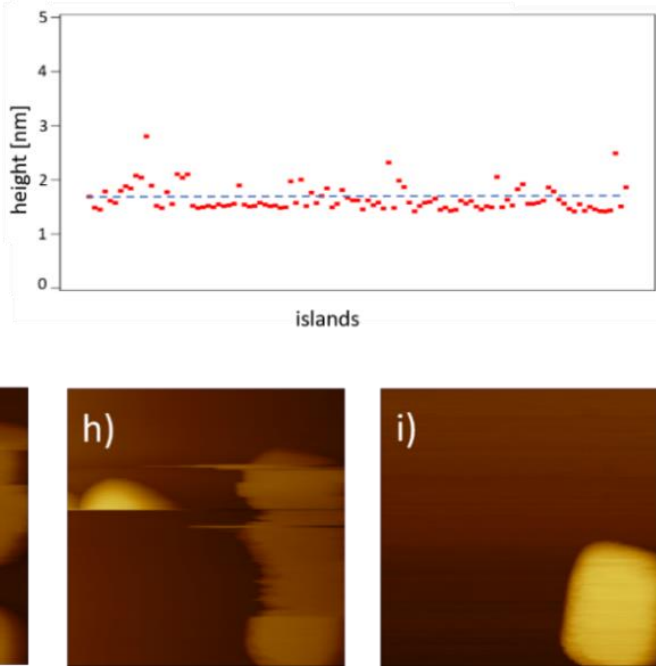

Fig 4.4: Sample surface after Sn deposition at RT. (a-b) consecutive STM images showing Sn islands. The comparison between a) and b) shows that the islands move on the surface marked by the blue arrows. c) STM image of a rectantangular Sn island with a marked line profile shown in d). Note that the flat top of the island, as well as the straight edges, are indicative of an ordered, crystalline structure. (e) Height diagram showing the average height of a large number of islands in (a) marked by blue line at $1.7 \AA$. f-i) consecutive STM images showing displacement of islands by the STM tip. a-b) $500 \times 500 \mathrm{~nm}^{2}, \mathrm{~V}_{\text {bias }}=1.2 \mathrm{~V}, \mathrm{I}_{\text {stp }}=0.1 \mathrm{nA} \mathrm{c)} 100 \times 100 \mathrm{~nm}^{2}, \mathrm{~V}_{\text {bias }}=1.0 \mathrm{~V}$, $\mathrm{I}_{\text {stp }}=0.1 \mathrm{nA} \mathrm{f-i)} 100 \times 100 \mathrm{~nm}^{2}, \mathrm{~V}_{\text {bias }}=0.8 \mathrm{~V}, \mathrm{stp}=0.1 \mathrm{nA}$ 


\subsection{Conclusions}

The experiment aimed at forming a honeycomb monolayer of $\mathrm{Sn}$ - stanene - on a bulk $\mathrm{MoS}_{2}$ sample. While this primary goal has not been achieved, we found clues that may direct future work. The initial, expected advantage of the $\mathrm{MoS}_{2}$ substrate was confirmed as the interactions with the deposited Sn formations were weak enough to be disrupted by the STM forces, resulting in displacement of islands upon measurement. This property of the substrate resulted, however, in the formation of disordered - droplet like structures. Further annealing the system resulted in etching of the $\mathrm{MoS}_{2}$ surface by these Sn droplets, in a self-terminating process. To obtain ordered structures we needed to decrease the deposition temperature of the sample. This allowed us to reach the regime where an ordered $\mathrm{Sn}$ phase could form, however the exact phase diagram of $\mathrm{Sn}$ on $\mathrm{MoS}_{2}$ in the $2 \mathrm{D}$ regime is unknown. Additionally, the decrease in deposition temperature hampered the diffusion of $\mathrm{Sn}$ atoms which could also play a role in the formation an ordered phase. The obtained crystalline $\mathrm{Sn}$ formations are rectangular, indicating that they are $\beta$-Sn multilayer islands. While $\beta$-Sn is a metallic allotrope, not interesting in the context of honeycomb 2D materials, we confirmed the crucial idea of the feasibility of $\mathrm{MoS}_{2}$ to support ordered Sn layers in the nanoscale regime. The possible solution to the encountered obstacles could be a cold sample deposition. Decreasing the temperature even further would further decrease the diffusion rate of Sn atoms, and make the $\alpha$-Sn allotrope energetically favorable

\section{References}

[1] W. Xiong et al., "Band engineering of the MoS2/stanene heterostructure: strain and electrostatic gating," Nanotechnology, vol. 28, no. 19, p. 195702, May 2017, doi: 10.1088/1361-6528/aa68d8.

[2] C.-C. Ren, Y. Feng, S.-F. Zhang, C.-W. Zhang, and P.-J. Wang, "The electronic properties of the stanene/MoS ${ }_{2}$ heterostructure under strain," RSC Adv., vol. 7, no. 15, pp. 9176-9181, 2017, doi: 10.1039/C6RA26169H.

[3] D. Liang et al., "Tunable band gaps in stanene/MoS2 heterostructures," J. Mater. Sci., vol. 52, no. 10, pp. 5799-5806, May 2017, doi: 10.1007/s10853-0170817-z. 
[4] L. Zhang et al., "Structural and Electronic Properties of Germanene on MoS 2," Phys. Rev. Lett., vol. 116, no. 25, p. 256804, Jun. 2016, doi:

10.1103/PhysRevLett.116.256804.

[5] R. van Bremen, Q. Yao, S. Banerjee, D. Cakir, N. Oncel, and H. J. W. Zandvliet, "Intercalation of Si between $\mathrm{MoS}_{2}$ layers," Beilstein J. Nanotechnol., vol. 8, pp. 1952-1960, Sep. 2017, doi: 10.3762/bjnano.8.196.

[6] R. Addou, L. Colombo, and R. M. Wallace, "Surface Defects on Natural MoS 2," ACS Appl. Mater. Interfaces, vol. 7, no. 22, pp. 11921-11929, Jun. 2015, doi: 10.1021/acsami.5b01778.

[7] P. Bampoulis, R. van Bremen, Q. Yao, B. Poelsema, H. J. W. Zandvliet, and K. Sotthewes, "Defect Dominated Charge Transport and Fermi Level Pinning in MoS ${ }_{2}$ /Metal Contacts," ACS Appl. Mater. Interfaces, vol. 9, no. 22, pp. 19278-19286, Jun. 2017, doi: 10.1021/acsami.7b02739.

[8] M. Yamamoto, T. L. Einstein, M. S. Fuhrer, and W. G. Cullen, "Anisotropic Etching of Atomically Thin MoS 2," J. Phys. Chem. C, vol. 117, no. 48, pp. 2564325649, Dec. 2013, doi: 10.1021/jp410893e.

[9] Z. Wang et al., "Controllable etching of MoS2 basal planes for enhanced hydrogen evolution through the formation of active edge sites," Nano Energy, vol. 49, pp. 634-643, Jul. 2018, doi: 10.1016/j.nanoen.2018.04.067.

[10] R. van Bremen et al., "Ge ${ }_{2}$ Pt hut clusters: A substrate for germanene," $J$. Appl. Phys., vol. 124, no. 12, p. 125301, Sep. 2018, doi: 10.1063/1.5046997.

[11] L. C. Campos, V. R. Manfrinato, J. D. Sanchez-Yamagishi, J. Kong, and P. Jarillo-Herrero, "Anisotropic Etching and Nanoribbon Formation in Single-Layer Graphene," Nano Lett., vol. 9, no. 7, pp. 2600-2604, Jul. 2009, doi: 10.1021/n1900811r.

[12] R. CHEVREL, M. SERGENT, and J. PRIGENT, "Sur de Nouvelles Phases Sulfurees Ternaires du Molybdene,” J. SOLID STATE Chem., vol. 3, pp. 515-519, 1971.

[13] H. A. Wagner, "STOICHIOMETRY OF THE CHEVREL PHASE SnMo,\&," p. 5 . 
[14] J. M. Tarascon, F. J. Disalvo, D. W. Murphy, G. W. Hull, E. A. Rietman, and J. V. Waszczak, "Stoichiometry and physical properties of ternary molybdenum chalcogenides MxMo6X8 (X= S, Se; M = Li, Sn, Pb)," J. Solid State Chem., vol. 54, no. 2, pp. 204-212, Sep. 1984, doi: 10.1016/0022-4596(84)90147-6.

[15] Reginald W. Smith, "The alpha(semiconductor) $<=>$ beta(metal) transition in tin," J. -Common Met., vol. 114, pp. 69-80, 1986.

[16] Y.-H. Song et al., "High-buckled $\sqrt{3} \times \sqrt{3}$ stanene with topologically nontrivial energy gap," p. 14, 2017.

[17] Y. Zang et al., "Realizing an Epitaxial Decorated Stanene with an Insulating Bandgap," Adv. Funct. Mater., vol. 28, no. 35, p. 1802723, 2018, doi: 10.1002/adfm.201802723.

[18] M. Liao et al., "Superconductivity in few-layer stanene," Nat. Phys., vol. 14, no. 4, pp. 344-348, Apr. 2018, doi: 10.1038/s41567-017-0031-6.

[19] J. Deng et al., "Epitaxial growth of ultraflat stanene with topological band inversion," Nat. Mater., vol. 17, no. 12, pp. 1081-1086, Dec. 2018, doi: 10.1038/s41563-018-0203-5. 



\section{CHAPTER 5}

\section{Li intercalation into multilayer graphene with controlled defect densities}

\subsection{Introduction}

Since its discovery in 2004 [1], the novel two-dimensional material graphene holds great promise in a wide spectrum of research and industrial fields, due to its unique electronic structure and extraordinary physical properties. Several top-level review articles thoroughly summarize the hallmarks of graphene [2][3][4][5]. With its very large surface-to-volume ratio [6], high electrical and thermal conductivity [1][7], graphene holds considerable promise as a new anode material in energy-storage devices, such a $\mathrm{Li}$ - ion batteries and supercapacitors [8][9]. The performance of the electrodes in such devices is dependent on their surface area, which should be as high as possible, and charge separation at the electrode-electrolyte interface. Graphene is a very good material choice for this application, offering high intrinsic electrical conductivity, good resistance to oxidative processes and high temperature stability. During the synthesis of graphene, structural defects might be introduced, for example in the form of carbon vacancies. Several DFT calculations suggest that defects enhance the lithiation and intercalation process in single and multilayer graphene systems [10][11][12]. In this article we report an investigation of the role of such vacancies on the process of $\mathrm{Li}$ intercalation in multilayer graphene grown by chemical vapor deposition (CVD) on $\mathrm{Ni}$ substrates. 


\subsection{Methods}

Sample preparation as well as measurements were done in our home labs. The samples consisted of multilayer graphene flakes on polycrystalline Ni thin films, grown by CVD. The graphene growth mechanism on $\mathrm{Ni}$ catalysts includes three main steps: (i) catalytic decomposition of precursor molecules to produce $\mathrm{C}$ atoms, (ii) carbon dissolution into the metal at high temperatures and (iii) crystallization of carbon atoms onto the metal surface to form graphene. Due to the relatively high solubility of graphene in $\mathrm{Ni}$ at the elevated temperatures required for catalytic decomposition of precursor molecules, a significant concentration of $\mathrm{C}$ atoms can be absorbed in the Ni film. Upon cooling, the dissolved carbon segregates back to the surface and forms a graphene multilayer. In order to investigate the $\mathrm{Li}$ intercalation process, $\mathrm{Li}$ atoms were evaporated on top of previously synthesized multilayer-graphene/ $\mathrm{Ni}(200 \mathrm{~nm}) / \mathrm{SiO}_{2}(300 \mathrm{~nm}) / \mathrm{Si}$ (substrate) samples from a Li dispenser (SAES Getters) in ultra-high vacuum. The evaporation occurs upon heating the $\mathrm{Li}$ containing compound inside the $\mathrm{Li}$ dispenser by applying an electric current through it. Various heating currents were tested to define the current level enabling a stable and controllable evaporation rate (keeping the evaporation time fixed to $5 \mathrm{~min}$ ). The sample was annealed with a resistive heating system up to $300^{\circ} \mathrm{C}$. An ion implanter IMC-200 from Ion Beam Services was used to implant Ar ions at the energy of $190 \mathrm{keV}$. Raman spectroscopy was performed using a WiTec confocal Raman system. The laser wavelength used for the measurements was $532 \mathrm{~nm}$. XPS measurements were performed with a hemispherical analyzer (SPECS PHOIBOS 100) and an X-ray gun with $\mathrm{Mg}$ and $\mathrm{Al}$ anodes. The XPS measurements were acquired using Mg K-alpha X-rays.

\subsection{Results and discussion}

Fig. 5.1 shows the characterization of a graphene/Ni/SiO2/Si(111) sample by means of Raman spectroscopy. Fig. 5.1a shows a Raman map of the integrated intensity of the 2D peak region $\left(2550-2840 \mathrm{~cm}^{-1}\right)$. The shapes of the thickest flakes can be easily seen. Fig. $5.1 \mathrm{~b}$ is a Raman spectrum recorded on the point marked with the blue dot in Fig 5.1a. It can be seen that the $\mathrm{D}$ peak $\left(1350 \mathrm{~cm}^{-1}\right)$ corresponding to defects in the graphene layers is essentially invisible, which indicates that the graphene has a low intrinsic defect density. The intensity of the $G$ peak $\left(1583 \mathrm{~cm}^{-1}\right)$ compared to that of the 2D peak $\left(2710 \mathrm{~cm}^{-1}\right)$ indicates that this particular flake is more than 5 layers thick. 
To observe the process of intercalation of $\mathrm{Li}$ ions between graphene layers, we utilized photoelectron spectroscopy (XPS). A direct observation of the Li $1 s$ peak is very difficult using our system, due to the neighbouring Ni $3 p$ peak (its satellite peak overlaps with the Li $1 s$ signal since non-monochromatic X-rays are used) and the very low photoionization cross section, which makes the Li peak virtually undetectable.
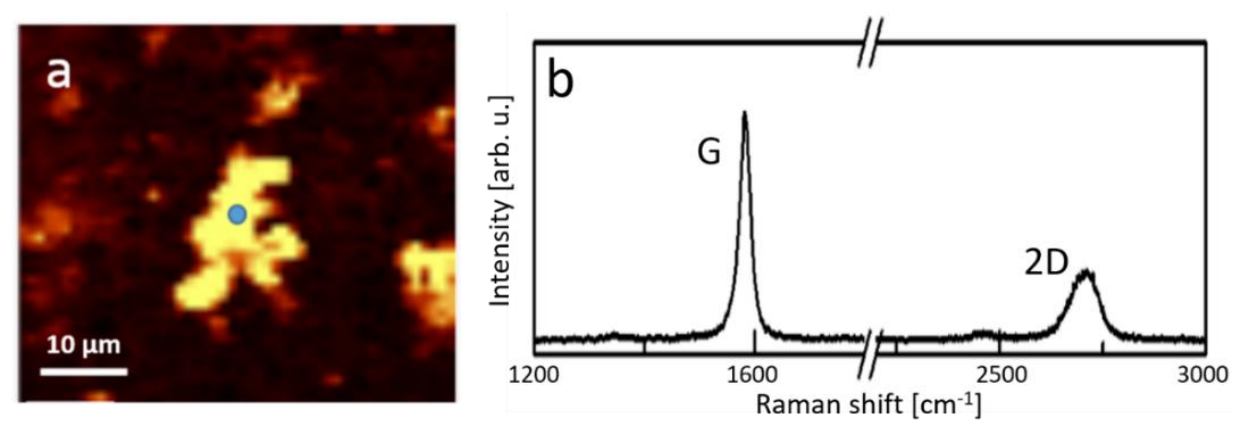

Fig. 5.1: (a) A Raman map showing the integrated intensity of the $2 \mathrm{D}$ peak region $\left(2550-2840 \mathrm{~cm}^{-1}\right)$ (b) Raman spectrum measured on the point marked with blue dots in (a).

However, the amount of deposited Li can be determined by the suppression of substrate core level peaks due to inelastic scattering of photoelectrons at Li ions. It can be quantified as follows - if $I_{0}$ is the initial intensity of the signal from a core level in an XPS measurement, after deposition of an additional layer of some material the intensity $I$ will change according to: $I=I_{0} e^{-\frac{t}{\lambda}}$, where $t$ is the thickness of the new layer and $\lambda$ is the inelastic mean free path of photoelectrons in the deposited material. This formula allows for an estimate of the thickness of a deposited layer $t$ for perfectly layered samples, according to $t=\ln \left(I / I_{0}\right) \lambda$. Since our system is not perfectly layered, we can only extract an "effective thickness", which can be used to study the intercalation process by comparing effective thicknesses measured for different elements in the system. This holds because the two elements for which we compare the signals, $\mathrm{C}$ and $\mathrm{Ni}$, are separated and do not mix, i.e. the multilayer graphene resides on top of the $\mathrm{Ni}$ layer. Moreover, while Li ions can intercalate into the graphene multilayers, diffusion of Li ions into the $\mathrm{Ni}$ substrate is negligible [13]. Therefore, the deposition as well as intercalation processes were observed via C $1 s, \mathrm{Ni} 2 p$ and Ni $3 p$ core level peaks. Figure 5.2 illustrates this for as-grown multilayer graphene on $\mathrm{Ni}$. 
In case of $\mathrm{Ni}$, the only observed phenomenon was the peak intensity suppression (Fig. 5.2 b, c). Using $\lambda_{N i 2 p}=20 \AA$ and $\lambda_{N i 3 p}=47 \AA$ [14] (The kinetic energy $E_{k}$ of Ni $2 p$ and Ni $3 p$ photoelectrons was set to $401 \mathrm{eV}$ and $1187 \mathrm{eV}$, respectively) the effective thickness was calculated (Fig. 5.2d). The reason for measuring two peaks of the same element was to minimize any influence of errors in the estimate of the inelastic mean free path on the peak intensity change. We note that the two calculated values may differ significantly, in particular the spread in Ni $3 p$ peak intensity suppressions for similar $\mathrm{Li}$ coverage was large compared to that of Ni $2 p$ peaks. This is most probably caused by a larger error in determining the $\mathrm{Ni} 3 p$ peak intensity, due its low overall signal to background ratio. The effect of $\mathrm{Li}$ deposition on the $\mathrm{C} 1 s$ core level is more complex. Apart from intensity suppression $\left(\lambda_{C l s}=40 \AA\right.$ [14], $\left.E_{k}=969 \mathrm{eV}\right)$ we observed a shift towards higher binding energy and a tail on the high binding energy side of the peak (Fig. 5.2a). The intercalation process takes place via ionic diffusion, which is presumably fast for the displacement of ions in between graphene sheets. Since a perfect graphene sheet is impermeable for ions, diffusion in the out-of-plane direction may occur through defects in graphene layers.

Access to deeper layers may also be provided by flake edges. The main indicators of $\mathrm{Li}$ intercalation in XPS measurements are (i) an energy shift of the $\mathrm{C} 1 s$ peak relative to its original position, as well as (ii) the difference between the effective Li thickness values determined from the suppression of $\mathrm{Ni}$ and $\mathrm{C}$ core level peaks. The shift is associated with the formation of $\mathrm{LiC}_{6}$ rings and caused by charge transfer between $\mathrm{Li}$ ions and $\mathrm{C}$ atoms, where the electrons transferred from Li fill the $\pi$ bands of graphene [15][16]. The difference in suppression is related to the high surface sensitivity of the XPS technique. Top layers of the sample contribute to the overall signal to a much higher degree than sub-surface layers. When $\mathrm{Li}$ intercalates into multilayer graphene, it "uncovers" top layers. As a result, the effective thickness of the $\mathrm{Li}$ "layer" on/in multilayer graphene, measured from the suppression of the photoelectron signal, will be smaller than as the case for the underlying Ni substrate, for which no Li in-diffusion occurs. By comparing the suppression of $\mathrm{Ni}$ and $\mathrm{C}$ core level peaks in the form of effective thickness, we can tell if $\mathrm{Li}$ intercalated or was removed (e.g. by surface diffusion or sublimation upon annealing) (Fig 5.2d). 
a)

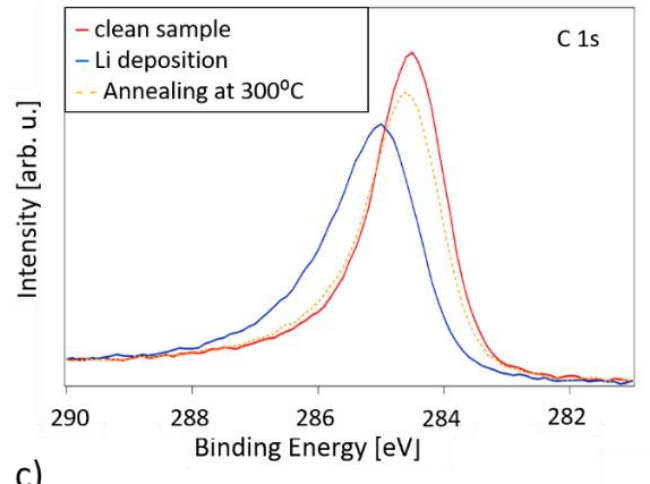

c)

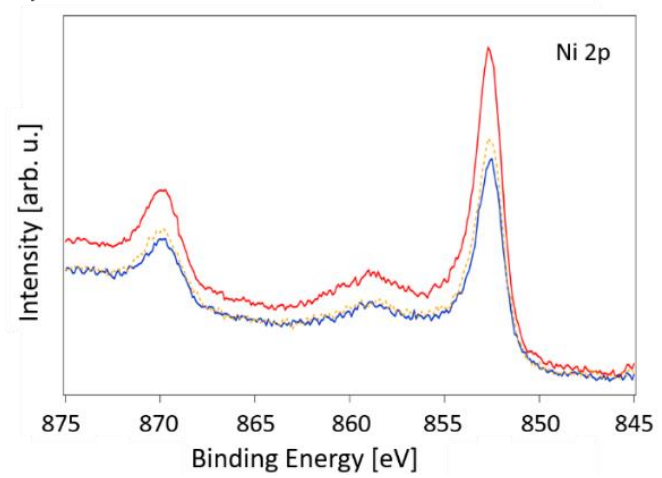

b)
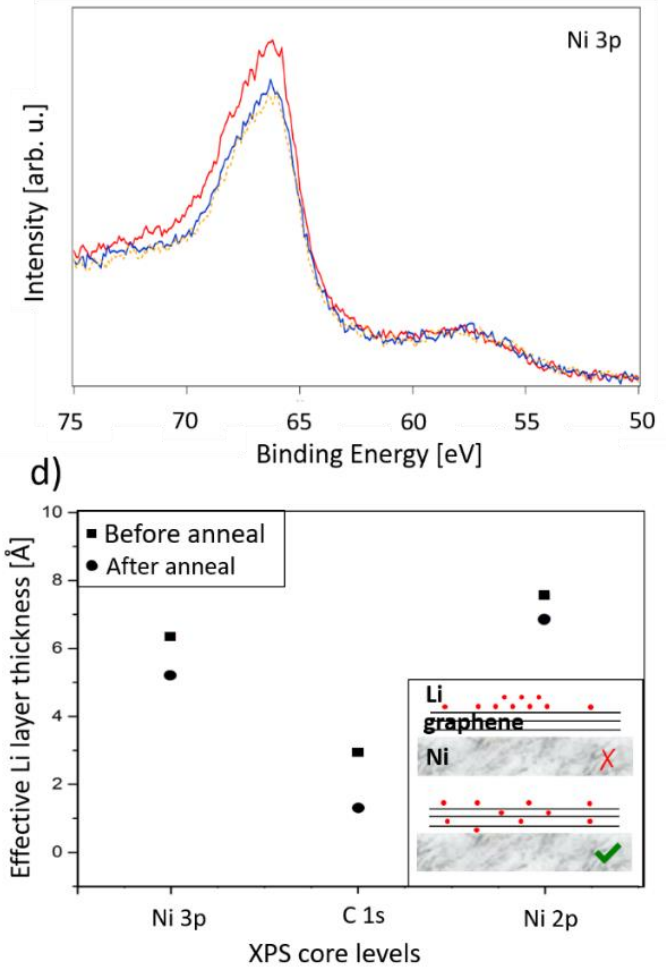

Fig. 5.2: Evolution of $\mathrm{C} 1 s$ (a), $\mathrm{Ni} 3 p$ (b) and $\mathrm{Ni} 2 p$ (c) core level peaks upon deposition of $\mathrm{Li}$ and subsequent annealing. All peaks are suppressed as a result of Li deposition, due to inelastic scattering of photoelectrons by $\mathrm{Li}$ ions. The shift towards higher binding energy and the tail on the higher binding energy side of the $\mathrm{C} 1 s$ peak is the result of charge transfer between $\mathrm{C}$ and $\mathrm{Li}$ atoms upon deposition. Annealing causes recovery of the $\mathrm{C} 1 s$ peak to its original position, and a general recovery of the intensity in all 3 measured peaks. d) Comparison of the effective thicknesses of the Li layer before and after 5 mins of annealing at $300^{\circ} \mathrm{C}$. The $\mathrm{C} 1 s$ signal is much less suppressed which indicates intercalation between graphene layers, as shown in the inset. A general smaller drop of the effective thickness for all core levels after annealing suggests partial removal or sintering of Li material. 
Diffusion and intercalation processes are thermally activated, and some reports suggest that annealing enhances the process of $\mathrm{Li}$ intercalation into graphene[17]. Therefore, after deposition we annealed the sample at $300^{\circ} \mathrm{C}$ for 5 mins. Fig 5.2 shows the effect of annealing on all the measured peaks as well as on the calculated effective thickness of Li layer. We observed a shift back to its original position of the $\mathrm{C} 1 s$ core level peak, as well as a general decrease in effective thickness measured on all 3 core level peaks. From these two effects, we conclude that annealing in fact de-intercalates $\mathrm{Li}$ and partially removes it from the sample surface.

We now turn to the main goal of the experiment, i.e. to investigate how the defect density in multilayer graphene affects the $\mathrm{Li}$ intercalation process. The defects were imposed by Ar ion irradiation. An ion beam energy of $190 \mathrm{keV}$ was chosen in order to implant deep into the Ni substrate. In that way, we made sure that no Ar signals could contribute to the core level measurements. Exposure to ion bombardment, with doses of $10^{12}, 10^{13}$ and $10^{14}$ ions $/ \mathrm{cm}^{2}$, resulted in various defect densities. Fig. 5.3 shows Raman spectra of a pristine sample as well as damaged multilayer graphene. These spectra are compared with spectra published by Martins Ferreira et al. [18]. In their report, a much lower ion energy of $90 \mathrm{eV}$ was used to study monolayer (M), bilayer (B) and trilayer (T) graphene samples, as well as highly oriented pyrolytic graphite (HOPG). In spite of these differences, the obtained Raman spectra are surprisingly similar. The average inter-defect distances are consequently estimated to be of the same order of magnitude as those in Ref. [18], i.e. between 10 and $1 \mathrm{~nm}$ respectively for doses between $10^{12}$ and $10^{14}$ ions $/ \mathrm{cm}^{2}$. It can thus be concluded that we obtained lightly to heavily damaged multilayer graphene.

In order to investigate the influence of the inflicted damage on the intercalation process, a series of Li depositions was performed. For better comparison, as well as to eliminate the uncertainty coming from the source stability, next to each damaged graphene we mounted pristine samples as reference. In that way we could directly compare the behaviour with respect to pristine graphene. Fig. 5.4a-c show the behaviour of $\mathrm{C} 1 \mathrm{~s}$ core level peak maximum shift upon consecutive depositions and final anneal for samples with different defect densities. All variations are within error bars and the behaviour is virtually the same for all 3 examined defect densities and there are no visible trends with respect to defect density. Fig. 5.4d compares the behaviour for all examined samples in terms of effective thickness and the shift of peak maximum in the deposition sequence. For clarity we omitted the annealing step. The fact that both parameters are correlated 
in the same way for each irradiation dose indicates that the $\mathrm{Li}$ ions have similar concentration at similar depth. The depositions were done until saturation of the peak shift was observed, which we take to be indicative of saturation of the $\mathrm{Li}$ intercalation process.

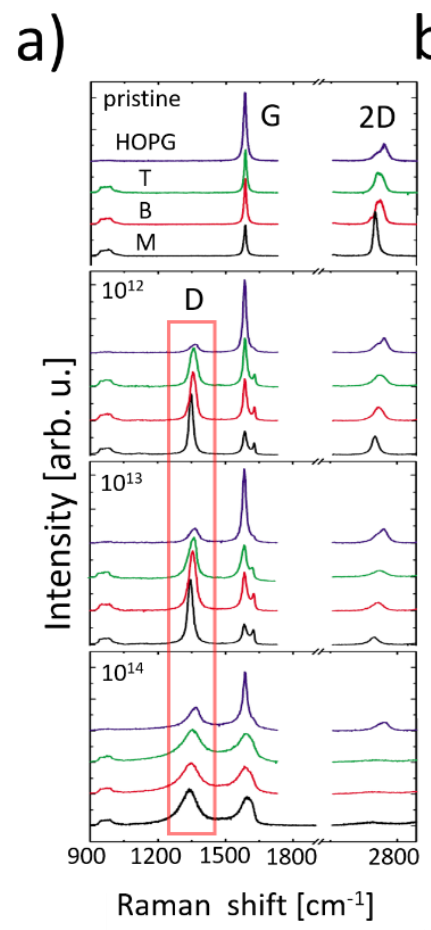

b)

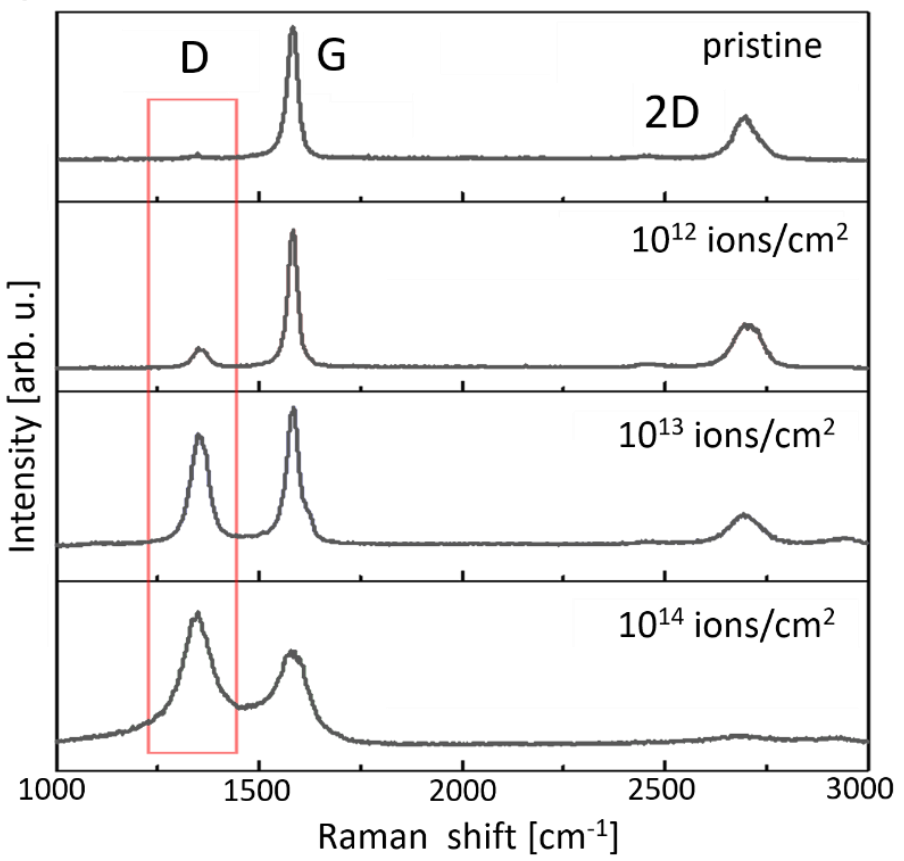

Fig. 5.3: Raman spectra of a pristine sample as well as samples irradiated with Ar-ions (b). The irradiated samples received different ion doses, as indicated. As a result, 3 different defect densities were obtained, as is evident from the different intensities of the defect peak D. The similarity with previously reported Raman spectra of damaged monolayer (M), bilayer (B) and trilayer (T) graphene and highly oriented pyrolytic graphite (HOPG) (a, adapted from Martins Ferreira et al. [18]) allows us to estimate defect densities to be also similar to those described in that report. 
a)

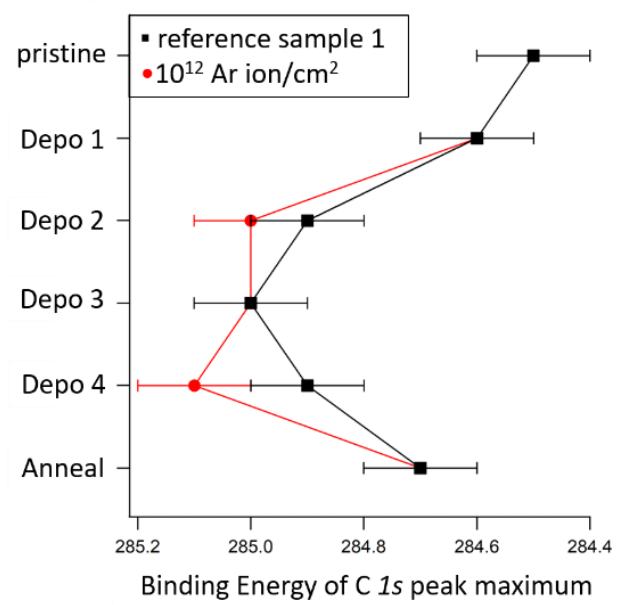

c)

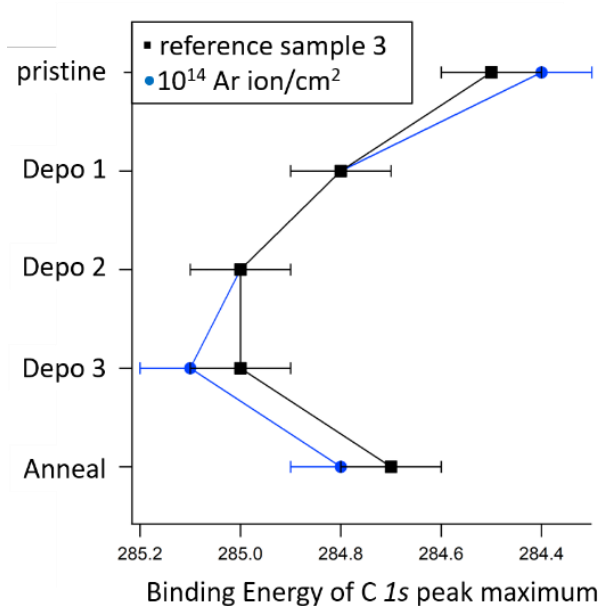

b)

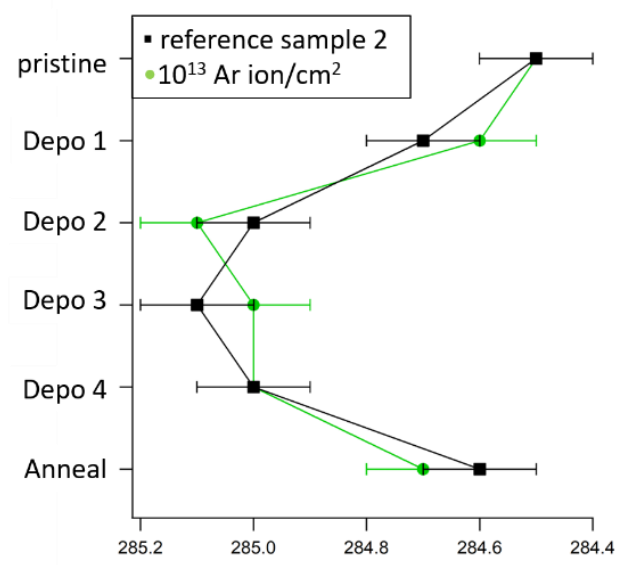

Binding Energy of $\mathrm{C}$ 1s peak maximum

d)

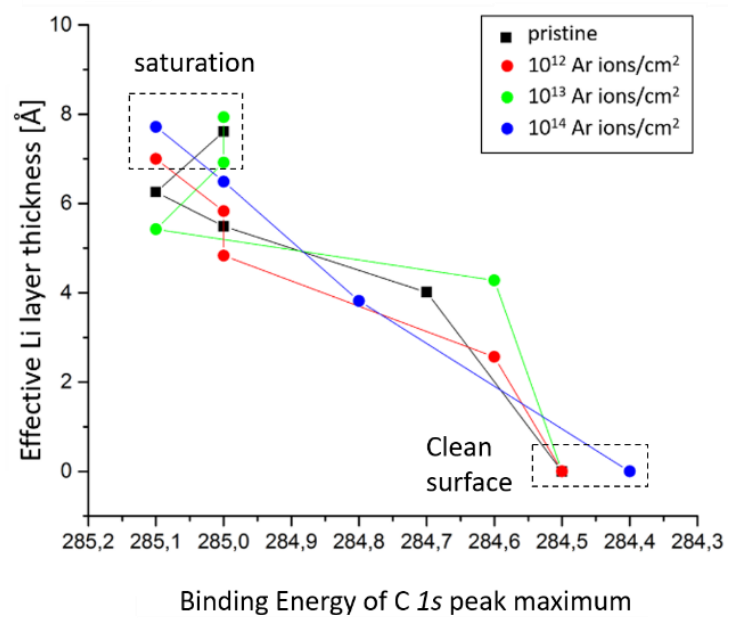

Fig. 5.4: a-c) a C $1 s$ core level peak maximum shift upon Li deposition for samples that were exposed to different Ar ion irradiation doses. The observed behaviour of the damaged graphene doesn't differ significantly from the reference pristine samples for any defect density examined. d) A comparison of the effect of consecutive Li depositions on C $1 s$ core level peak shift and its effective layer thickness. Each point in the graph represents one deposition. The "pristine" graph here is represented by reference sample 2, the other two reference samples were removed for clarity. Note that for heavily damaged graphene (dose of $10^{14} \mathrm{ions} / \mathrm{cm}^{2}$ ) the last deposition was $10 \mathrm{~min}$, hence there is one data point fewer. All 4 samples behave very similarly, within the accuracy of determining the peak shifts $(0.1 \mathrm{eV})$. 
Since a defect - free graphene sheet is impermeable, and the defects imposed by ion implantation appear to have no effect, a possible reason for such behaviour could stem from the fact that the multilayer graphene consists of flakes. In that scenario, $\mathrm{Li}$ intercalation is primarily facilitated by the edges of the flakes, where the edge density would be high enough for defects not to influence the intercalation ratio. Additional effects related to clogging of defects have been reported previously [19]. In the scenario proposed by that report, the Li ions tend to concentrate around defects and pose a steric hinderance to other $\mathrm{Li}$ ions. This constrains the lateral diffusion of the latter and screens the defects for them. This effective "clogging" effect may be an additional reason that suppresses any potential influence of defects on the intercalation process.

\subsection{Conclusions}

In conclusion, we performed a series of experiments to investigate the correlation between the $\mathrm{Li}$ intercalation process in multilayer graphene with controlled defect density. The results provide the insight that varying the defect density over several orders of magnitude has surprisingly little effect on the intercalation process. We mainly attribute this to intercalation being facilitated by the edges of the flakes (with lateral dimensions that are typically on the order of $10^{-5} \mathrm{~m}$ ), such that the edge density determines the intercalation rate for the whole range of defect densities studied. In addition, the accumulation of $\mathrm{Li}$ ions around defects, effectively clogging them, may further suppress their impact.

\section{References}

[1] K. S. Novoselov, "Electric Field Effect in Atomically Thin Carbon Films," Science, vol. 306, no. 5696, pp. 666-669, Oct. 2004, doi: 10.1126/science.1102896.

[2] A. H. Castro Neto, F. Guinea, N. M. R. Peres, K. S. Novoselov, and A. K. Geim, "The electronic properties of graphene," Rev. Mod. Phys., vol. 81, no. 1, pp. 109-162, Jan. 2009, doi: 10.1103/RevModPhys.81.109.

[3] C. N. R. Rao, A. K. Sood, K. S. Subrahmanyam, and A. Govindaraj, "Graphene: The New Two-Dimensional Nanomaterial," Angew. Chem. Int. Ed., vol. 48, no. 42, pp. 7752-7777, Oct. 2009, doi: 10.1002/anie.200901678. 
[4] J. Wintterlin and M.-L. Bocquet, "Graphene on metal surfaces," Surf. Sci., vol. 603, no. 10-12, pp. 1841-1852, Jun. 2009, doi: 10.1016/j.susc.2008.08.037.

[5] V. N. Kotov, B. Uchoa, V. M. Pereira, F. Guinea, and A. H. Castro Neto, "Electron-Electron Interactions in Graphene: Current Status and Perspectives," Rev. Mod. Phys., vol. 84, no. 3, pp. 1067-1125, Jul. 2012, doi:

10.1103/RevModPhys.84.1067.

[6] J. Xia, F. Chen, J. Li, and N. Tao, "Measurement of the quantum capacitance of graphene," Nat. Nanotechnol., vol. 4, no. 8, pp. 505-509, Aug. 2009, doi: 10.1038/nnano.2009.177.

[7] A. A. Balandin et al., "Superior Thermal Conductivity of Single-Layer Graphene," Nano Lett., vol. 8, no. 3, pp. 902-907, Mar. 2008, doi: 10.1021/n10731872.

[8] J. J. Yoo et al., "Ultrathin Planar Graphene Supercapacitors," Nano Lett., vol. 11, no. 4, pp. 1423-1427, Apr. 2011, doi: 10.1021/n1200225j.

[9] D. A. C. Brownson, D. K. Kampouris, and C. E. Banks, "An overview of graphene in energy production and storage applications," J. Power Sources, vol. 196, no. 11, pp. 4873-4885, Jun. 2011, doi: 10.1016/j.jpowsour.2011.02.022.

[10] D. Datta, J. Li, N. Koratkar, and V. B. Shenoy, "Enhanced lithiation in defective graphene," Carbon, vol. 80, pp. 305-310, Dec. 2014, doi: 10.1016/j.carbon.2014.08.068.

[11] L.-J. Zhou, Z. F. Hou, and L.-M. Wu, "First-Principles Study of Lithium Adsorption and Diffusion on Graphene with Point Defects," J. Phys. Chem. C, vol. 116, no. 41, pp. 21780-21787, Oct. 2012, doi: 10.1021/jp304861d.

[12] E. Lee and K. A. Persson, "Li Absorption and Intercalation in Single Layer Graphene and Few Layer Graphene by First Principles," Nano Lett., vol. 12, no. 9, pp. 4624-4628, Sep. 2012, doi: 10.1021/n13019164.

[13] T. B. Massalski, H. Okamoto, A. S. M. International, P. R. Subramanian, and L. Kacprzak, Binary Alloy Phase Diagrams, no. v. 3. ASM International, 1990.

[14] C. J. Powell and A. Jablonski, "NIST Electron Inelastic-Mean-Free-Path Database - Version 1.2, National Institute of Standards and Technology, Gaithersburg, MD (2010), . 
[15] G. K. Wertheim, P. T. Th. M. Van Attekum, and S. Basu, "Electronic structure of lithium graphite," Solid State Commun., vol. 33, no. 11, pp. 1127-1130, Mar. 1980, doi: 10.1016/0038-1098(80)91089-3.

[16] V. Z. Mordkovich, "Synthesis and XPS investigation of superdense lithiumgraphite intercalation compound, LiC2," Synth. Met., vol. 80, no. 3, pp. 243-247, Jul. 1996, doi: 10.1016/0379-6779(96)80209-8.

[17] L. Zhang, Y. Ye, D. Cheng, H. Pan, and J. Zhu, "Intercalation of Li at the Graphene/Cu Interface,” J. Phys. Chem. C, vol. 117, no. 18, pp. 9259-9265, May 2013, doi: 10.1021/jp401290f.

[18] E. H. Martins Ferreira et al., "Evolution of the Raman spectra from single-, few-, and many-layer graphene with increasing disorder," Phys. Rev. B, vol. 82, no. 12, p. 125429, Sep. 2010, doi: 10.1103/PhysRevB.82.125429.

[19] F. Yao et al., "Diffusion Mechanism of Lithium Ion through Basal Plane of Layered Graphene," J. Am. Chem. Soc., vol. 134, no. 20, pp. 8646-8654, May 2012, doi: 10.1021/ja301586m. 


\section{CHAPTER 6}

\section{Outlook}

The thesis shines light on possible research directions in the field of elemental 2D materials. In chapter 2 we concluded that $\mathrm{Li}$ intercalation in multilayer graphene on $\mathrm{Ni}$ thin films is not influenced by the defect density of the graphene sheets. The result is in accordance with previous experimental results for multilayer graphene, however it is also in contrast with calculations on monolayer graphene which suggest that higher defect density increases the lithiation capacity of graphene sheet. An important factor that is not addressed in the calculations is the existence of flakes' edges, which also allow for intercalation. The edges play even more important role in the $\mathrm{Li}$ intercalation process in the case of multilayer graphene - The flakes' edges area is bigger than the overall area of defects imposed on the flakes. An interesting idea would be to investigate when defect density starts to play a role in the intercalation process. This can be done by either changing the thickness of graphene flakes or by increasing their lateral dimensions.

In chapter 3 we presented the state of the art of epitaxial growth on stanene. We reviewed the field by collecting reports on theory and calculations regarding various stanene-substrate systems. Our review summarizes the main achievements in the field as well as points out substrate materials that have not yet been tried experimentally, but were tested theoretically in calculations. It is a good orientation point for newcomers to the field. The summary shows one more important issue - that none of the experimentally realized systems exhibits the theoretically predicted properties of freestanding stanene. In all cases the Sn monolayer is deformed with respect to the theoretical material and its properties are changed, due to interaction with the substrate. Hence the search for the "perfect" substrate remains is still ongoing. Here the 
straightforward proposition would be to try, in experiments, materials that have been calculated to support stanene. In chapter 5 we present our contribution to the search for suitable substrates. We chose $\mathrm{MoS}_{2}$ as the substrate due to the promising predicted properties of stanene- $\mathrm{MoS}_{2}$ heterostructures. The main of that work is that the temperature plays key role in the process of forming ordered Sn structures on the MoS2 surface. This opens the door for further exploration of the parameter space formed by the substrate temperature and deposition rate. A further decrease of the deposition temperature below room temperature, accompanied by an optimized deposition rate, might result in the formation of an ordered monolayer of Sn. It would be a big achievement in the field as the substrate is a semiconductor with a large bandgap while it is expected to interact weakly with the Sn adlayer due to it being a Van der Waals material.

Finally, in chapter 4 we presented an extensive investigation of the $\mathrm{Sn}-$ silicene $-\mathrm{ZrB}_{2}$ system. The experiments were part of a pioneering effort on investigating the interactions between two group IV materials in the $2 \mathrm{D}$ regime. The system has been proven to be rich and complex, with the formation of structures on the surface being heavily dependent on the annealing temperature. Strongly segregated phases as well as mixed Si/Sn phases were observed. The investigation still leaves a number of open questions.In particular, the effect of the deposition rate and the influence of the net amount of $\mathrm{Sn}$ on the structures formed upon post-deposition annealing are not fully explored. Regarding the obtained results, at low annealing temperatures, Sn can remove silicene from direct contact with $\mathrm{ZrB}_{2}$. This additional amount of $\mathrm{Si}$ forms additional layers on top of silicene. It may be worth investigation whether the formation obtained by deposition of Sn differs in some ways from the one obtained by deposition of additional Si. Furthermore, when optimized, Sn deposition can be a convenient method for patterning the sample surface. Further investigation is required to determine the origin of the LEED pattern when the amount of deposited material is above $1 \mathrm{ML}$.

In the case of the middle temperature regime, the origin of the new spots in LEED pattern is still not determined. Systematic measurements focused on this regime need to be conducted to solve that puzzle. Our hypothesis about the fluctuations of $\mathrm{Sn}$ atomic positions could be explored by low-temperature measurements e.g. STM at liquid helium temperature $(4 \mathrm{~K})$. 
The highest annealing temperature resulted in formation of an ordered layer of Sn on top of silicene. Measurements of the exact electronic properties of those Sn islands are still necessary as the already obtained STS measurements are not conclusive. In the case where the structure has a non-negligible bandgap, it would be an interesting system for further investigation in a more applied direction. The next step could be to use samples with $\mathrm{hBN}$ on top. Such samples have been already produced and allow for an effective encapsulation of the underlying silicene layer for several hours.

Deposition of Sn on that system may have two possible outcomes - either Sn intercalates below the hBN ( $\mathrm{Si}$ atoms have been shown to do that) or it stays on top. In the former case it would possibly give rise to Sn-based heterostructures with a protective layer. This, in principle, makes possible ambient condition measurements, or transport in ambient conditions between UHV systems. Since the $\mathrm{hBN}$ terminated $\mathrm{ZrB}_{2}$ surface is largely free of $\mathrm{Si}$, if ordered $\mathrm{Sn}$-structures form they may have distinctly different properties.

$\mathrm{h}-\mathrm{BN}$ is mentioned in chapter 3 as one of the suitable candidates for supporting stanene, one of a few with several theoretical papers but no experimental work reported. In case the deposited Sn stays on top of h-BN, the efforts need to be made to obtain stanene, by exploring parameter space (temperature, deposition rate, deposition temperature etc), possibly in similar direction as in the case of $\mathrm{MoS}_{2}$, as both materials are inert and interact very weakly with adlayers. 



\section{Summary}

Starting from the discovery of graphene, the two-dimensional (2D) materials field has received a massive increase in interests over the recent 15 years. Due to major obstacles in utilizing graphene for electronics, the attention of the scientific community turned to other elemental 2D materials, in particular from group IV of the periodic table. The elemental group IV 2D materials - among others - are theoretically predicted to exhibit very high carrier mobility and exotic topological properties, in particular the Quantum Spin Hall Effect.

Despite the promising theoretical results, experimental research on these materials struggles with the synthesis of material with properties that would match the theoretical predictions. The major obstacle is the lack of stability of the 2D lattice, related to the fact that the bulk crystalline structures do not favor any layered formations in these materials. Hence the most common way to stimulate layered growth is epitaxy. While the substrate supports the structural stability of a $2 \mathrm{D}$ material, the substrate-adlayer interactions alter and often completely remove the exotic (topological) properties from the 2D material. This tug-of-war relation makes finding a substrate that would stabilize the 2D material but at the same time interacts weakly a critical ingredient for the growth of 2D materials with desired properties. The goal to find such material was a primary motivation of this work.

In order to properly examine the aforementioned criteria of the epitaxial system a few complementary techniques were used. These are described in Chapter 1, which also contains a brief general introduction of the research scope. Scanning Tunneling Microscopy (STM) and Low Energy Electron Diffraction (LEED) provided information about structural ordering, while Scanning Tunneling Spectroscopy (STS), and (Angle Resolved) Photoelectron Spectroscopy ((AR)PES) gave insight into chemical composition and electronic structure of the obtained system.

In Chapter 2 we review and systematize the knowledge about materials that would be feasible substrates for the 2D material that is the main focus of this work - stanene, a Sn-based 2D material. The review contains information about the materials that have 
been theoretically predicted to support stanene, mainly by DFT calculations. We also highlight reports where stanene has been synthesized and summarize the properties of the material in each report.

Chapter 3 is a pioneer approach to combine two 2D materials in one system by growing epitaxial Sn on silicene terminated $\mathrm{ZrB} 2$. Out investigation showed that $\mathrm{Sn}$ grows on the substrate in various forms, in particular a mosaic-like alloy and an ordered monolayer. We did not, however, find any stanene layers. The insight into the electronic structure via STS shows a featureless gap in the DOS of the ordered layer. Our work is among the first on growing one elemental 2D material on top of the other. Successful growth of an alloy-like structure opens doors for 2D alloys, with yet to be determined properties. The discovery of various ordered $\mathrm{Sn}$ structures is a promising result in the direction of $2 \mathrm{D}$ heterostuctures research.

Chapter 4 shows our investigation on epitaxial growth of Sn on MoS2. A few works report on MoS2 being a feasible candidate for supporting stanene. The deposited Sn formed disordered, liquid-like droplets on the surface, which, upon annealing, etched the surface of the substrate. In order to obtain ordered Sn formations on the surface, we needed to decrease the deposition temperature of the sample. The obtained ordered islands of Sn are assigned to be composed of the $\beta$-Sn allotrope. Our results suggest that low-temperature deposition may stimulate the crystalline growth of Sn possibly in the low temperature allotrope $-\alpha-\mathrm{Sn}$, a monolayer of which corresponds to stanene.

Finally, in Chapter 5 we investigate the dependence of $\mathrm{Li}$ intercalation between multilayer graphene sheets on the defect density in the graphene. The defects were imposed in a controlled way in multilayer graphene on $\mathrm{Ni}(111)$ substrates and varied over a few orders of magnitude. Counterintuitively, the number of defects did not influence the intercalation capacity. The reason for this was attributed to step edges taking the primary role in the diffusion of $\mathrm{Li}$ in between graphene sheets, as well as a clogging mechanism, where Li atoms accumulate on the defects, effectively screening them from other atoms. 


\section{Samenvatting}

Sinds de ontdekking van grafeen heeft het onderzoeksveld dat tweedimensionale (2D) materialen bestudeerd de afgelopen 15 jaar een enorme toename van de belangstelling gekregen. Vanwege grote obstakels bij het gebruik van grafeen voor elektronica, richtte de aandacht van de wetenschappelijke gemeenschap zich op andere elementaire 2Dmaterialen, met name uit groep IV van het periodiek systeem. Theoretisch is voorspeld dat, onder andere, de elementaire groep IV 2D-materialen een zeer hoge mobiliteit van ladingsdragers en exotische topologische eigenschappen vertonen, met name het Quantum Spin Hall-effect.

Ondanks de veelbelovende theoretische resultaten, worstelt experimenteel onderzoek naar deze materialen met de synthese van een materiaal met eigenschappen die overeenkomen met de theoretische voorspellingen. Het grootste obstakel is het gebrek aan stabiliteit van het 2D-rooster, gerelateerd aan het feit dat de bulk kristallijne structuren geen gelaagde formaties in deze materialen vertonen. Daarom is epitaxie de meest gebruikelijke manier om gelaagde groei te stimuleren. Terwijl de substraatondersteuning helpt bij het vergroten van de structurele stabiliteit van een 2Dmateriaal, veranderen de substraat-adlayer-interacties de eigenschappen, en verwijderen ze vaak de exotische (topologische) eigenschappen van het 2D-materiaal volledig. Deze touwtrekrelatie maakt het vinden van een substraat dat het 2D-materiaal zou stabiliseren, maar tegelijkertijd zwakke interactie vertoond een cruciaal ingrediënt voor de groei van 2D-materialen met de gewenste eigenschappen. Het doel om een dergelijk materiaal te vinden was één van de belangrijkste motivaties van dit werk.

Om de bovengenoemde criteria van het epitaxiale systeem goed te onderzoeken, werden enkele complementaire technieken gebruikt. Deze worden beschreven in hoofdstuk 1, waarin ook een korte algemene introductie van het onderzoeksgebied is opgenomen. Scanning Tunneling Microscopy (STM) en Low Energy Electron Diffraction (LEED) verschaften informatie over structurele ordening, terwijl Scanning Tunneling Spectroscopy (STS) en (Angle Resolved) Photoelectron spectroscopy Spectroscopy ((AR) PES) inzicht gaven in chemische samenstelling en elektronische structuur van het verkregen systeem. 
In hoofdstuk 2 geven we een overzicht van de kennis over materialen die geschikte substraten zouden kunnen zijn voor de 2D-materialen en systematiseren we de deze, met name voor het materiaal dat de belangrijkste focus is van dit werk - Staneen, een op Sn gebaseerd 2D-materiaal. Het overzicht bevat informatie over de materialen waarvan theoretisch is voorspeld dat ze stanene ondersteunen, voornamelijk door middel van DFT-berekeningen. We benadrukken ook rapporten waarin staneen is gesynthetiseerd en vatten de eigenschappen van het materiaal in elk rapport samen.

Hoofdstuk 3 is een pioniersbenadering om twee 2D-materialen in één systeem te combineren door het groeien van epitaxiaal Sn op met siliceen getermineerd $\mathrm{ZrB} 2$. Uit ons onderzoek is gebleken dat $\mathrm{Sn}$ in verschillende vormen op het substraat groeit, met name een mozaïekachtige legering en een geordende monolaag. We hebben echter geen staneenlagen gevonden. Het inzicht in de elektronenstructuur via STS laat een gap zonder overige opvallende kenmerken zien in de DOS van de geordende laag. Ons werk is een van de eersten dat de groei van het ene elementaire 2D-materiaal bovenop het andere laat zien. Succesvolle groei van een legeringsachtige structuur opent deuren voor 2D-legeringen, met nog verder te bepalen eigenschappen. De ontdekking van verschillende geordende Sn-structuren is een veelbelovend resultaat voor de ontwikkeling van 2D-heterostucturen.

Hoofdstuk 4 rapporteert ons onderzoek naar epitaxiale groei van Sn op MoS2. Een paar studies melden dat MoS2 een geschikte kandidaat is als substraat om stanene te ondersteunen. Het gedopeerde Sn vormde ongeordende, vloeistofachtige druppels op het oppervlak, die bij verhitten het oppervlak van het substraat etsten. Om een geordende Sn-formatie op het oppervlak te verkrijgen, moesten we de depositietemperatuur van het sample verlagen. De verkregen geordende eilanden van Sn worden toegeschreven te zijn samengesteld uit de $\beta$-Sn-allotroop. Onze resultaten suggereren dat depositie bij lagere temperatuur de kristallijne groei van de $\alpha$-Sn allotroop mogelijk zou kunnen plaatsvinden, waarvan een monolaag overeenkomt met staneen.

Tenslotte onderzoeken we in hoofdstuk 5 de afhankelijkheid van Li-intercalatie in multilayer grafeen van de dichtheid van defecten in het grafeen. De defecten werden gecontroleerd aangebracht in multilayer grafeen op $\mathrm{Ni}$ (111) -substraten, en de defectdichtheid werd gevarieerd over een paar ordes van grootte. Contra-intuïtief had het aantal defecten geen invloed op de intercalatiecapaciteit. De reden hiervoor werd toegeschreven aan het feit dat stapranden de primaire rol spelen in de diffusie van $\mathrm{Li}$ 
tussen individuele grafeenlagen, evenals een verstoppingsmechanisme, waarbij Liatomen zich ophopen op de defecten, waardoor ze effectief worden afgeschermd van andere atomen. 



\section{List of publications}

M. Ochapski, A. Fleurence, J. Gou, P. Markeev, P.K.J. Wong, Y. Yamada - Takamura, A.T.S. Wee, M.P. de Jong, Epitaxial monolayer of $\mathrm{Sn}$ on silicene teminated $\mathrm{ZrB}_{2}$ (manuscript in preparation)

M. Ochapski, A. Fleurence, J. Gou, P. Markeev ,P.K.J. Wong, Y. Yamada - Takamura, A.T.S. Wee, M.P. de Jong, Epitaxial $2 D$ honeycomb $S n_{x} S i_{1-x}$ alloy (manuscript in preparation)

M. Ochapski, A. Fleurence, J. Gou, P. Markeev, P.K.J. Wong, Y. Yamada - Takamura, A.T.S. Wee, M.P. de Jong, Formation of a metallic silicon bilayer on silicene/ZrB ${ }_{2}$ by Sn deposition (manuscript in preparation)

M. Ochapski, M.P. de Jong, Substrates feasible for epitaxial growth of stanene - review (manuscript in preparation)

M. Ochapski, D. Ataç, J.G.M. Sanderink, Alexey Y. Kovalgin, M.P. de Jong, Li intercalation into multilayer graphene with controlled defect densities (manuscript submitted)

M. Ochapski, E. Urbain, F. Djeghloul, V. Speisser, H. Majjad, D. Spor, A. D. Vu, J. Coraux, N. Rougemaille, G. Chen, A. K. Schmid, M. Suzuki, T. Yasue, T. Koshikawa, $\mathrm{H}$. Bulou, and W. Weber, Breakdown of the electron-spin motion upon reflection at metal-organic or metal-carbon interfaces. II., Phys. Rev. B, 93, 17, 174411 (2016) 



\section{Acknowledgements}

After 4 years my PhD journey ends. The outcome, in the form of this book but not only, would not be possible without many people whose scientific and non-scientific help and support accompanied me in this enterprise.

First and foremost, the one and only, Prof. Michel de Jong. I'm very grateful for giving me a chance to do the $\mathrm{PhD}$ under your supervision. It's hard to express how much I appreciate your guidance, given not only by always useful advice, but also by invaluable example of scientific work ethos, full of curiosity, motivation and excitement shown in action. You are my role model of what a scientist should be and of what scientific approach should look like. I very much appreciate all the support you gave me during my $\mathrm{PhD}$ track.

I would also like to thank Prof. Harold Zandvliet for being my co-promotor. Thank you for giving me an opportunity to cooperate and exchange thoughts with you and your students.

Many thanks also to Prof. Wilfred van der Wiel. Thank you for the opportunity to work in your group.

Collaborations played an important role in my $\mathrm{PhD}$ and it was a great pleasure to work together with Prof. Yukiko Yamada-Takamura and Dr. Antoine Fleurence from Japan Advanced Institute of Science and Technology. Yukiko-sensei thank you for the opportunity to visit your group, it was a very fruitful time and an amazing experience. Antoine, your help and advice during my time in JAIST, but also on multiple joint synchrotron beam times was invaluable. I learnt a lot from you and enjoyed the goods and the bads of beam times together.

Then I want to thank Prof. Andrew Wee and Prof. Johnny Wong from National University of Singapore for another fruitful collaboration. Thank you for making it possible for me to visit your group and for organizing my stay. I would also like to thank Dr. Gou Jian for being my daily supervisor at NUS, and for sharing his knowledge and experience with me. 
Furthermore, I want to thank Prof. Koster and Dr. Kovalgin and Prof. Banerjee for being in my committee. Thank you for your time and effort. I believe your comments and remarks increased the quality of my work thesis.

During my defense I will be assisted by my two paranymphs: Yigitcan Uzun and Robert Ukropec. Boys, we started this together and we finish together. I'm very happy and grateful to have you by my side as I have had for the last 4 years.

Yigitcan, we shared office for 4 long years. It still feels like it wasn't that long ago. It was a great experience to start every day with a little chat with you. It was so much fun to live our short snowboard life together. Still remember all the trips we did together to many places in Europe and our random meetings on trips we planned separately! Thanks to you I know how delicious Turkish food is, and how to ask for discount in a kebab shop. Thank you for being a reliable and supportive friend in times I needed it. And of course, can't forget about all the football we played together!

Robert, it's an honor to be your friend. Your optimism and charisma always lightened up the room, at many parties, trips and other events we shared together. One can never be bored with you. Apart from a party persona I also really value your intellectual side which you showed to me during our chats. I admire the way you conduct a discussion and dialogue, with vast knowledge and insightful remarks. You made me question many things and revaluate my views and their foundation. Thank you for all the inspiration! I also enjoyed a lot our struggle together in the last months (especially Wednesday burgers!). I should also not forget our gaming sessions, especially Civ6, during which I was constantly trembling in fear for my life. I will not forget that and I promise you revenge.

Then the other staff members deserve a word. I would like to thank Karen and Judith for dealing with all the paperwork a traveling $\mathrm{PhD}$ can generate. You always had time and were happy to help. Thank you for that.

Floris, I'm very happy to have met you and to have shared everyday office life with you. Thank you for your support and help in organizing group meetings and support you showed every time I needed it. Finally thank you for all the football we played together, you're irreplaceable in NanoElectronics team!

Peter, I appreciate your insightful questions on group meetings and your readiness to help and advice. 
Next, I would like to thank technicians, Johnny and Martin. Thanks a lot for keeping things working with your "technician aura". Your help was invaluable and I'm very grateful to have a chance to work with you.

I would also like to thank Tao and Alejandro for being my officemates. Tao I enjoyed our chats, I always listened with interest your stories. You're also an example for me in your successful efforts to publish your paper in Nature. Alejandro it was good to know you and to share office. I will remember our long chats and my first shy attempts in calisthenics. I hope you've found your happiness.

Unai, I will not forget our sailing trip and football, as well as cinema trips and long chats. I'm very happy to have met you.

I also need to thank Pavel. Thanks for all the laughs and memes, the shooting range and bike trips! Also, thank you for hard work at synchrotrons and in the lab.

Furthermore, I would like to thank all the other group members. Derya, Guus, Antonio, Bram, Hans-Christian, Zhen, Dilu, Oguzhan, Gabi and others who made my days in NanoElectronics an awesome experience. We played football, games, went on trips and spare-ribs Mondays. Thank you for being excellent colleagues and for making my days in NanoElectronics an amazing experience.

Then there are few people from outside of the group that I would like to acknowledge. I'm grateful to the members of PIN group for being welcoming and supportive professionally, as well as a great company socially. Special thanks to Kai, whose enthusiasm and advice were an inspiration to me.

Then, the friends I met and who made my past-time fun. It's not possible to mention everyone by name, but I will try nonetheless. Nga, thank you for being a great friend, always happy with both intellectual discussions and memes. I enjoyed time we spent on many activities and trips.

Weronika, Shofiyati, Ha, Haruka, Takuma, Enrico, Omran. Thank you for shared moments and great memories we made together.

I was also very lucky to meet you, Shasha. You have brightened up the hard part of my $\mathrm{PhD}$ with your optimism, always-smiling face and unconditional support. Thanks to you I made many unforgettable memories and enjoyed every single one of them. 
Finally, I would like to thank my parents, Jolanta and Witold and my brothers, Filip and Bartosz, who were always there to give support and encouragement. Now I can, finally, proudly say: Grandma, I have finished school! 